\title{
NUMERICAL INVESTIGATION OF THE IMPINGEMENT OF A PLANAR JET OF NANOFLUIDS ON A V-SHAPED PLATE
}

\author{
by \\ Nishma Bhatt \\ A project \\ presented to Ryerson University \\ in partial fulfilment of the \\ requirements for the degree of \\ Master of Engineering \\ in the program of \\ Aerospace Engineering
}

Bachelor of Engineering, Gujarat Technological University (2014)

Toronto, Ontario, Canada, 2017

CNishma Bhatt 2017 


\section{AUTHOR'S DECLARATION FOR ELECTRONIC SUBMISSION OF A PROJECT}

I hereby declare that I am the sole author of this project. This is a true copy of the project, including any required final revisions, as accepted by my examiners.

I authorize Ryerson University to lend this project to other institutions or individuals for the purpose of scholarly research.

I further authorize Ryerson University to reproduce this project by photocopying or by other means, in total or in part, at the request of other institutions or individuals for the purpose of scholarly research.

I understand that my project may be made electronically available to the public. 


\title{
NUMERICAL INVESTIGATION OF THE IMPINGEMENT OF A PLANAR JET OF NANOFLUIDS ON A V-SHAPED PLATE
}

\author{
Nishma Bhatt \\ Master of Engineering, Aerospace Engineering, Ryerson University, Toronto (2017)
}

\begin{abstract}
An effective way to enhance the heat dissipation in industrial heat transfer devices is impinging of the fluid jet. Due to the higher dissipation heat flux, jet flows can be used for to control the temperature of high intensity heat sources.

Traditional fluids such as water, ethylene and propylene glycol, and oils offer heat transfer capabilities that are adequate for many applications. There are several options to increase the effectiveness of the heat transfer characteristics for these fluids, for instance, using jet flows, and increasing the surface area of the heat transfer object. However, with the advances in nanotechnology and material science, nanofluids offer an attractive alternative option. Nanofluids refer to a dispersion of metallic or non-metallic particles with dimensions smaller than $100 \mathrm{~nm}$ in a base fluid like water, ethylene and propylene glycol, oil. Nanofluids have been shown to have an enhanced heat transfer characteristic, because of their high thermal conductivity.

In this Project, Heat transfer enhancement of an impinging liquid jet on a V-shape target plate cooling system, has been investigated numerically, by replacing the base fluid, water, with $\mathrm{Al}_{2} \mathrm{O}_{3}$-water nanofluid. To conduct the research, literature review on nanofluid heat transfer enhancement, jet impingement, and nanofluids jet impingement, has been conducted. Numerical model has been built using ANSYS Workbench 16.0. After validating the numerical code with the previous experimental data, the effect of nanoparticles volume fraction, jet-surface distance and jet's Reynolds number on the heat transfer enhancement has been investigated.
\end{abstract}




\section{ACKNOWLEDGEMENT}

Dr. Tooraj Yousefi

Dr. Bo Tan 


\section{TABLE OF CONTENTS}

\begin{tabular}{|c|c|c|}
\hline Chapter & Title & Page \# \\
\hline & $\begin{array}{l}\text { Author's Declaration } \\
\text { List of Tables } \\
\text { List of Figures }\end{array}$ & $\begin{array}{l}\text { ii } \\
\text { vi } \\
\text { vii }\end{array}$ \\
\hline 1 & $\begin{array}{l}\text { 1.1 Introduction } \\
\text { 1.2 Literature Survey } \\
\text { 1.3 Objective }\end{array}$ & $\begin{array}{l}1 \\
2 \\
4\end{array}$ \\
\hline 2 & $\begin{array}{l}\text { 2.1 Introduction to Jet Fluid } \\
\text { 2.1.1 Laminar Flow } \\
\text { 2.1.2 Boundary Layer: Thermal and Velocity } \\
\text { 2.1.3 Heat Transfer Coefficient Relation with Reynolds number } \\
\text { 2.2 Introduction to Nanofluids } \\
\text { 2.2.1 Synthesis of Nanofluids } \\
\text { 2.2.2 Thermal Conductivity of Oxide Nanofluids } \\
\text { 2.2.3 Convection in Nanofluids }\end{array}$ & $\begin{array}{l}5 \\
5 \\
6 \\
9 \\
10 \\
11 \\
11 \\
16\end{array}$ \\
\hline 3 & $\begin{array}{l}\text { 3.1 Numerical Model Background } \\
\text { 3.1.1 k- } \varepsilon \text { model for Mixed Regime Jet flow } \\
\text { 3.2 Computational domain and Mesh }\end{array}$ & $\begin{array}{l}18 \\
20 \\
21\end{array}$ \\
\hline 4 & $\begin{array}{l}\text { 4.1 Results } \\
\text { 4.1.1 Plate temperature for Water \& Nanofluid } \\
\text { 4.1.2 Local heat transfer coefficients of water } \\
\text { 4.1.3 Local heat transfer coefficients of Al2O3-water nanofluid } \\
\text { 4.1.4 Average heat transfer coefficients of Al2O3-water nanofluid } \\
\text { 4.2 Conclusion }\end{array}$ & $\begin{array}{l}26 \\
26 \\
29 \\
30 \\
35 \\
36\end{array}$ \\
\hline & APPENDIX A & 38 \\
\hline & APPENDIX B & 45 \\
\hline & REFERENCES & 48 \\
\hline
\end{tabular}




\section{LIST OF TABLES}

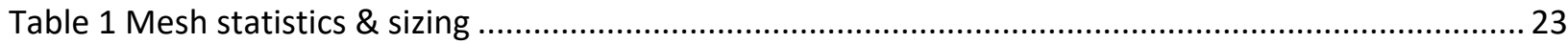

Table 2 Mesh precision: Mesh Dependency Study for case Nanofluid $\mathrm{Al}_{2} \mathrm{O}_{3}-\mathrm{H} 2 \mathrm{O}(0.05 \%$ \& All Re) .......... 23

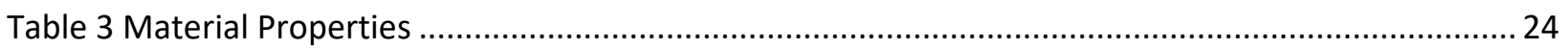

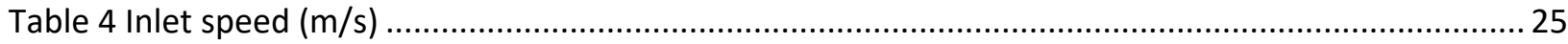




\section{LIST OF FIGURES}

Figure 1 (a) The experimental setup (b) schematic diagram of planar jet impinging on the target plate [9]

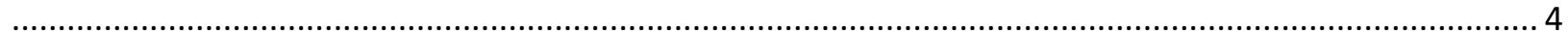

Figure 2 Growth of a boundary layer on a flat plate

Figure 3 Velocity boundary layer growth $(\delta)$ that starts from the leading edge of the plate. The thermal boundary layer $\left(\delta_{t}\right)$ starts after a distance $(\xi)$ from where the temperature of the plate changes from ambient temperature to a different temperature $\left(T_{s}\right)$, causing convection heat transfer........................9

Figure 4 Heat Transfer Coefficient on a Flat plate for different flow regime ......................................... 10

Figure 5 Enhanced thermal conductivity of Oxides nanofluids system [17].........................................12

Figure 6 Comparison of $\mathrm{Al}_{2} \mathrm{O}_{3}$-Water nanofluid conductivity using Hamilton-Crosser theory [18] ........... 13

Figure 7 Comparison of $\mathrm{Al}_{2} \mathrm{O}_{3}$-Ehylene glycol nanofluid conductivity using Hamilton-Crosser theory [18]

Figure 8 Comparison of CuO-Water nanofluid conductivity using Hamilton-Crosser theory [18] ............ 14

Figure 9 Comparison of CuO-Ethylene glycol nanofluid conductivity using Hamilton-Crosser theory [18]

Figure 10 comparison of $\mathrm{Al}_{2} \mathrm{O}_{3}$-Water Nanofluid conductivity from Lee-Choi \& Masuda experiments

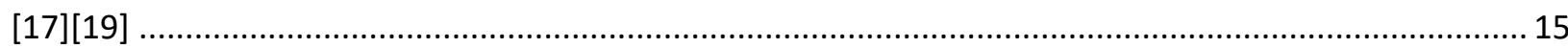

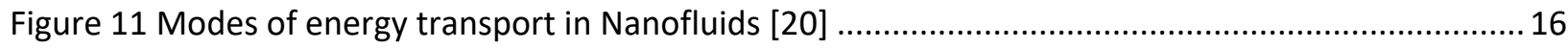

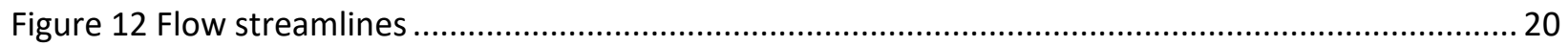

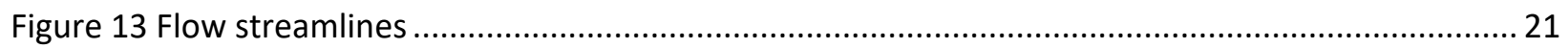

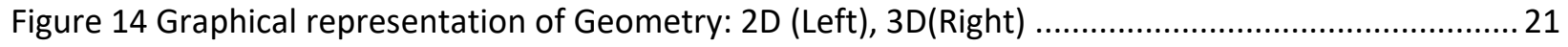

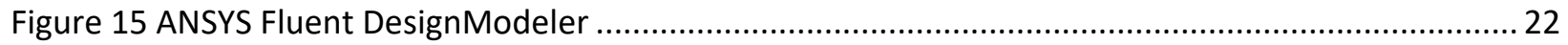

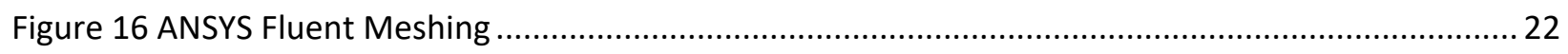

Figure 17 Plate temperature distribution of the one side of the target plate for water..........................26

Figure 18 Plate temperature distribution of the one side of the target plate for $\mathrm{Al}_{2} \mathrm{O}_{3}$-water nanofluid

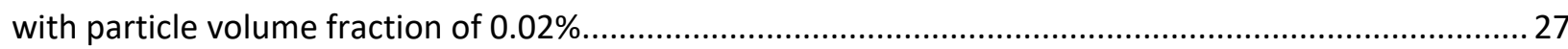

Figure 19 Plate temperature distribution of the one side of the target plate for $\mathrm{Al}_{2} \mathrm{O}_{3}$-water nanofluid

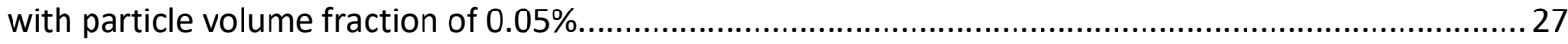

Figure 20 Plate temperature distribution of the one side of the target plate for $\mathrm{Al}_{2} \mathrm{O}_{3}$-water nanofluid

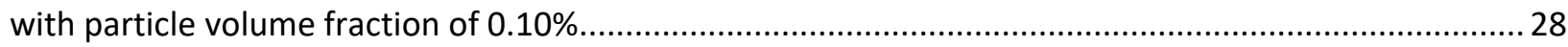

Figure 21 Plate temperature distribution of the one side of the target plate for $\mathrm{Al}_{2} \mathrm{O}_{3}$-water nanofluid

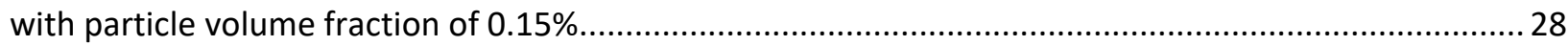

Figure 22 ANSYS CFD Post Surface heat transfer coefficient output: Water at Re 2719 ........................29

Figure 23 Distribution of the local impinging jet heat transfer coefficient with the use of water.............30

Figure 24 ANSYS CFD Post Surface heat transfer coefficient output: Nanofluid $0.05 \%$ at Re $2719 \ldots \ldots \ldots . . .31$

Figure 25 Distribution of the local impinging jet heat transfer coefficient with the use of $\mathrm{Al}_{2} \mathrm{O}_{3}-$ water

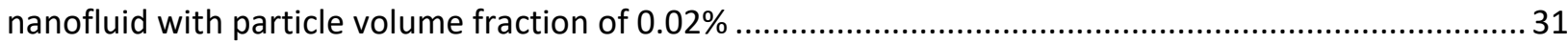

Figure 26 Distribution of the local impinging jet heat transfer coefficient with the use of $\mathrm{Al}_{2} \mathrm{O}_{3}$-water nanofluid with particle volume fraction of $0.05 \%$.

Figure 27 Distribution of the local impinging jet heat transfer coefficient with the use of $\mathrm{Al}_{2} \mathrm{O}_{3}-$ water nanofluid with particle volume fraction of $0.10 \%$ 
Figure 28 Distribution of the local impinging jet heat transfer coefficient with the use of $\mathrm{Al}_{2} \mathrm{O}_{3}-$ water

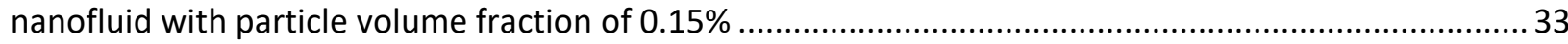

Figure 29 Local heat transfer coefficients versus nanoparticle volume concentrations at Re $1732 \ldots . . . . . .33$

Figure 30 Local heat transfer coefficients versus nanoparticle volume concentrations at Re $2000 \ldots . . . . . .33$

Figure 31 Local heat transfer coefficients versus nanoparticle volume concentrations at Re $2261 \ldots \ldots . . . .34$

Figure 32 Local heat transfer coefficients versus nanoparticle volume concentrations at Re 2500 .......... 34

Figure 33 Local heat transfer coefficients versus nanoparticle volume concentrations at Re $2719 \ldots \ldots . . .34$

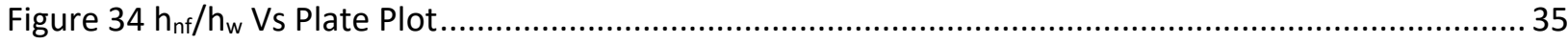

Figure 35 Average Surface heat transfer coefficient for plate ( $h$ num ) Plot w.r.t Reynolds number ....... 36 


\section{CHAPTER 1}

\subsection{Introduction}

Ultra-high cooling is highly vital for industrial technologies. However, inherently low thermal conductivity is a primary limiting factor, responsible for the poor cooling effect. One of the cooling methods is the jet impingement of a liquid or gas onto a surface on a continuous basis. This mode of cooling \& heat transfer has been tested extensively for many years and is advancing every day.

Conventional Impingement Jets can either be air-powered or use some form of a liquid, typically water. High-speed jet impingement on a component surface creates a thin boundary layer, and thus a high heat transfer. Mainly, there are three jet configurations: the free-surface jet, which uses a dense liquid in a medium that is less dense, such as air; the submerged jet, which allows the fluid to impinge in the same medium fluid; and the confined submerged jet, if there are walls nearby, this will limit the motion of the fluid.

A liquid jet is a stream of fluid having somewhat columnar shape. It serves a large variety of situations, spanning a broad range of physical length scales, hence the wide scope of this review. A Liquid jet is a stream projection of fluid into a surrounding medium, usually from a nozzle, aperture or orifice. Liquid Jets can travel long distances without major dissipation. The momentum of Jet fluid is higher, compared to the surrounding fluid medium. if the surrounding medium is the same fluid as the jet fluid, and this fluid has a viscosity, the surrounding fluid is carried along with the jet in a process called entrainment. Stagnation point flow represents a fluid flow in the immediate neighborhood of solid surface at which fluid approaching the surface divides into different streams.

In the development of energy efficient heat transfer fluids, the thermal conductivity of the heat transfer fluids plays a vital role. Modern nanotechnology has enabled the production of metallic or non-metallic nanoparticles with average crystallite sizes below 100nm. The mechanical, optical, electrical, magnetic, and thermal properties are superior to conventional materials. Recognizing an excellent opportunity to apply nanotechnology to thermal engineering, nanofluids are used with combination of other fluids, especially as a cooling add-on. 


\subsection{Literature Survey}

Fluid Jets are very useful for surface cooling because of high efficiency and simple mechanism. Lots of studies and research work has been conducted on Fluid jet with nanofluid.

Analytical and Experimental Studies have been conducted for the derivation of complete expressions for radial distribution of the local Nusselt number under an impinging jet [1]. Studies on Flow properties such as turbulent, laminar and splattering jets have also been conducted. Boundary layer at stagnation point are very thin, this results in very high heat transfer coefficients, occasionally exceeding $100 \mathrm{~kW} / \mathrm{m}^{2} \mathrm{~K}[2]$.

In consequence, wall roughness and conjugate heat transfer effects can also have a nontrivial influence [3]. The fluid film forms on the target surface, due to the shower of droplets expelled by liquid jets, when impinging on a solid surface. This layer lowers the cooling efficiency or reduced coating efficiency. It has been analyzed that splattering is driven by disturbances on the surface of the impinging jet. Due to liquid-side pressure fluctuations, turbulent jets develop roughness on the surface. So, turbulent jets, turbulence is the usual cause of splattering in nonboiling systems [4].

In another experiment, for impingement of nanofluid via a slot jet, it was justified that, 0.0597 wt\% $\mathrm{YAl}_{2} \mathrm{O}_{3}-\mathrm{H}_{2} \mathrm{O}$ nanofluid is most optimum concentration, for maximum heat transfer at a range of Reynold numbers [5]. Large-Eddy simulation and experiments have been conducted to characterize the predictions of the flow from the numerical simulations to control the heat transfer on the impinging wall.

When impinging of a circular jet is considered, heat transfer can be divided into two different regions, a consequence of the development of the viscous and thermal boundary layers and the possible occurrence of hydraulic jump or a nucleate boiling. These regions show varied trends according to the jet Reynolds number, temperature level, fluid physical properties, and wall heat flux. The Nusselt number varies radially, and these variations have direct relation with the development of thermal boundary layer \& research has presented an integral analysis of each convective regions [6]. In one research Nanofluid correlation with heat transfer was studied, according to which, nanofluid suspended nanoparticles are responsible for a change in heat transfer performance and transport characteristics of nanofluid, promoting high thermal conductivity. Due to ultra-fine particles, nanofluid behaves more like a single phase fluid [7]. In water-Nanoparticle mixture, addition of small 
concentration of particles enhances the thermal conductivity of the base fluid, and that there is an additional enhancement of the convective heat transfer coefficient in laminar flows. Also, it appears that there is an enhancement of the CHF, while the boiling heat transfer coefficient typically decreases by a small amount [8][9]. The experiment was conducted to study heat transfer and viscous pressure loss properties of alumina-water nanofluid, in a flow loop with a vertical heated tube, in laminar flow regime. Results showed that $6 \mathrm{vol} \%$ alumina nanofluid heat transfer coefficient was $27 \%$ higher than that of water in the entrance region. This suggests that the nanofluids behave as homogeneous mixtures. Pressure loss for the nanofluid is higher than water's but varies linearly with the fluid viscosity, similar to the conventional pressure loss theory for laminar flow [10]. Pressure drop and convective heat transfer coefficient have been measured, for water- $\mathrm{Al}_{2} \mathrm{O}_{3}$ nanofluid flowing through a uniformly heated circular tube in the fully developed laminar flow regime. The nanofluid experimental friction factor agreed with analytical predictions from Darcy's equation for single-phase flow. At a concentration of $0.3 \mathrm{vol} \%$ heat transfer coefficient increased by $8 \%$, compared to pure water. Furthermore, it was proposed that flattening of the velocity profile, induced from large gradients in bulk properties such as nanoparticle concentration, thermal conductivity, and viscosity, is a possible mechanism for the convective heat transfer coefficient enhancement exceeding the thermal conductivity enhancement [11].

Direct numerical simulation of heavy particles in three-dimensional well-developed turbulent shear flows, of a two-phase three-dimensional planar jet was investigated. When the flow was well developed, the particle dispersion \& particle dynamics were non-linear and dependent on the particle Stokes number, from the statistical point of view. It was demonstrated that the Stokes number had a notable effect on interparticle relative velocity, ensemble-averaged slip velocity, and ensemble-averaged particle kinetic energy [12]. Numerical study was performed to simulate the forced convection flow of $\mathrm{Al}_{2} \mathrm{O}_{3}$-water nanofluid in the radial flow cooling system using a single-phase approach. Outcome show the heat transfer coefficient increases with the increase of the nanoparticle volume fraction and the Reynolds number. On the adverse side, the increase in pressure drop was directly related with the increase of particle concentration. Considering both, the cooling effect \& pressure drop, no better heat transfer enhancement is found with the use of nanofluid compared to that of pure water under the laminar, medium-heat flux conditions in the radial flow system [13]. 


\subsection{Objective}

The experiment was conducted by Dr. Tooraj Yousefi and his team members. According to that experiment, a jet of $\mathrm{Al}_{2} \mathrm{O}_{3}$-water nanofluid, at volume fractions $(0.02 \%, 0.05 \%, 0.1 \%$, and $0.15 \%)$, was used to impinge vertically on the vertex of a $\mathrm{V}$-shaped plate. Under laminar flow regime, heat transfer coefficient of the nanofluid jet was measured, for all the cases, and compared with the pure water readings. Variation in Reynolds numbers was also implemented, ranging from 1732 to 2719. End results of the experiment show that both local and average heat transfer coefficients were at best optimum value, for $\mathrm{Al}_{2} \mathrm{O}_{3}$-water nanofluid at low volume fractions of $0.02 \%$ and $0.05 \%$, compared to all other cases. for Reynolds number less than 2250, local \& average heat transfer coefficient has been decreased with increasing Reynolds number. The trend was reversed for the Reynolds number greater than 2250 [9].

In this study, Numerical Investigation, on the Impingement of a planar jet of nanofluids on a V-shaped plate, has been conducted. The heat transfer enhancement of an impinging jet on a $\mathrm{V}$-shape target plate has been investigated by replacing the base fluid, water, with $\mathrm{Al}_{2} \mathrm{O}_{3}$-Water nanofluid. Numerical model has been built using Ansys Workbench 16.0. After validating the numerical results with the previous experimental data, the effect of nanoparticles volume fraction, jet-surface distance and jet's Reynolds number on the heat transfer enhancement has been investigated.

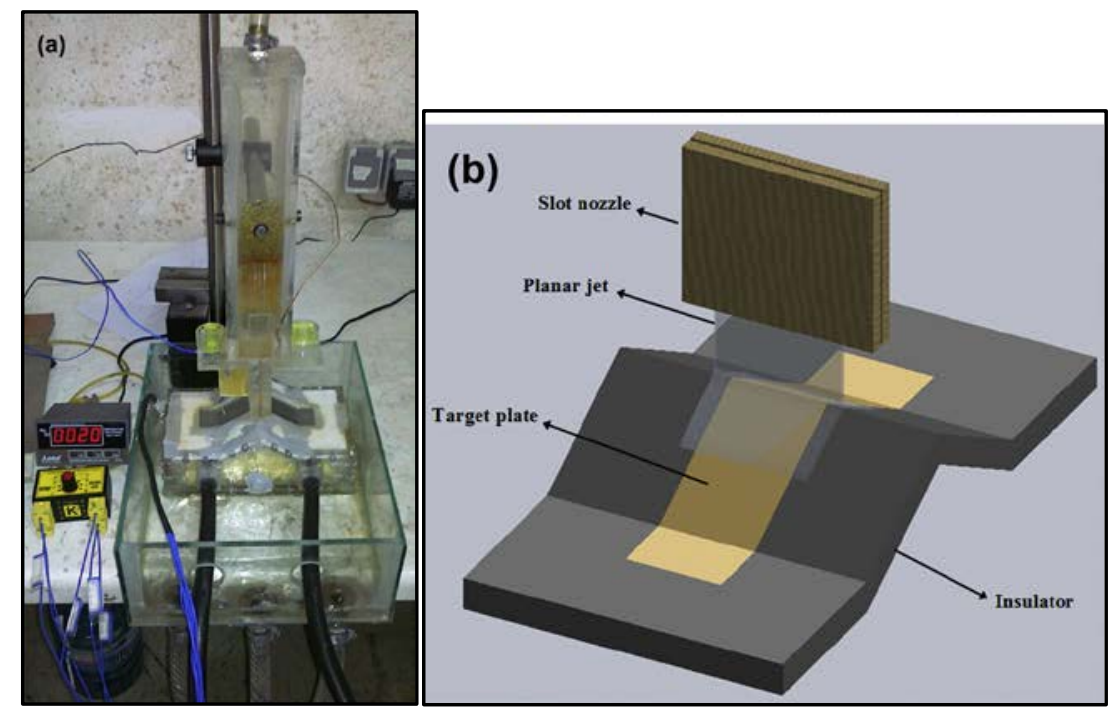

Figure 1 (a) The experimental setup (b) schematic diagram of planar jet impinging on the target plate [9] 


\section{CHAPTER 2}

\subsection{Introduction to Jet Fluid}

A jet is a stream of fluid that is projected into a surrounding medium, usually from nozzle, aperture or orifice. Jets can travel long distances without dissipating. Jet fluid has higher momentum compared to the surrounding fluid medium. In the case that the surrounding medium is assumed to be made up of the same fluid as the jet, and this fluid has a viscosity, the surrounding fluid is carried along with the jet in a process called entrainment. For the current study, surrounding medium is air, so entrainment can be ignored. Also, flow will be under the laminar flow regime.

\subsubsection{Laminar \& Turbulent Flow}

When fluid flows with no disruption between layers i.e. in parallel layers, it is known as laminar flow. The fluid flows without lateral mixing and adjacent layers slide past one another in parallel order, at low velocity. There are no cross-currents perpendicular to the direction of flow, nor eddies or swirls of fluids. Laminar flow is a flow regime characterized by high momentum diffusion and low momentum convection.

The type of flow decides heat and mass transfer properties in fluid systems. The dimensionless Reynolds number is an important parameter in the equations that describe whether fully developed flow conditions lead to laminar or turbulent flow. The Reynolds number is the ratio of the inertial force to the shearing force of the fluid-how fast the fluid is moving relative to how viscous the fluid is, irrespective of the scale of the fluid system. Laminar flow generally occurs when the fluid is moving slowly or the fluid is very viscous. As the Reynolds number increases, such as by increasing the flow rate of the fluid, the flow will transition from laminar to turbulent flow at a specific range of Reynolds numbers, the laminar-turbulent transition range depending on small disturbance levels in the fluid or imperfections in the flow system. For the current study, range of Reynolds number is 1732-2719.

$$
R e=\frac{\rho U D}{\mu}
$$

where, $\mathrm{U}=$ fluid velocity, $\mathrm{D}=$ width of planar jet at the impingement, $\rho=$ Fluid Density \& $\mu=$ Fluid Viscosity 
For flat plate systems, laminar flow occurs when the Reynolds number is below a critical value of approximately $5 \times 10^{5}$, and for circular tube the transition range is typically 2,300 .

\subsubsection{Boundary Layer: Thermal and Velocity}

A boundary layer is a thin layer of viscous fluid close to the solid surface of a wall in contact with a moving stream in which (within its thickness $\delta$ ) the flow velocity varies from zero at the wall (where the flow "sticks" to the wall because of its viscosity) up to $U_{e}$ at the boundary, which approximately (within $1 \%$ error) corresponds to the free stream velocity.

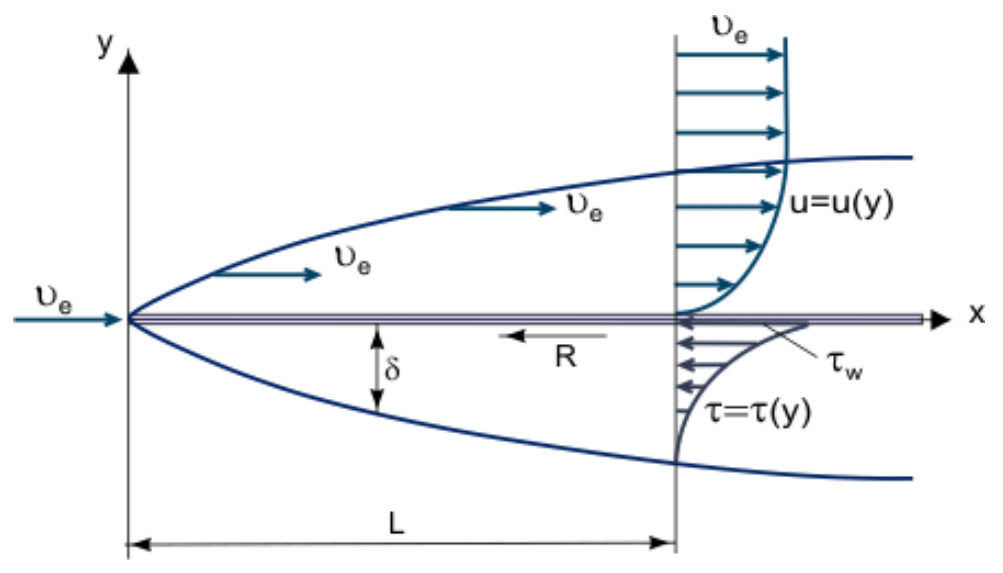

Figure 2 Growth of a boundary layer on a flat plate

Boundary layer can be defined as a layer of fluid developing in flows with very high Reynolds Numbers Re, that is with relatively low viscosity as compared with inertia forces. The order of magnitude of the boundary layer thickness can be estimated from the following relationship:

$$
\frac{\delta}{L}=R e^{-0.5}
$$

Gradually, under the influence of some destabilizing factors, the boundary layer becomes unstable and transition of boundary layer to a Turbulent Flow regime takes place. In some cases, the boundary layer becomes turbulent immediately downstream of the stagnation point of the flow. The boundary layer determines the aerodynamic drag and lift of the flying vehicle, or the energy loss for fluid flow in channels (in this case, a hydrodynamic boundary layer because there is also a thermal boundary layer which determines the thermodynamic interaction of Heat Transfer). Computation of the boundary layer parameters is based on the solution of equations obtained from the Navier-Stokes equations for viscous fluid motion. A 
transformation of the Navier-Stokes equation into the boundary layer equations can be demonstrated by deriving the Prandtl equation for laminar boundary layer in a two-dimensional incompressible flow without body forces. In this case, ignoring small terms the system of Prandtl equations for laminar boundary layer becomes:

$$
\begin{gathered}
\frac{\partial u}{\partial t}+u \frac{\partial u}{\partial x}+v \frac{\partial u}{\partial y}=\frac{-1}{\rho} \frac{\partial p}{\partial x}+v\left(\frac{\partial^{2} u}{\partial x^{2}}+\frac{\partial^{2} u}{\partial y^{2}}\right) \\
\frac{\partial u}{\partial x}+\frac{\partial v}{\partial y}=0
\end{gathered}
$$

The longitudinal pressure gradient $\left[\frac{\partial p}{\partial x}\right]=\left[\frac{d p}{d x}\right]$ (at $\mathrm{p}(\mathrm{y})=$ constant) in Eq. (3) can be depicted from the Euler equation of motion of an ideal fluid. From the above, Prandtl equations in their finite form will be written as:

$$
\begin{gathered}
\frac{\partial u}{\partial t}+u \frac{\partial u}{\partial x}+v \frac{\partial u}{\partial y}=\frac{\partial U_{e}}{\partial t}+U_{e} \frac{\partial U_{e}}{\partial x}+v\left(\frac{\partial^{2} u}{\partial y^{2}}\right) \\
\frac{\partial u}{\partial x}+\frac{\partial v}{\partial y}=0
\end{gathered}
$$

This is a system of parabolic, nonlinear partial differential equations of the second order which are solved with initial and boundary conditions:

$$
\begin{gathered}
\text { at } t=0, u=u(0, x, y) ; y=0, u=0, v=0 ; \\
y=\delta, u(t, x, y)=U_{e}(t, x) ; x=x_{0}, u=u_{0}(t, y) .
\end{gathered}
$$

The time-averaged differential equation for energy in a given flow field is linear in the temperature if fluid properties are considered to be independent of temperature. Thus, the concept of a Heat Transfer Coefficient arises such that the heat transfer rate from a wall is given by:

$$
q=\alpha\left(T_{w}+T_{r}\right)
$$

where the heat transfer coefficient, $\alpha$, is only a function of the flow field. $T_{w}$ is the wall temperature and $\mathrm{T}_{\mathrm{r}}$, the recovery or adiabatic wall temperature. 
The above is also true of the Boundary Layer energy equation, which is a particular case of the general energy equation. When the wall is at a different temperature to the fluid, there is similarly a small region where the temperature varies. These regions are the velocity and thermal boundary layers. This thin region could be analyzed separately from the bulk fluid flow in that pressure variation normal to the wall may be neglected and the pressure is given by that impressed by the free stream. Velocity normal to the wall is also of order, of the thickness of the boundary layer, the characteristic velocity being that of the free stream and the length being the distance from the leading edge. The boundary layer energy equation for steady incompressible laminar flow in two dimensions may be approximated to be:

$$
u \frac{\partial T}{\partial x}+v \frac{\partial T}{\partial y}=\frac{\lambda}{\rho C_{p}} \frac{\partial^{2} T}{\partial y^{2}}+\mu\left[\frac{\partial u}{\partial y}\right]^{2}
$$

The energy equation may be solved using the Blasius solution to give the heat transfer in terms of the Nusselt Number, $\mathrm{Nu}_{\mathrm{x}}$, when the dissipation term, $\mu(\partial \mathrm{u} / \partial \mathrm{y})^{2}$ is neglected.

$$
N u_{x}=\left[\alpha \frac{x}{\lambda}\right]=\left[C_{f} \frac{R e}{2}\right]
$$

Where, $C_{f}=\frac{2 \tau_{s}}{\rho V^{2}}$ Skin friction, $\tau_{s}=\mu \frac{d V}{d y} \quad$ Shear stress

A heat transfer coefficient $\mathrm{h}$ is generally defined as:

$$
Q=h A\left(T_{s}-T_{\infty}\right)
$$

Where $Q$ is the total heat transfer, $A$ is the heated surface area, $T_{s}$ is the surface temperature and $\mathrm{T}_{\infty}$ is the approach fluid temperature. For the convection heat transfer to have a physical meaning, there must be a temperature difference between the heated surface and the moving fluid. This phenomenon is referred to as the thermal boundary layer that cause heat transfer from the surface. In addition to the thermal boundary layer, there is also a velocity boundary layer due to the friction between the surface and the fluid induced as the result of the fluid viscosity. The combination of the thermal and viscous boundary layers governs the heat transfer from the surface. 


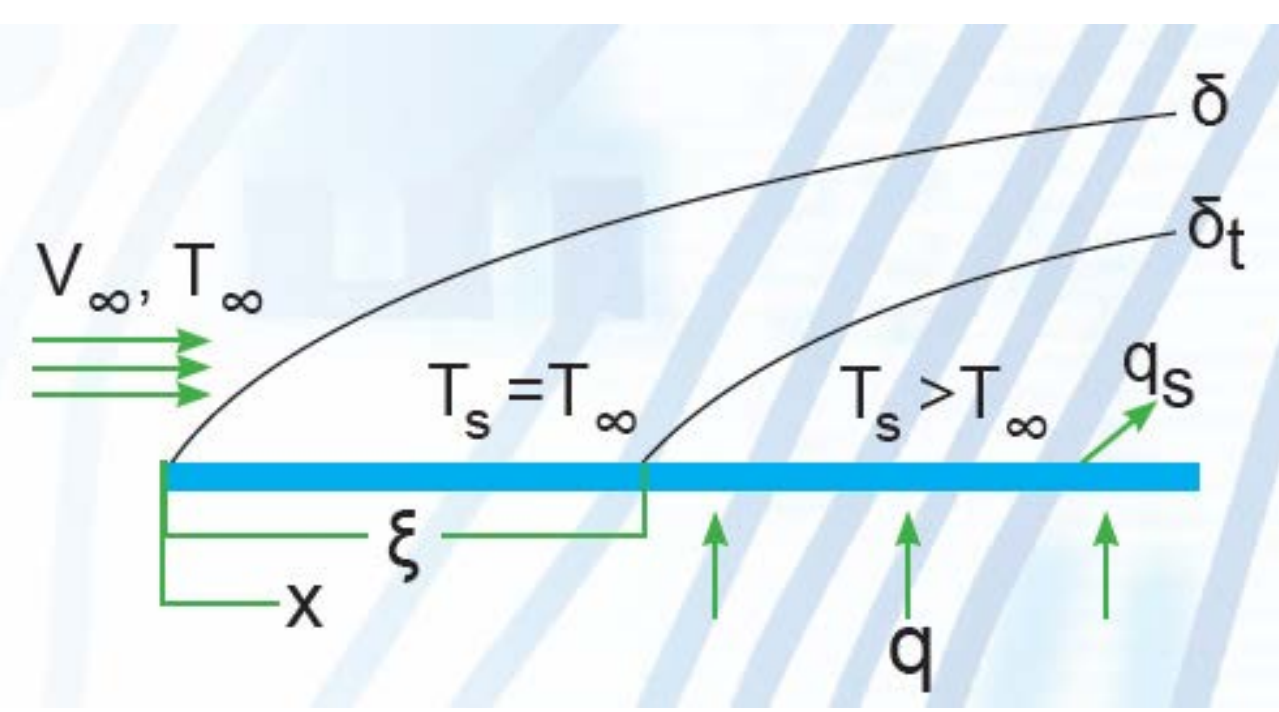

Figure 3 Velocity boundary layer growth $(\delta)$ that starts from the leading edge of the plate. The thermal boundary layer $\left(\delta_{t}\right)$ starts after a distance $(\xi)$ from where the temperature of the plate changes from ambient temperature to a different temperature $\left(T_{s}\right)$, causing convection heat transfer.

\subsubsection{Heat Transfer Coefficient Relation with Reynolds number}

Heat transfer coefficient purely depends on the fluid and surface reference temperatures. In simple geometries, $\mathrm{h}$ can be analytically derived based on the conservation equations. For the laminar flow over a flat plate, analytical derivation for the local heat transfer coefficient yields

$$
h_{x}=\frac{0.332}{x} k_{f} \operatorname{Re}_{x}^{1 / 2} \operatorname{Pr}^{1 / 3}
$$

The heat transfer coefficient also depends on the flow regime. Figure 3 shows the flow over a flat surface. The laminar boundary layer starts the transition at a Reynolds number around $5 \times 10^{5}$ with a sudden jump in the heat transfer coefficient, and then gradually coming down in the turbulent region, but still above the laminar region. For a smooth circular tube, the transition from laminar to turbulent starts at a Reynolds number around 2300. 


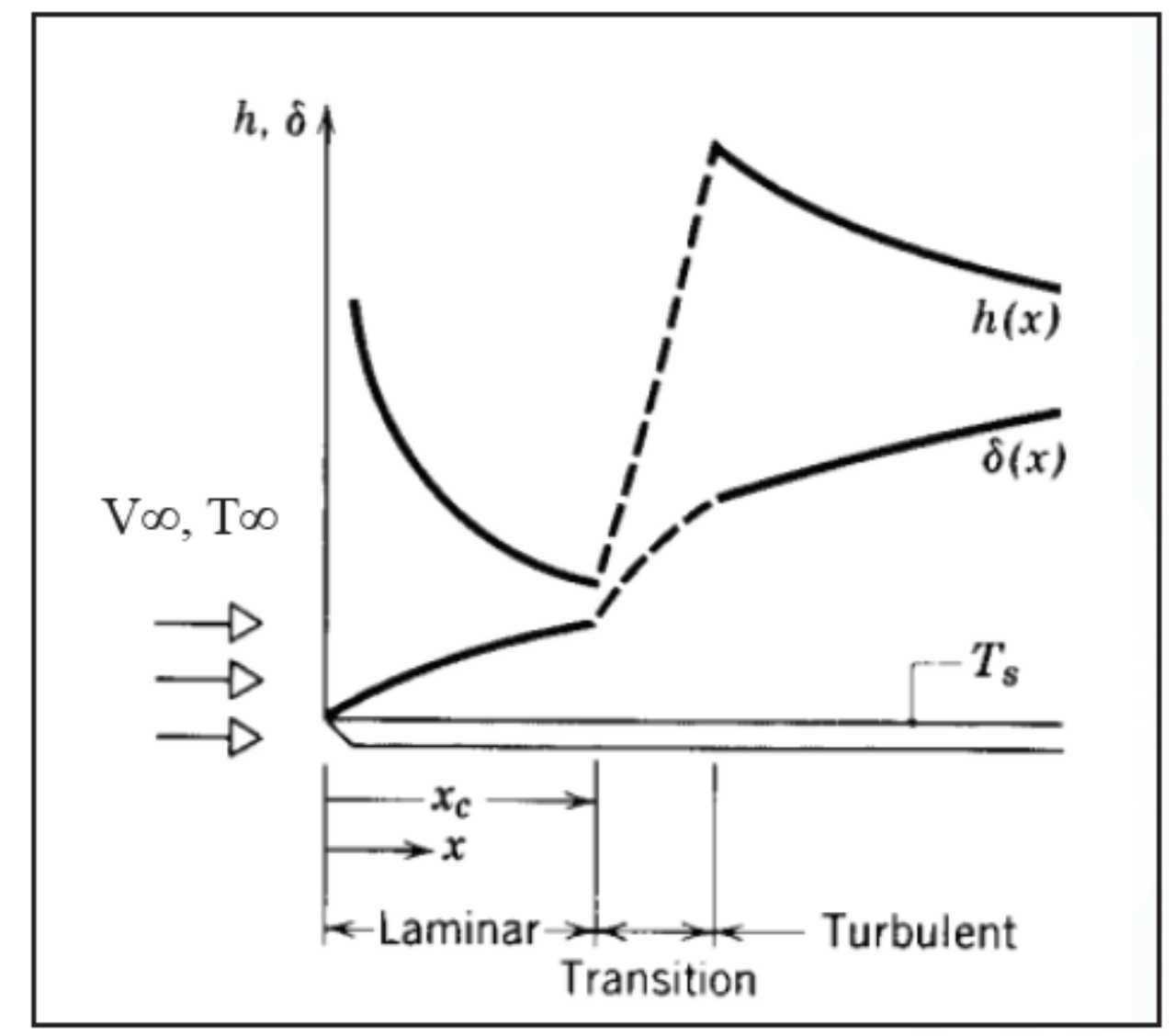

Figure 4 Heat Transfer Coefficient on a Flat plate for different flow regime

\subsection{Introduction to Nanofluids}

A nanofluid is a fluid containing nanometer (10-9 m) sized particles, called nanoparticles (particles between 1 and 100 nanometers in size). These fluids are engineered colloidal suspensions of nanoparticles in a base fluid. A colloid has a dispersed phase (the suspended particles) and a continuous phase (the medium of suspension). The nanoparticles used in nanofluids are typically made of metals, oxides, carbides, or carbon nanotubes. Common base fluids include water, ethylene glycol and oil. In analysis such as computational fluid dynamics (CFD), nanofluids can be assumed to be single phase fluids. However, almost all new academic paper uses two-phase assumption. Classical theory of single phase fluids can be applied, where physical properties of nanofluid is taken as a function of properties of both constituents and their concentrations [14]. An alternative approach simulates nanofluids using a two-component model [15]. 


\subsubsection{Synthesis of Nanofluids}

Fabrication of nanoparticles can be categorized into major two broad categories: Chemical process and Physical process. Conventionally physical methods include inert-gas condensation, mechanical grinding etc. Some of the chemical methods are chemical precipitation, micro emulsion, thermal spray, spray pyrolysis etc. Dispersion of Nano particles in liquid i.e. nanofluid preparation are produced mainly by two methods: Two-step technique and Single-step technique.

The two-step method first makes nanoparticles using one of the above described nanoparticle processing techniques and then disperses them into base fluids.

The single-step method simultaneously makes and disperses nanoparticles directly into the base fluids.

In either case, a well mixed and uniformly dispersed nanofluid is needed for successful production or reproduction of enhanced properties and interpretation of experimental data. Nanofluids are produced by several techniques they are, 1 . Direct Evaporation (Single-step), 2. Gas condensation/dispersion (Two-step), 3. Chemical vapour condensation (Single-step), 4. Chemical precipitation (Single-step), etc.

\subsubsection{Thermal Conductivity of Oxide Nanofluids}

It is a well-known fact that the thermal conductivity of the suspensions is higher than that of the base fluid. This is primarily because the solids have higher thermal conductivity then liquids, except for liquid metals. When nanofluids were invented by Choi and hiss team members at the Argonne National Laboratory, they first tried to use oxide particles of nanometer size to suspend in the common coolants [16]. In another major publication, in which Choi and his team described the transient hotwire method to measure the thermal conductivity of $\mathrm{Al}_{2} \mathrm{O}_{3}$ and $\mathrm{CuO}$ nanoparticles suspended in water and ethylene glycol [17]. First, they found that the enhancement of thermal conductivity is linear, see figure 5. 


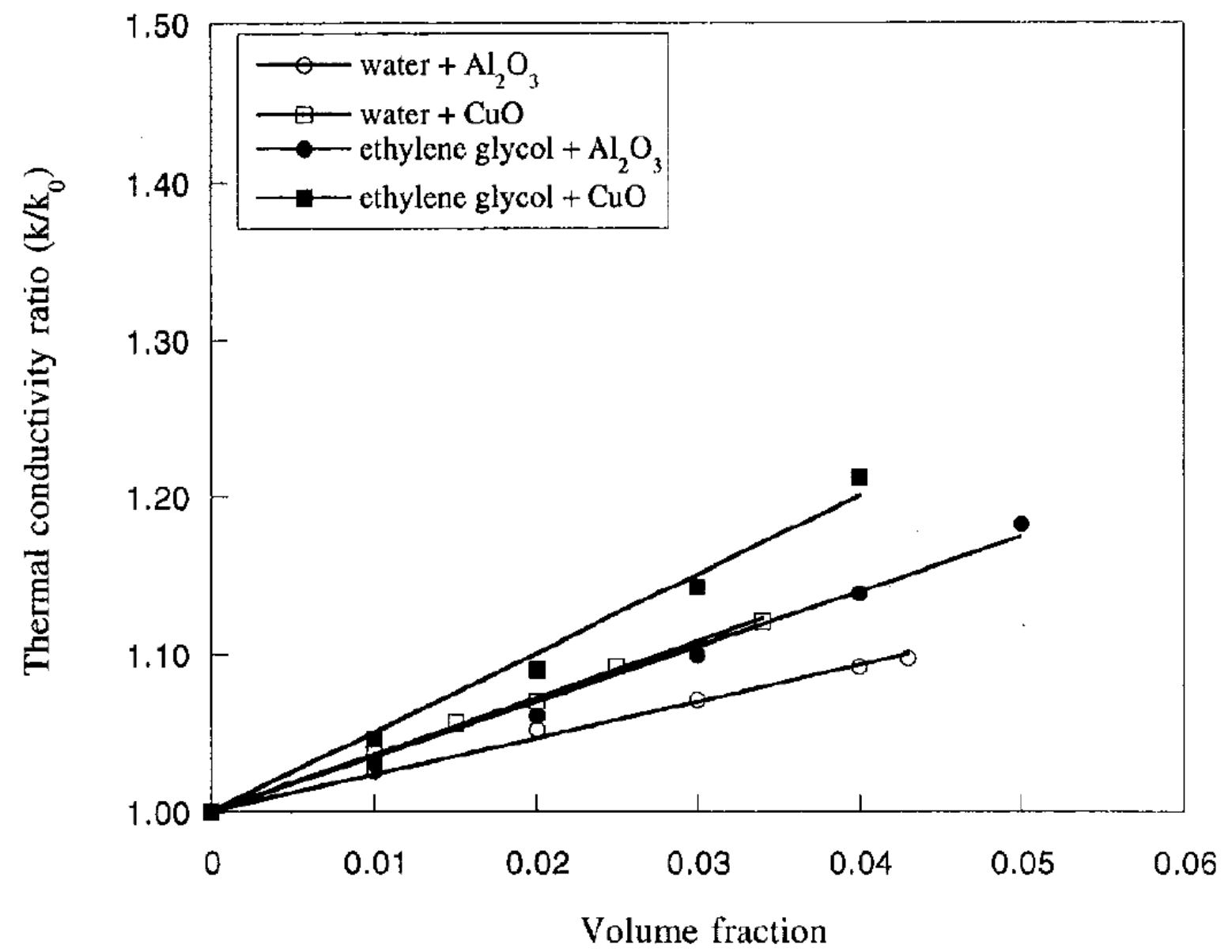

Figure 5 Enhanced thermal conductivity of Oxides nanofluids system [17]

They chose the modified Maxwell theory of Hamilton \& Crosser [18] as the basis for comparing the experimental results. This theory gives the enhancement of thermal conductivity of suspension in the form

$$
\boldsymbol{k}_{\text {eff }}=\boldsymbol{k}_{0} \frac{\boldsymbol{k}_{p}+(n-1) k_{0}-(n-1) \varepsilon\left(k_{0}-k_{p}\right)}{k_{p}+(n-1) k_{0}+\varepsilon\left(k_{0}-k_{p}\right)}
$$

Where $k_{\text {eff }}$ is the effective conductivity of the nanofluid, $k_{p}$ the particle conductivity, $\mathrm{k}_{0}$ the base fluid conductivity, the particle volume fraction, and $\mathrm{n}$ the particle shape factor $\mathrm{n}=\frac{3}{\psi}, \psi$ is the sphericity of the particles. Comparison showed good agreement, for both water and ethylene glycol. 


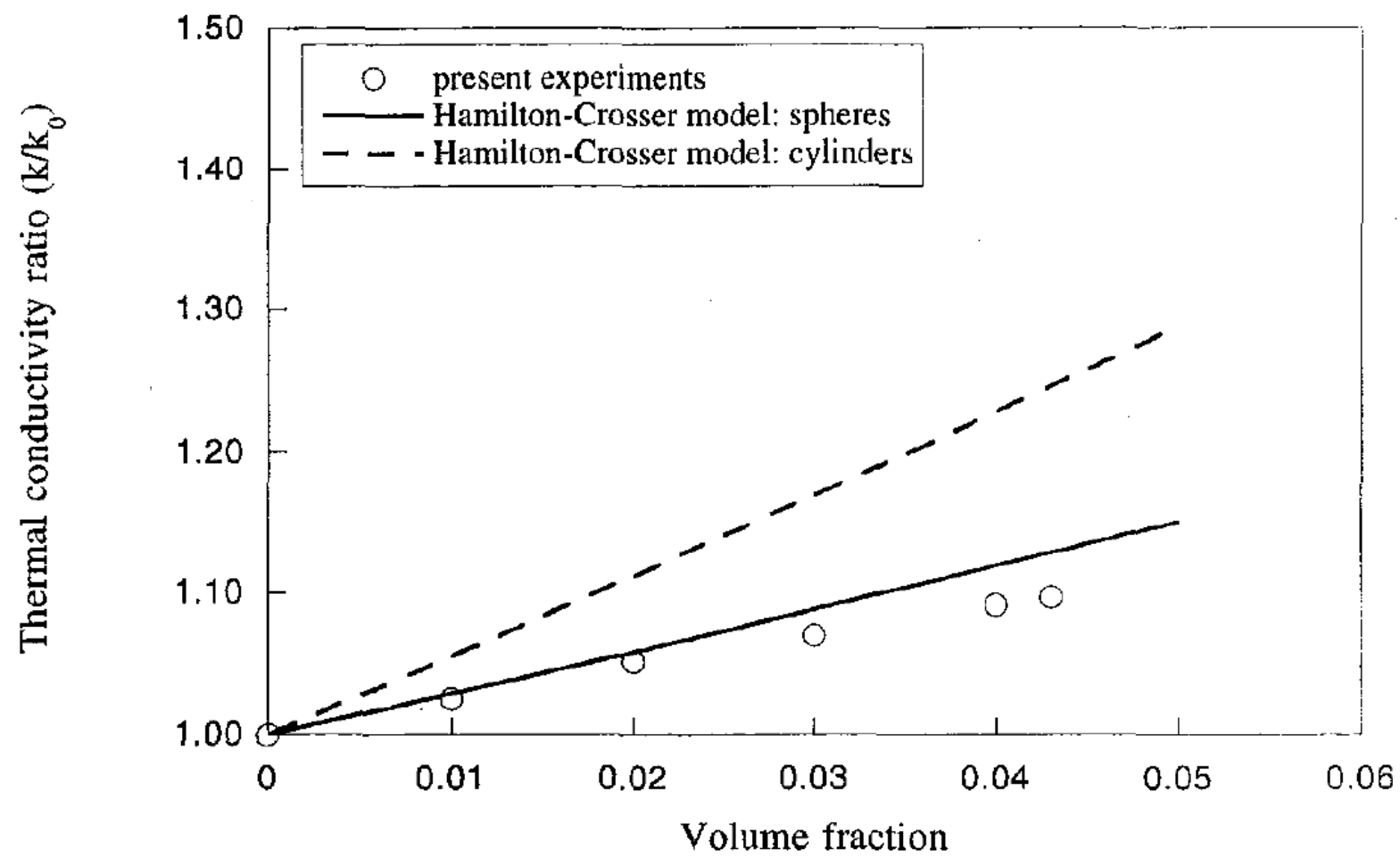

Figure 6 Comparison of $\mathrm{Al}_{2} \mathrm{O}_{3}$-Water nanofluid conductivity using Hamilton-Crosser theory [18]

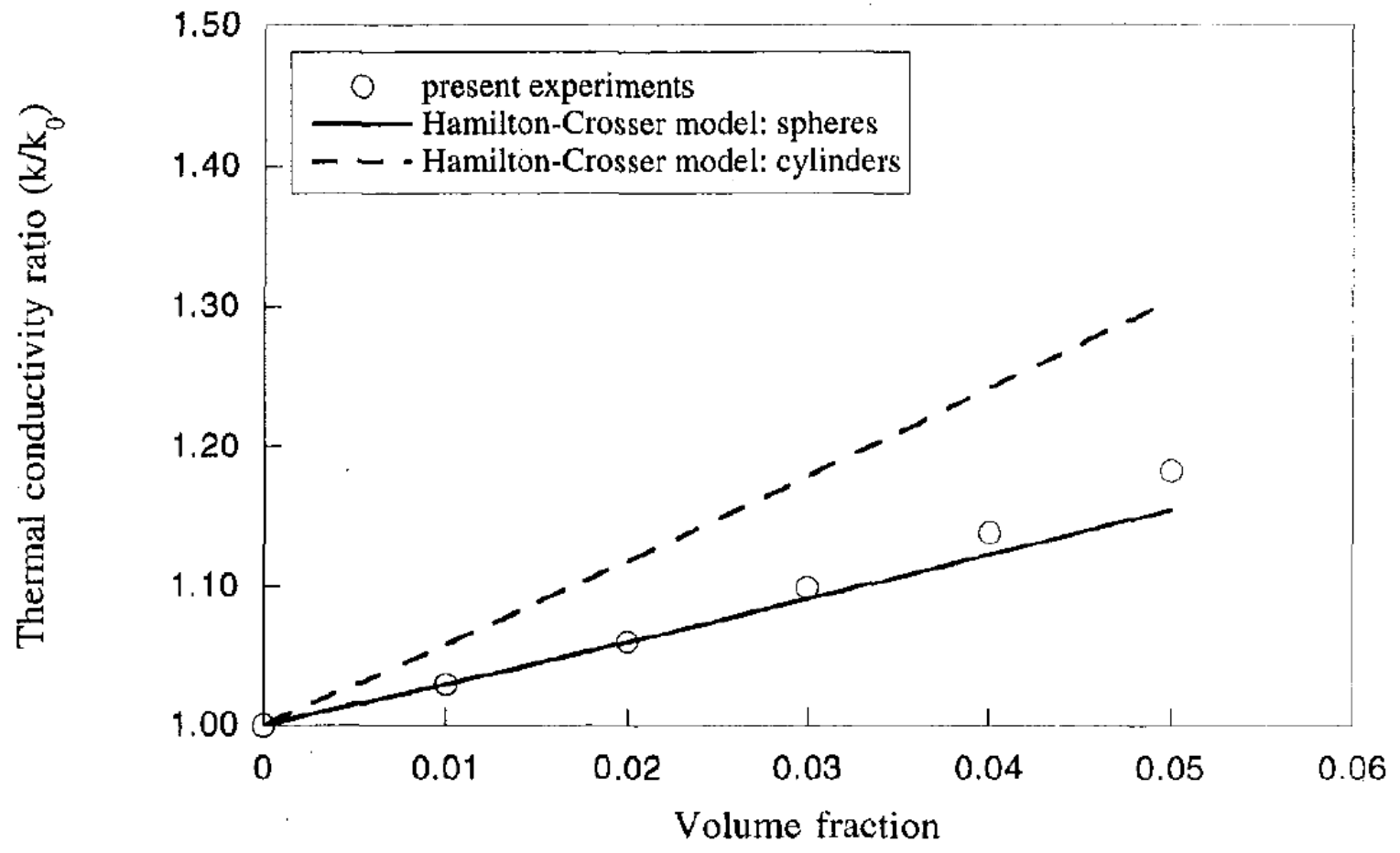

Figure 7 Comparison of $\mathrm{Al}_{2} \mathrm{O}_{3}$-Ehylene glycol nanofluid conductivity using Hamilton-Crosser theory [18] 
When experiment was conducted using CuO-particle based nanofluid, results were astonishing, thermal conductivity were much higher then Hamilton-Crosser theory prediction, in both the cases water and ethylene glycol, see figure $8 \& 9$. The average particle size used, for the experiment, for $\mathrm{Al}_{2} \mathrm{O}_{3}$ was $38 \mathrm{~nm}$ and $24 \mathrm{~nm}$ for $\mathrm{CuO}$, which introduces the possibility of nanoparticle size effect.

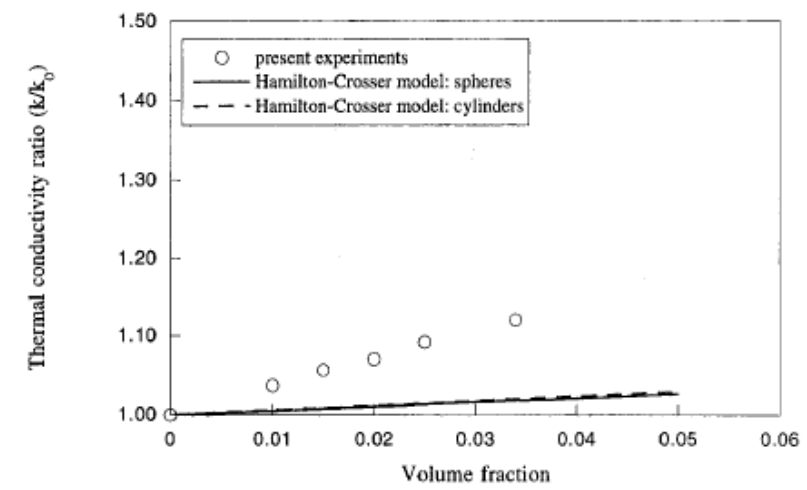

Figure 8 Comparison of CuO-Water nanofluid conductivity using Hamilton-Crosser theory [18]

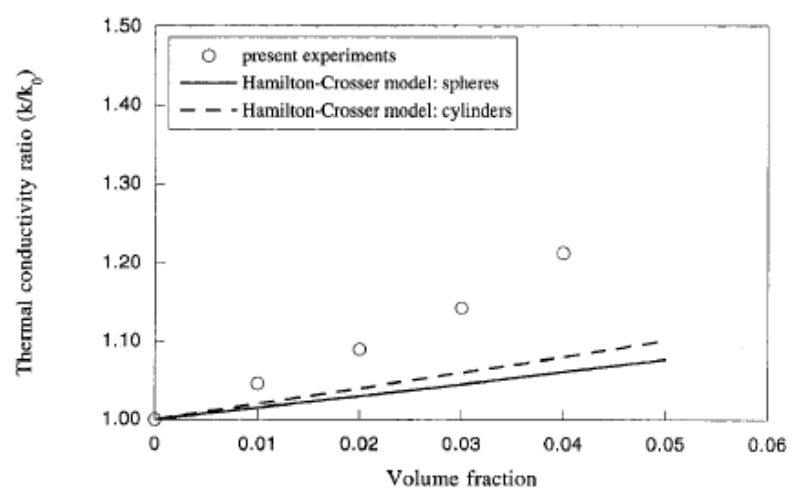

Figure 9 Comparison of CuO-Ethylene glycol nanofluid conductivity using Hamilton-Crosser theory [18]

$\mathrm{Al}_{2} \mathrm{O}_{3}$ nanofluid results were also compared with those of Masuda \& Ebata experiment [19], in which $\mathrm{Al}_{2} \mathrm{O}_{3}$ nanofluid with $13 \mathrm{~nm}$ particle size, showed much higher thermal conductivity than when using Hamilton-Crosser model, see figure 10. These results seem to indicate that the nanoparticle size effect maybe the reason for the abnormal increase in the thermal conductivity of nanofluids. 


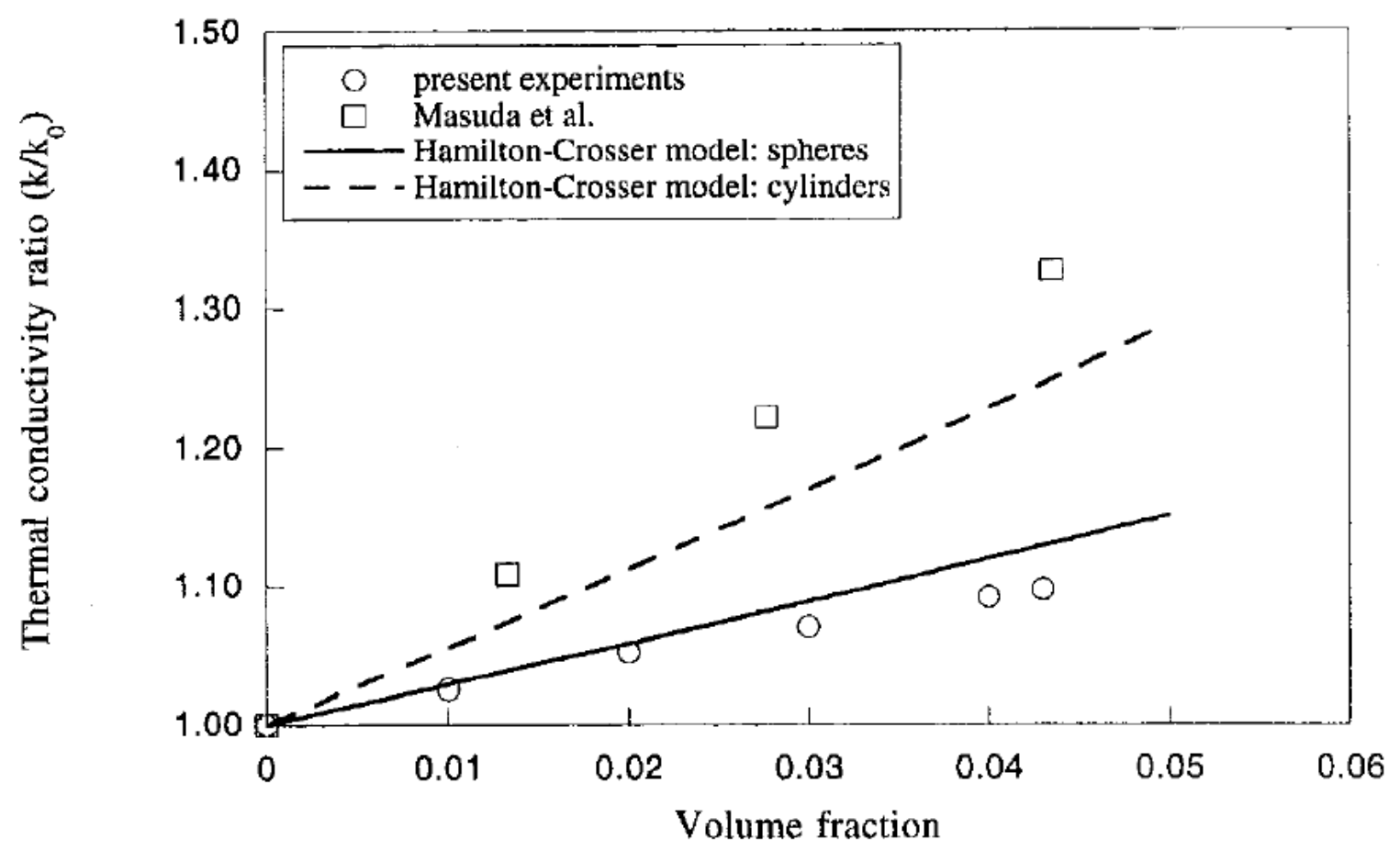

Figure 10 comparison of $\mathrm{Al}_{2} \mathrm{O}_{3}$-Water Nanofluid conductivity from Lee-Choi \& Masuda experiments [17][19]

Thus, with a decrease in particle size, the surface area of nanoparticle increases, giving more heat transfer area between the phases, which may be helpful in increasing the thermal transport.

Jang and Choi [20] have developed, for the first time, a dynamic model that takes into account convection induced by a single Brownian nanoparticle. They derived a general expression for thermal conductivity of nanofluids involving four modes of energy transport. The first mode is collision between the base fluid molecules (i.e. thermal conductivity of the base fluid). The second mode is the thermal conductivity of the nanoparticles in the nanofluid. The third mode is collision between nanoparticle due to Brownian motion, which can be neglected because Brownian diffusion of nanoparticle is a very slow process compared to thermal diffusion. The last mode is thermal interaction of dynamic nanoparticles with base fluid molecules. Forth mode is a key to temperature and size dependent conductivity. The effective thermal conductivity of nanofluid is given by

$$
\boldsymbol{k}_{\text {eff }}=\boldsymbol{k}_{B f}(1-f)+\boldsymbol{k}_{\text {nano }} f+3 C_{1} \frac{d_{B f}}{d_{\text {nano }}} \boldsymbol{k}_{B f} \boldsymbol{R}_{d_{\text {nano }}}^{2} \operatorname{Prf}
$$

Where keff, $f, k_{\text {nano, }}, \mathrm{C} 1, \mathrm{~d}_{\mathrm{Bf}}$, Pr are Base Fluid conductivity, volume fraction of nanoparticles, thermal conductivity of nanoparticles, empirical constant, diameter of 
base fluid particle, diameter of the nanoparticle, and Prandtl number, respectively. Reynolds number nanofluid $R e_{d_{\text {nano }}}=\frac{\overline{\bar{C}_{R . M .}} d_{\text {nano }}}{\mathcal{V}}$,

Random motion velocity $\overline{C_{R . M .}}=\frac{D_{0}}{l_{B f}}, l_{B f}=$ Mean free path Base-Fluid molecule, Nanoparticle diffusion coefficient $D_{0}=\frac{k_{b} T}{3 \pi \mu d_{\text {nano }}}$, Boltzmann constant $k_{b}=1.3870$ $\mathrm{x} 10^{-23} \mathrm{~J} / \mathrm{K}, \mu=$ Viscosity of Base fluid, $\mathrm{T}=$ temperature of Base fluid.

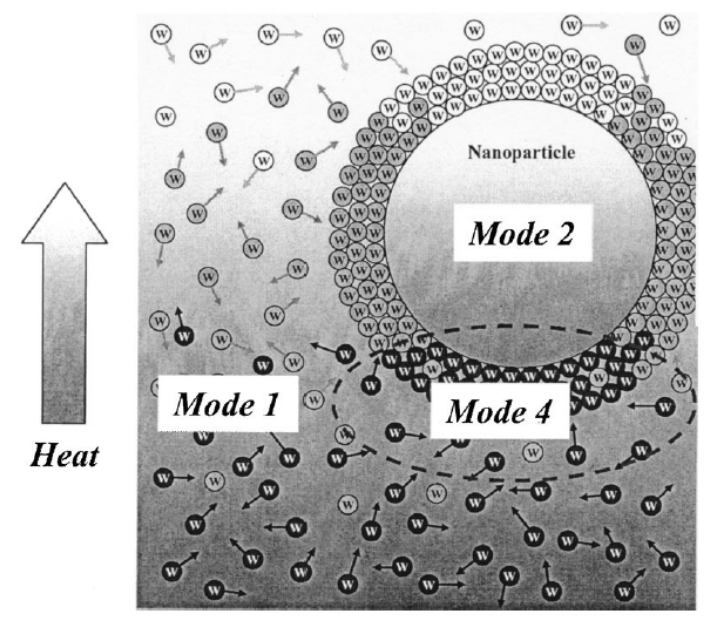

Figure 11 Modes of energy transport in Nanofluids [20]

\subsubsection{Convection in Nanofluids}

Although number of studies available is very limited for this area, compared to convection, there are some very significant findings worth mentioning in this section. Convective heat transfer is closely related to the viscosity of suspension. The model of Batchelor [21] is applicable to dilute fluids dispersed with a solid sphere, given by:

$$
\mu_{r}=1+2.5 \varnothing+6.2 \emptyset^{2}
$$

Where $\mu_{\mathrm{r}}$ is relative viscosity of the suspension and $\varnothing$ is particle volume fraction. Often simplified form of this equation known as Einstein equation is used to estimate the viscosity of dilute suspensions.

$$
\mu_{n f}=\mu_{b f}(1+2.5 \emptyset)
$$


where $\mu_{\mathrm{bf}}=$ base fluid viscosity.

Pak and Cho [22] proposed a Dimensionless Nusselt number equation, which was modified from Dittus-Boelter correlation [23]

$$
N u=0.021 R e^{0.8} \operatorname{Pr}^{0.5}
$$

Reynolds number of a nanofluid mixture, from planer jet is defined as:

$$
\boldsymbol{R} \boldsymbol{e}_{n f}=\frac{\rho_{n f} U D}{\mu_{n f}}
$$

where $\mathrm{U}$ and $\mathrm{D}$ are the fluid velocity and the width of planar jet at the impingement respectively. Density of a mixture, can be calculated according to the following equation [24]

$$
\rho_{n f}=(1-\varnothing) \rho_{b f}+\emptyset \rho_{n p}
$$

where $u$ indicates the volume fraction of nanoparticles, $\rho_{\mathrm{np}}$ and $\rho_{\mathrm{bf}}$ are respectively the densities of nanoparticles and base fluid. Heat capacity of the nanofluid is calculated using the following equation

$$
\left(\rho C_{P}\right)_{n f}=\varnothing\left(\rho C_{P}\right)_{n p}+(1-\varnothing)\left(\rho C_{P}\right)_{b f}
$$

where $\mathrm{u}$ indicates the volume fraction of nanoparticles, $\mathrm{C}_{\mathrm{p}, \mathrm{nf}}$ is the heat capacity of nanofluid, also $C_{p, n p}$ and $C_{p, b f}$ are heat capacities of nanoparticles and base fluid respectively. The local Nusselt number, $\mathrm{Nu}$, is defined as,

$$
N u=\frac{h D}{k_{n f}}
$$

where $k_{n f}$ is the thermal conductivity of the nanofluid, which can be calculated theoretically as the following

$$
k_{n f}=\frac{k_{n p}+2 k_{b f}-2 \varnothing\left(k_{b f}-k_{n p}\right)}{\frac{k_{n p}}{k_{b f}}+2+\varnothing\left(\frac{k_{b f}-k_{n p}}{k_{b f}}\right)}
$$

Where $\mathrm{k}_{\mathrm{np}}$ is the thermal conductivity of the nanoparticles, $\mathrm{k}_{\mathrm{bf}}$ is the thermal conductivity of the base fluid. 


\section{CHAPTER 3}

\subsection{Numerical Model Background}

Two-equation models allow the determination of both, a turbulent length and time scale by solving two separate transport equations. The k- $\varepsilon$ model in ANSYS Fluent falls within this class of models and has become the workhorse of practical engineering flow calculations.

Knudsen number is defined as the ratio of the molecule mean free path of base fluid molecules to the nanoparticles diameter

$$
k_{n}=\frac{\lambda}{d_{p}}
$$

where $d_{p}$ is the particle diameter and $\lambda$ is the molecule mean free path of base fluid molecules and is given by:

$$
\lambda=\frac{R * T}{\pi * d_{m} * N_{A} * P \sqrt{2}}
$$

where $R$ is the universal gas constant, $T$ is the temperature, $d_{m}$ is the molecular diameters of base fluid, $\mathrm{N}_{\mathrm{A}}$ is the Avogadro's constant, and $\mathrm{P}$ is the atm. pressure.

For this experiment, Commercial spherical-shape Al2O3 powder with 99.5\% purity and $15 \mathrm{~nm}$ average diameter was used. Molecule mean free path of base fluid molecules (i.e. water) is,

$$
\lambda=\frac{8.314 * 288.15}{\pi *(2.7 e-10) *(6.022 e 23) * 101325 * \sqrt{2}}=3.27297 e-17 \mathrm{~m}
$$

Knudsen number can be calculated as,

$$
k_{n}=\frac{\lambda 3.27297 e-17}{1.5 e-8}=2.1819 e-9
$$

Therefore, for the nanoparticles in range of interest $(1-100 \mathrm{~nm})$, the Knudsen number is relatively small $\left(\mathrm{k}_{\mathrm{n}}<0.1\right)$; thus, the assumption of continuum is reasonable. [25] 
Robustness, economy, and reasonable accuracy, of this model, for a wide range of flows explain its popularity in industrial flow and heat transfer simulations.

Most commercial software uses the Reynolds Averaged Navier-Stokes equations (RANS). RANS system is available with $\mathrm{k}-\varepsilon$ in Ansys FLUENT. It takes less computational effort than the time accurate equations and is robust for a wide range of fluid flows. It is derived from the standard equations by averaging after decomposing the flow variables into mean and fluctuating components like $t=\bar{t}+$ $t^{\prime}$, where $\bar{t}$ is the mean (time averaged) and $t^{\prime}$ is the fluctuating component of variables like velocity, pressure or other scalar quantity. This formulation leads to the continuity and momentum equations as follows

$$
\begin{gathered}
\frac{\partial \rho}{\partial t}+\frac{\partial\left(\rho \overline{u_{\imath}}\right)}{\partial x_{i}}=0 \\
\frac{\partial\left(\rho \overline{u_{\imath}}\right)}{\partial t}+\frac{\partial\left(\rho \overline{u_{\iota}} \overline{u_{J}}\right)}{\partial x_{j}}=\frac{-\partial \bar{P}}{\partial x_{i}}+\frac{\partial\left[\mu\left(\frac{\partial \overline{u_{l}}}{\partial x_{j}}+\frac{\partial \overline{u_{J}}}{\partial x_{i}}-\frac{2}{3} \delta_{i j} \frac{\partial \overline{u_{l}}}{\partial x_{l}}\right)\right]}{\partial x_{j}}+\frac{\partial\left(-\rho \overline{u_{l}{ }_{l} u_{\jmath}}\right)}{\partial x_{j}}
\end{gathered}
$$

This study requires to solve the energy equation with the $\mathrm{k}-\varepsilon$ model. The energy equation is [25]

$$
\frac{\partial(\rho E)}{\partial t}+\frac{\partial\left[u_{i}(\rho E+P)\right]}{\partial x_{i}}=\frac{\partial\left(k_{e f f} \frac{\partial T}{\partial x_{j}}+u_{i} \tau_{i j} e_{e f f}\right)}{\partial x_{i}}+S_{h}
$$

Where, $\mathrm{E}$ is the Total Energy, $\mathrm{T}$ is temperature, $\mathrm{k}$ is thermal conductivity, $\mathrm{k}_{\mathrm{eff}}=\mathrm{k}+$ $\left(\mathrm{C}_{\mathrm{p}} \mu_{\mathrm{t}} / \mathrm{Pr}_{\mathrm{t}}\right)$ the effective thermal conductivity, $\mathrm{Pr}_{\mathrm{t}}$ - Turbulent Prandtl number, $\tau_{\text {eff }}$ the deviatoric stress tensor and $S_{h}$ is source term.

The impingement of water jet is a free surface flow which was modeled by the Volume of Fluid method (VOF). It calculates the volume fraction of liquid in all the control volumes (or, cells) and depending on the value of the volume fraction it finds the cells that contain the interface. If the volume fraction $\alpha_{\mathrm{q}}$ for phase $\mathrm{q}$ is 0 then the cell is empty, if 1 then the cell is full but if $0<$ $\alpha_{q}<1$ then the cell contains the interface. Then a method called Geometric Reconstruction can be used to approximate the interface in those cells containing the interface by a piecewise linear interpolation approach. Another option could be to solve the VOF equation with the Level-Set method. 


\subsection{1 k- $\varepsilon$ model for Mixed Regime Jet flow}

The flow transition from laminar (at inlet) to turbulent (at plate) depends on the surface geometry, surface roughness, upstream velocity, surface temperature, and the type of fluid, and various other factors like surface temperature boundary layer thickness, density etc. [27]. k- $\varepsilon$ model will solve two additional equations for turbulent kinetic energy, $\mathrm{k}$ and its dissipation, $\varepsilon$, respectively, which are both zero for low velocities and less energy. Parameters relate to low momentum and diffused through molecular collisions. No generation of new length scales, because flow has small kinetic energy. Flow streamlines in figure 12, at corner and at tip (stagnation point) shows the above-mentioned trait of fluid, justifies current numerical model. Boundary layer thickness,

$$
\delta=\frac{0.38 x}{R e_{x}^{\frac{1}{5}}}
$$

Where $\mathrm{x}$ is the distance from the leading edge, i.e. stagnation point, $\mathrm{Re}_{\mathrm{x}}$ Reynolds number at location $\mathrm{x}, \delta$ is boundary layer thickness.

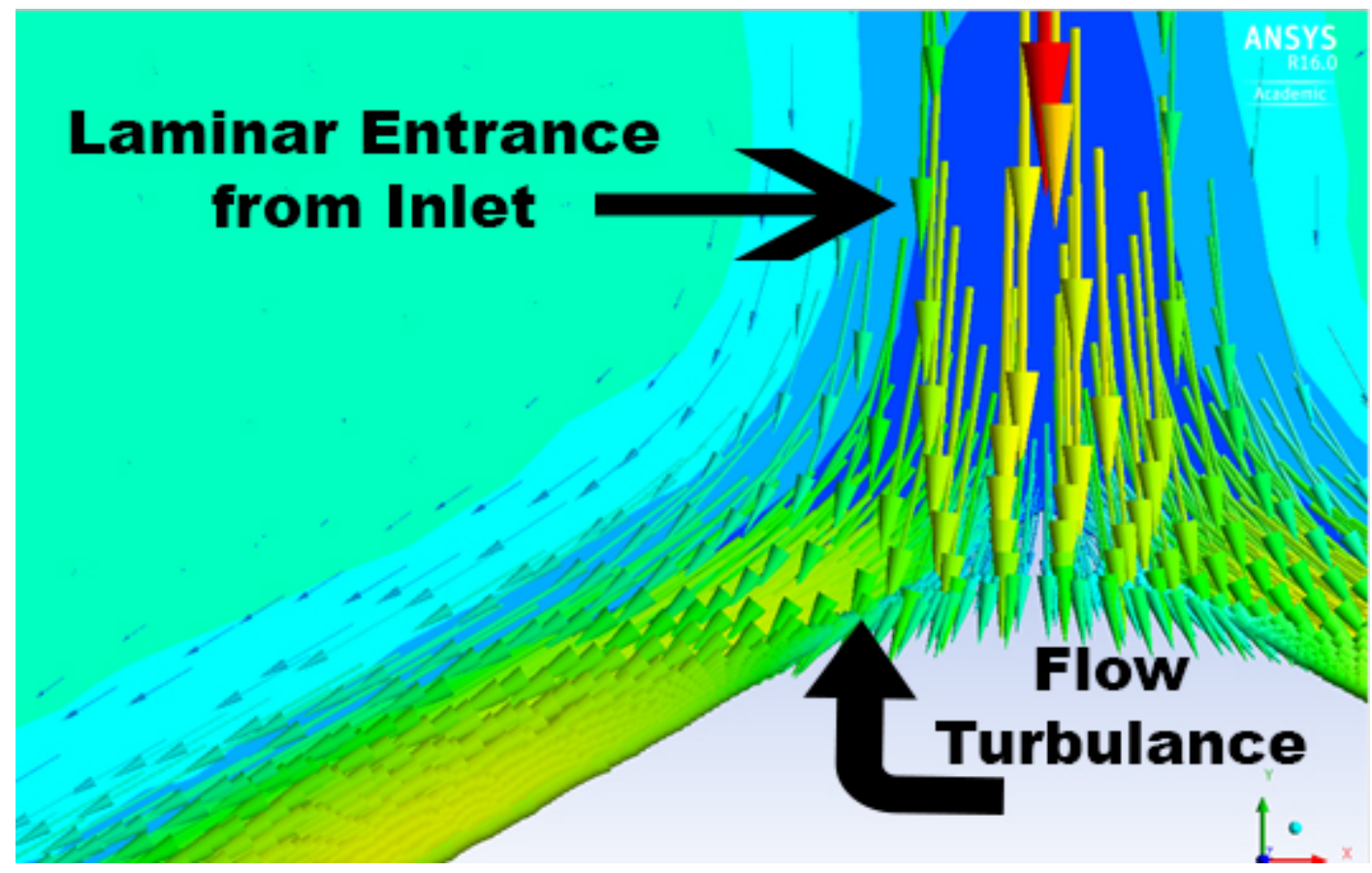

Figure 12 Flow streamlines 


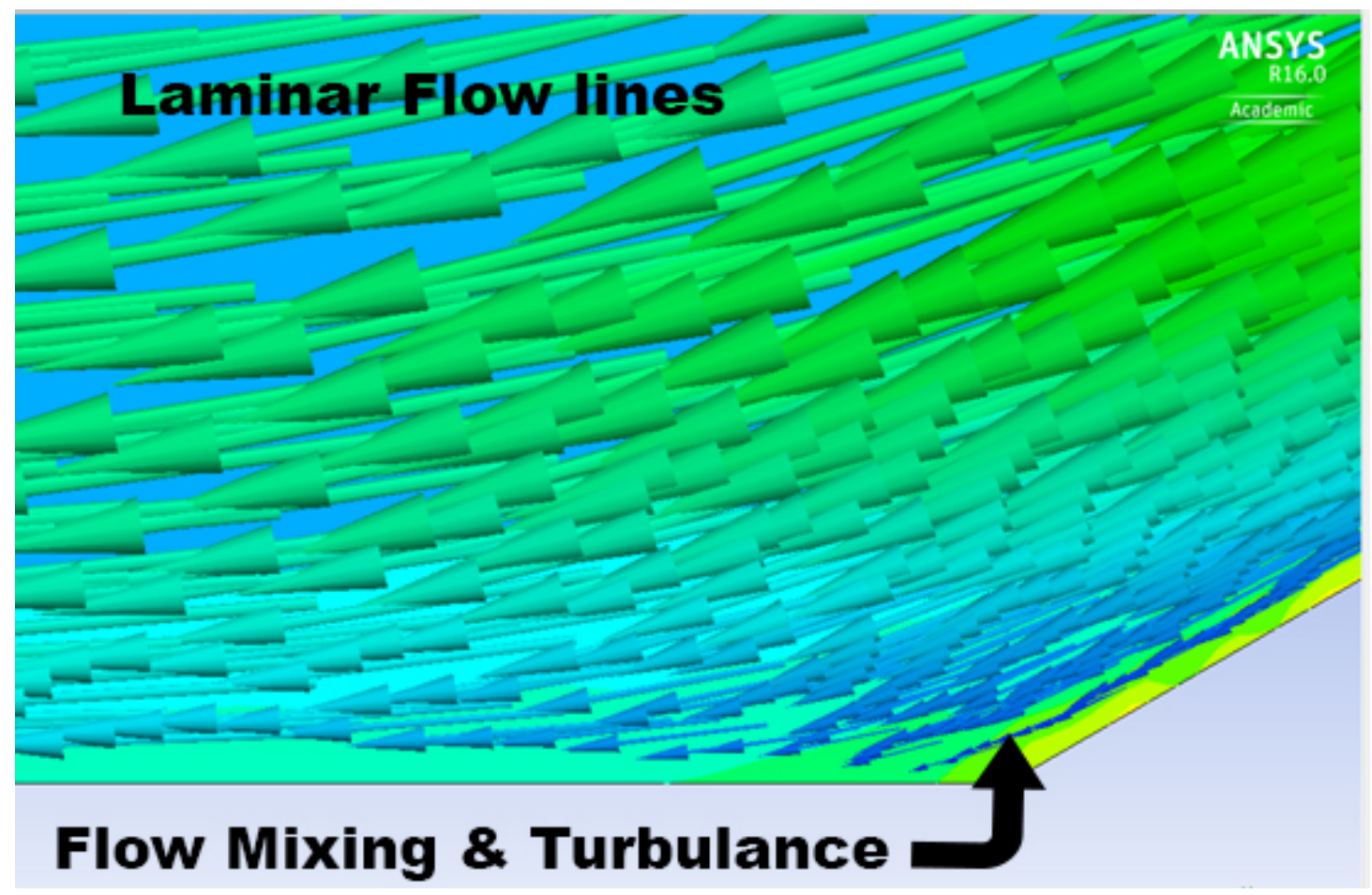

Figure 13 Flow streamlines

\subsection{Computational domain and Mesh}

The computational domain geometry and mesh were built using Ansys Workbench 16.0. The domain was chosen as small as possible to minimize the number of mesh elements. Figure 12 shows the graphical geometry and the boundary conditions and Figure 13 shows the geometry for the 2D planer model, designed in Ansys DesignModeler.

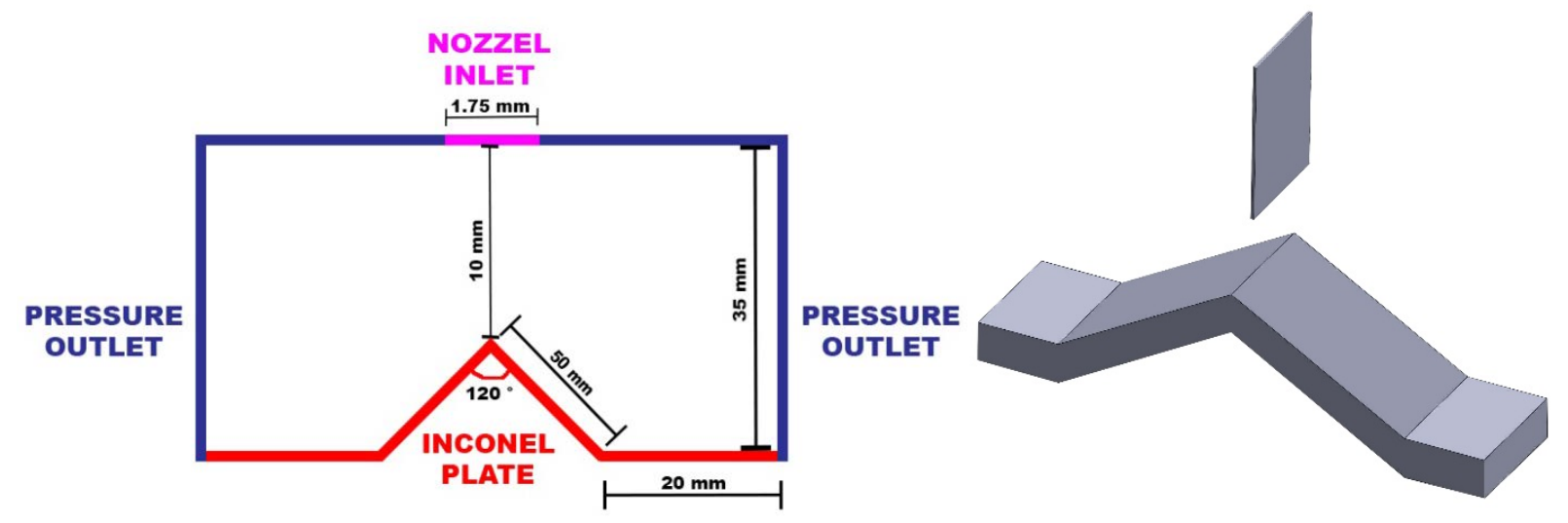

Figure 14 Graphical representation of Geometry: 2D (Left), 3D(Right) 


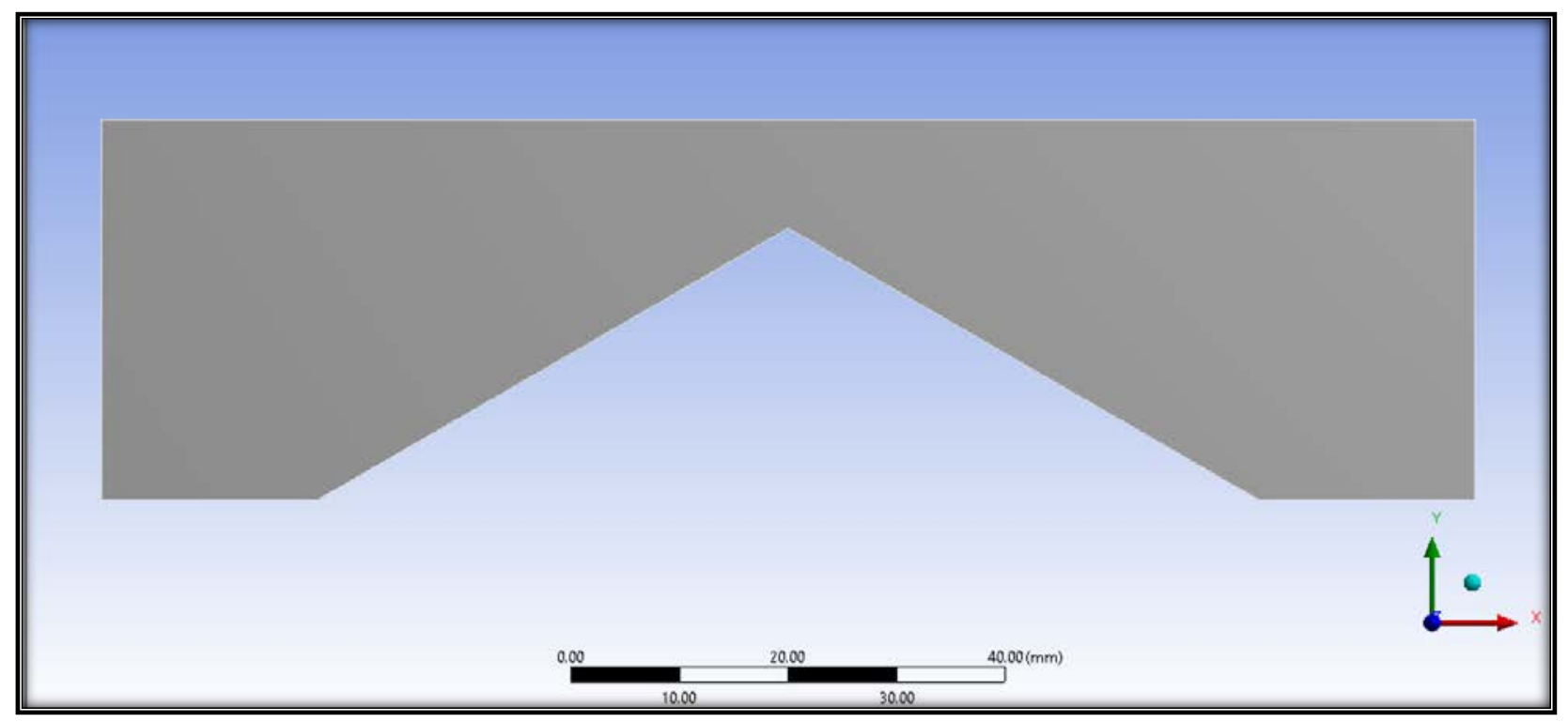

Figure 15 ANSYS Fluent DesignModeler

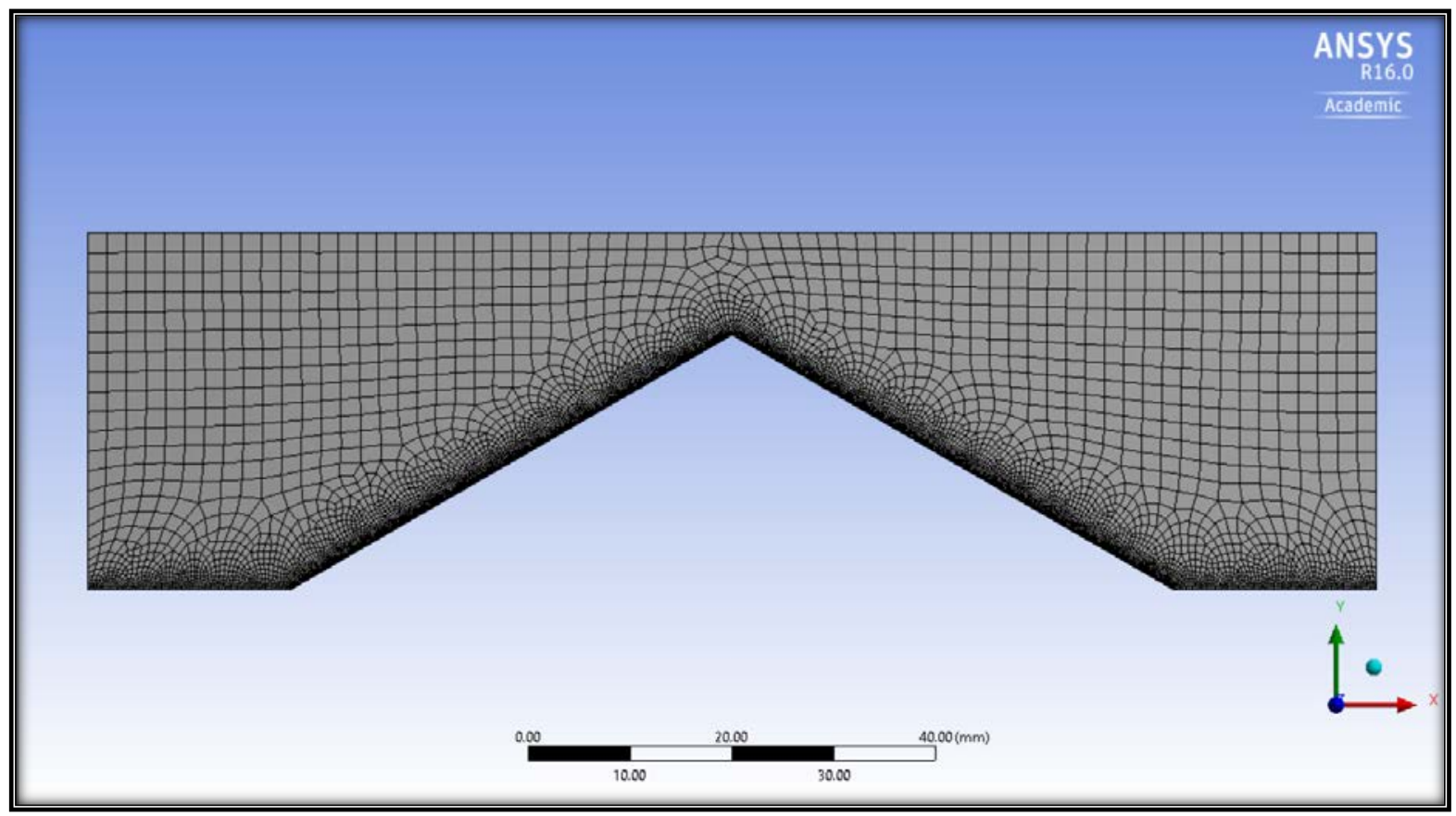

Figure 16 ANSYS Fluent Meshing

A very fine mesh was generated around the boundary layer at the plate surface. The mesh quality is presented in table. The mesh for the jet model is presented in Figure 14. The mesh statistics \& sizing is presented in table. The quality of mesh is decided 
depending on some properties of the cells in FLUENT. In table, different column shows different properties where the aspect ratio is the ratio of the largest and smallest edge of a cell. The mesh quality is fine and high smoothing, to get very precise results.

\begin{tabular}{r|c}
\multicolumn{1}{c}{ Statistics } \\
\hline Nodes & 11185 \\
\hline Elements & 10464 \\
\hline Mesh Metric & Aspect Ratio \\
\hline Max & 1.0003 \\
\hline Average & 2.1953 \\
\hline Standard Deviation & 1.2642 \\
\hline Sizing & 0.18209 \\
\hline Advanced Size Function & On: Curvature \\
\hline Relevance Center & Fine \\
\hline Initial Size Seed & Active Assembly \\
\hline Smoothing & High \\
\hline Span Angle Center & Fine \\
\hline Curvature Normal Angle & Default (18.0 $\left.{ }^{\circ}\right)$ \\
\hline Min Size & Default (1.9175e-002 mm) \\
\hline Max Face Size & Default (1.91750 mm) \\
\hline Max Size & Default (3.83510 mm) \\
\hline Growth Rate & Default $(1.20)$ \\
\hline Minimum Edge Length & $0.7650 \mathrm{~mm}$
\end{tabular}

Table 1 Mesh statistics \& sizing

\begin{tabular}{|c|c|c|c|c|}
\hline Mesh & Elements & $\begin{array}{l}\text { Average heat transfer } \\
\text { coefficient } \\
\text { (Numerical study) } \\
\overline{h_{\text {num }}}\end{array}$ & $\begin{array}{l}\text { Average heat transfer } \\
\text { coefficient } \\
\text { (Experimental study) } \\
\overline{h_{\text {exp }}}\end{array}$ & $\begin{array}{c}\text { Variation \% } \\
\frac{\overline{h_{\text {num }}}-\overline{h_{\text {exp }}}}{\overline{h_{\text {exp }}}} \times 100\end{array}$ \\
\hline Coarse & 9969 & 9512 & 8617 & $10.38 \%$ \\
\hline Medium & 10083 & 9467 & 8617 & $9.86 \%$ \\
\hline Fine & 10464 & 9310 & 8617 & $8.04 \%$ \\
\hline
\end{tabular}

In these impingement jet numerical study, two working fluids has been used. One is pure water and the other is $\mathrm{Al}_{2} \mathrm{O}_{3}$-water nanofluid with various particle volume fractions $(0.02 \%, 0.05 \%, 0.1 \%$, and $0.15 \%)$. Properties of Material is specified in 
Table 3. Properties of nanofluids has been calculated using some equations from chapter 2, in MATLAB.

\begin{tabular}{|c|c|c|c|c|}
\hline Material & $\rho\left(\mathrm{kg} / \mathrm{m}^{3}\right)$ & $\begin{array}{c}\mathrm{C}_{\mathrm{p}} \\
(\mathrm{J} / \mathrm{kgK})\end{array}$ & $\mu$ (Pas) & $k(W / m K)$ \\
\hline Water & 998 & 4182 & $9.9800 \mathrm{e}-04$ & 0.5970 \\
\hline $\begin{array}{c}\text { Nanofluid } \\
\mathrm{Al}_{2} \mathrm{O}_{3}-\mathrm{H}_{2} \mathrm{O} \\
(\mathbf{0 . 0 2} \%)\end{array}$ & $1.0556 \mathrm{e}+03$ & $3.9314 \mathrm{e}+03$ & 0.0010 & 0.6318 \\
\hline $\begin{array}{c}\text { Nanofluid } \\
\mathrm{Al}_{2} \mathrm{O}_{3}-\mathrm{H}_{2} \mathrm{O} \\
(\mathbf{0 . 0 5} \%)\end{array}$ & $1.1421 \mathrm{e}+03$ & $3.6029 \mathrm{e}+03$ & 0.0011 & 0.6865 \\
\hline $\begin{array}{c}\text { Nanofluid } \\
\mathrm{Al}_{2} \mathrm{O}_{3}-\mathrm{H}_{2} \mathrm{O} \\
(0.10 \%)\end{array}$ & $1.2862 \mathrm{e}+03$ & $3.1536 \mathrm{e}+03$ & 0.0012 & 0.7854 \\
\hline $\begin{array}{c}\text { Nanofluid } \\
\mathrm{Al}_{2} \mathrm{O}_{3}-\mathrm{H}_{2} \mathrm{O} \\
(0.15 \%)\end{array}$ & $1.4303 e+03$ & $2.7949 \mathrm{e}+03$ & 0.0014 & 0.8953 \\
\hline $\begin{array}{c}\text { Inconel } \\
\text { 601GC } \\
\text { Plate } \\
\end{array}$ & 8110.2121 & 448 & - & 11 \\
\hline
\end{tabular}

For the 2D Planer model simulation was done with constant heat flux boundary condition at the Inconel Plate, consuming $720 \mathrm{~W}$, with various inlet fluid speeds, according to the Reynolds number, and inlet fluid temperature of $15^{\circ} \mathrm{C}$. Table 4 shows boundary conditions inlet speed, which was calculated using equations from chapter 2, in MATLAB. 


\begin{tabular}{|c|c|c|c|c|c|}
\hline $\begin{array}{c}\mathbf{\varnothing} \mathbf{~ \%} \\
\left(\mathbf{A l}_{2} \mathbf{O}_{3}\right)\end{array}$ & $\mathbf{R e}=\mathbf{1 7 3 2}$ & $\mathbf{R e}=\mathbf{2 0 0 0}$ & $\mathbf{R e}=\mathbf{2 2 6 1}$ & $\mathbf{R e}=\mathbf{2 5 0 0}$ & $\mathbf{R e}=\mathbf{2 7 1 9}$ \\
\hline $\mathbf{0 . 0 0}$ (Water) & 1.1320 & 1.3070 & 1.4780 & 1.6340 & 1.7770 \\
\hline $\mathbf{0 . 0 2}$ & 1.1237 & 1.2976 & 1.4669 & 1.6220 & 1.7641 \\
\hline $\mathbf{0 . 0 5}$ & 1.1128 & 1.2850 & 1.4527 & 1.6063 & 1.7470 \\
\hline $\mathbf{0 . 1 0}$ & 1.0980 & 1.2679 & 1.4333 & 1.5848 & 1.7237 \\
\hline $\mathbf{0 . 1 5}$ & 1.0861 & 1.2541 & 1.4178 & 1.5677 & 1.7050 \\
\hline
\end{tabular}

Table 4 Inlet speed $(\mathrm{m} / \mathrm{s})$

All the 2D planer simulations were done using Pressure-based steady solver in ANSYS FLUENT. Heat transfer properties: the heat transfer coefficient, Plate temperature and the Nusselt number were calculated for different cases. In all the simulations, the jet diameter $\mathrm{d}=1.53 \mathrm{~mm}$ and the distance from nozzle to wall surface $\mathrm{z}=10 \mathrm{~mm}$ were held constant. 


\section{CHAPTER 4}

\subsection{Results}

In this chapter, the results for the 2D-planer model are presented. Results are compared with the experimental results [9] from the literature. For this numerical analysis, two working fluids are used, pure water and the $\mathrm{Al}_{2} \mathrm{O}_{3}$-water nanofluid with various particle volume fractions $(0.02 \%, 0.05 \%, 0.1 \%$, and $0.15 \%)$, with various Reynolds numbers (1732, 2000, 2261, 2500, and 2719). Due to symmetric geometry one side results, of a targeted Inconel plate, has been plotted. For each case, the local heat transfer coefficient, h, Plate temperature $\mathrm{T}$, on the target plate and the average heat transfer coefficient, $\sim \mathrm{h}$, has been calculated.

\subsubsection{Plate temperature for Water \& Nanofluid}

Surface temperature distribution of the one side of the target plate for water has been shown in Figure 15, and for nanofluid with particle volume fractions of $0.02 \%$ (Figure 16), 0.05\% (Figure 17), 0.1\% (Figure 18), and 0.15\% (Figure 19) at various Reynolds numbers. For each case, the local heat transfer coefficient, h, on the target plate at specified points along the fluid flow and the average heat transfer coefficient, $\overline{h_{\text {num }}}$, has been recorded. For detailed plots see Appendix A.

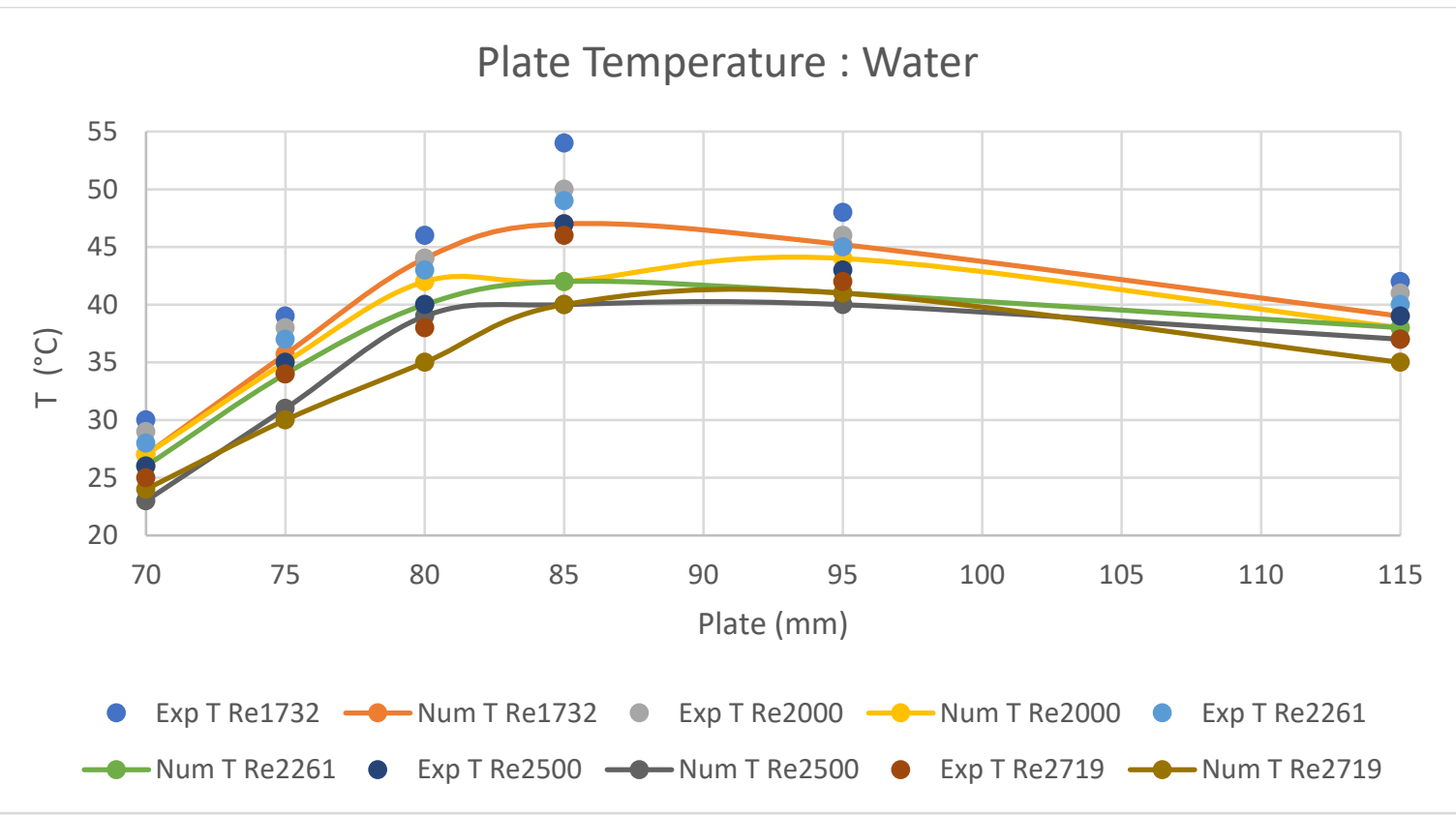

Figure 17 Plate temperature distribution of the one side of the target plate for water 


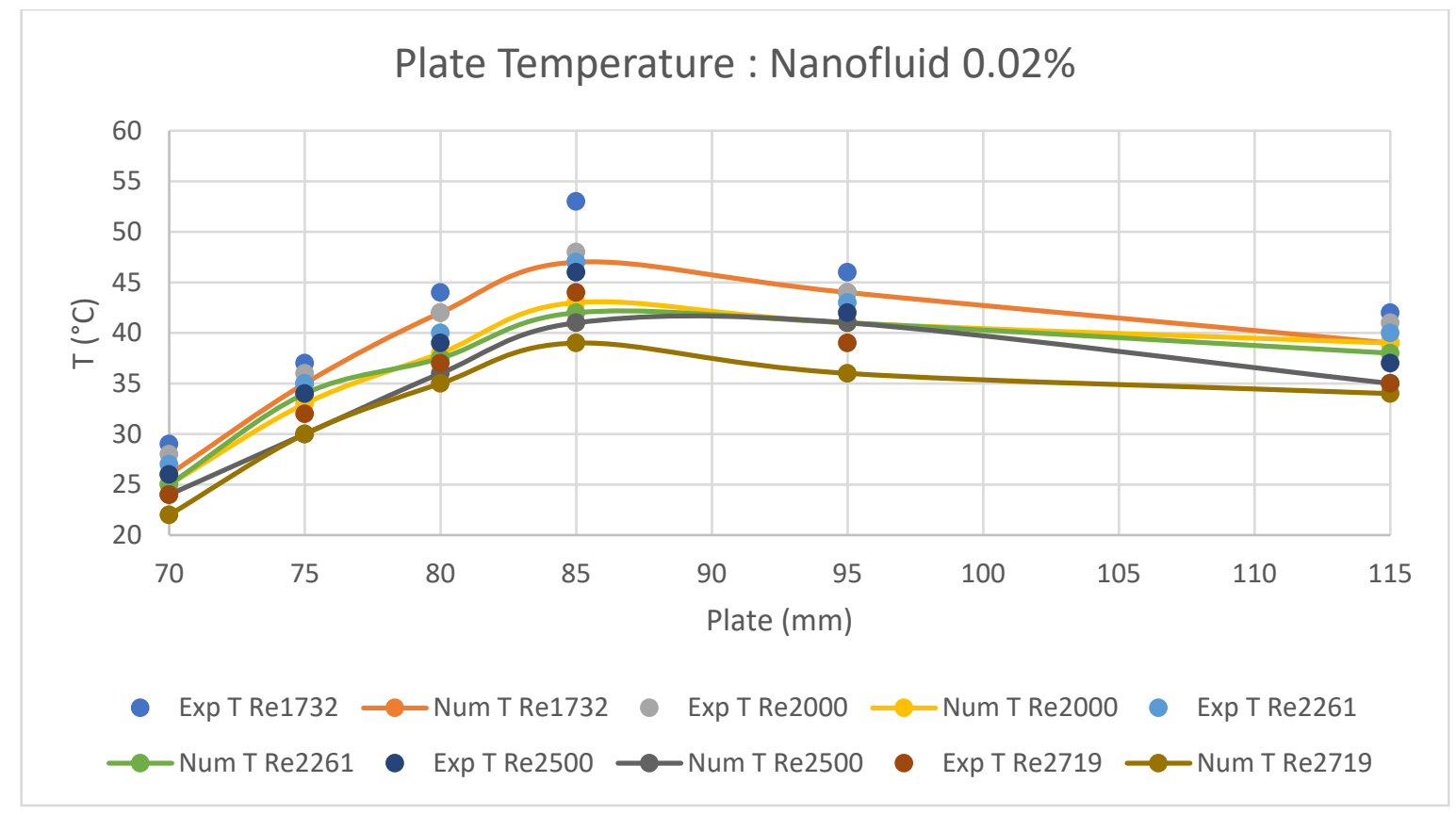

Figure 18 Plate temperature distribution of the one side of the target plate for $\mathrm{Al}_{2} \mathrm{O}_{3}$-water nanofluid with particle volume fraction of $0.02 \%$

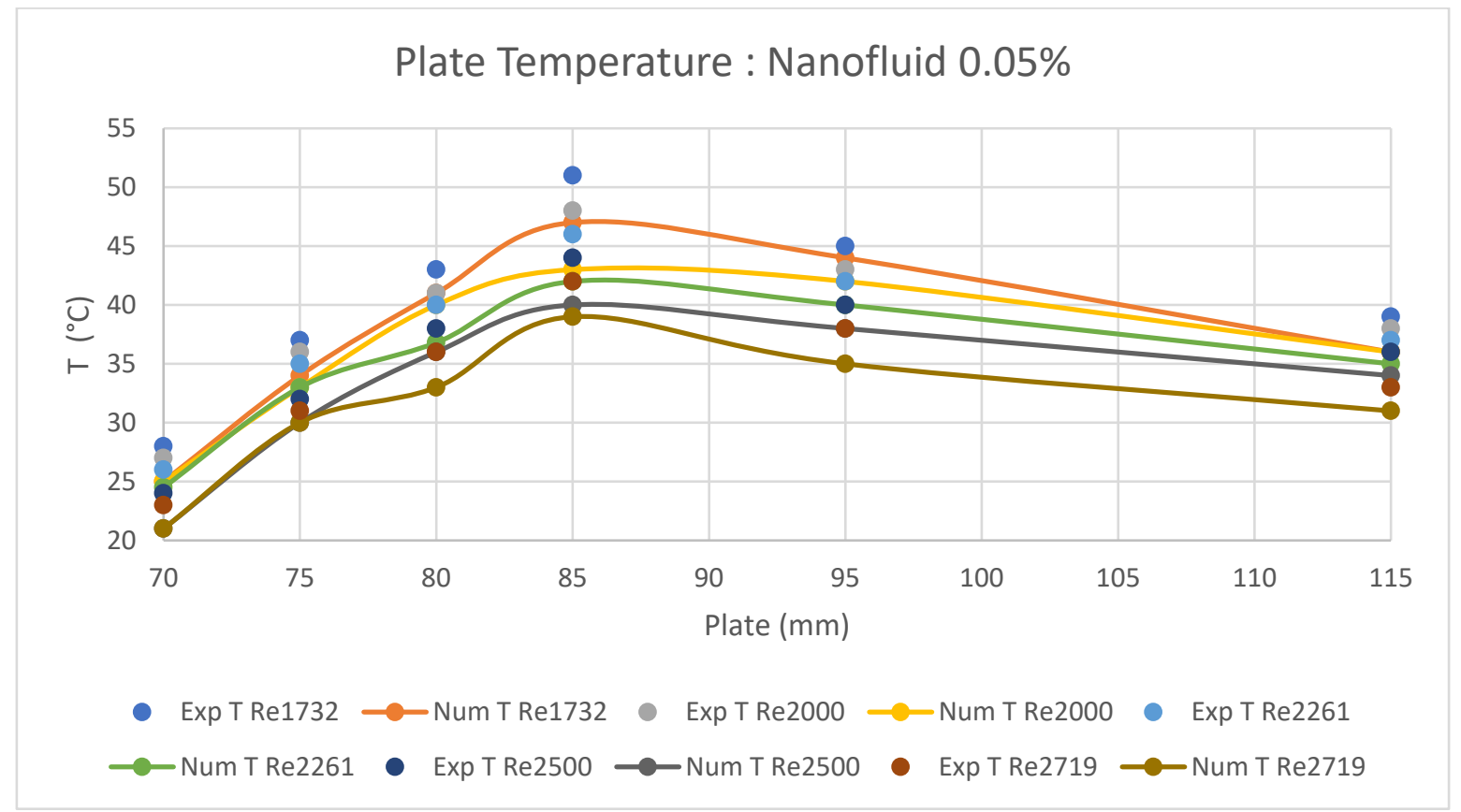

Figure 19 Plate temperature distribution of the one side of the target plate for $\mathrm{Al}_{2} \mathrm{O}_{3}$-water nanofluid with particle volume fraction of $0.05 \%$ 


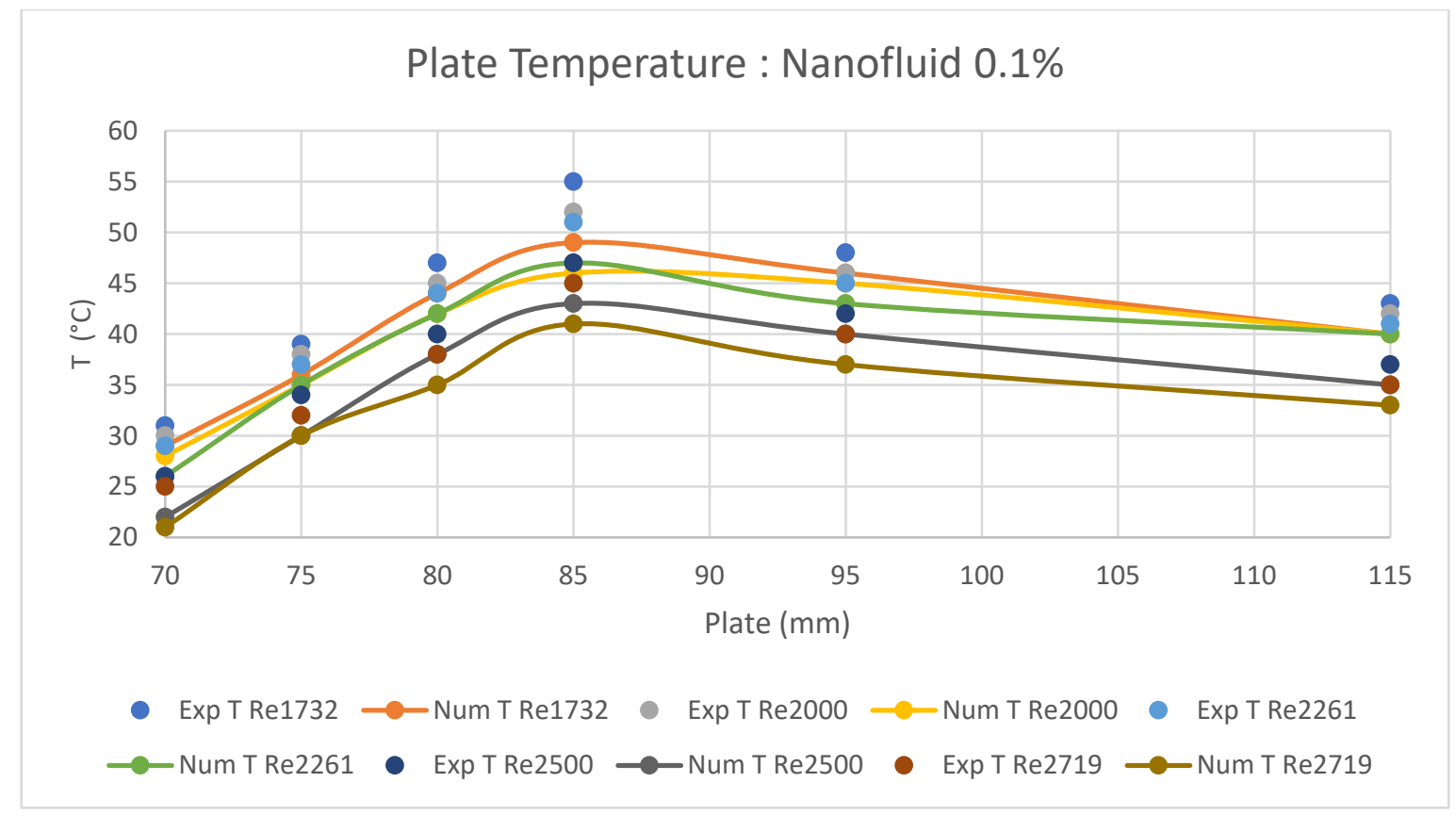

Figure 20 Plate temperature distribution of the one side of the target plate for $\mathrm{Al}_{2} \mathrm{O}_{3}$-water nanofluid with particle volume fraction of $0.10 \%$

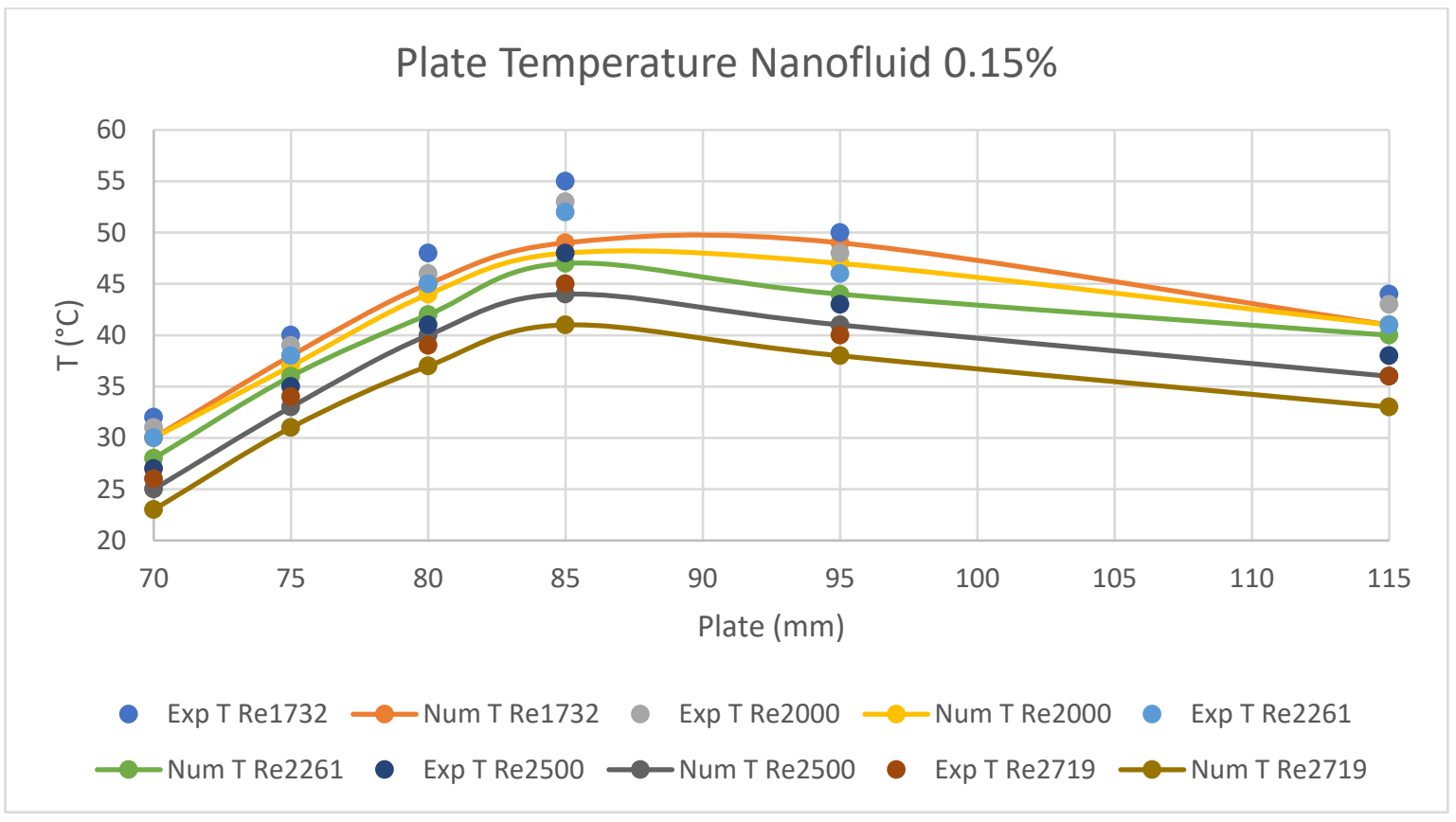

Figure 21 Plate temperature distribution of the one side of the target plate for $\mathrm{Al}_{2} \mathrm{O}_{3}$-water nanofluid with particle volume fraction of $0.15 \%$ 


\subsubsection{Local heat transfer coefficients of water}

From Figure 21, it can be seen, that the local heat transfer coefficient is maximum at the stagnation point $(\mathrm{X}=70 \mathrm{~mm})$. Boundary layer thickness is minimum at this point, promoting maximum heat transfer, and maximum cooling. The local heat transfer coefficient tended to decrease for increasing $\mathrm{x}$, because of growth of boundary layer. This figure also indicates that the local heat transfer coefficient increases after a dramatic decrease. As the fluid moved down the V-shape plate, the fluid was accelerated due to the influence of gravity. Due gravity effect fluid velocities increase after points, $X=85 \mathrm{~mm}$. Local heat transfer coefficients increase with increasing Reynolds number, due to increase in velocity. From below plot, we can observe that, at stagnation point $(\mathrm{X}=70 \mathrm{~mm}$ ), Re 2719 has highest $\mathrm{h}$ and $\mathrm{Re}$ 1732 has lowest value of $\mathrm{h}$. Increase in the heat transfer coefficient is about $50 \%$, which is higher than that for other points. Variation between numerical study results and experimental results are about $\pm 6 \%$ to $\pm 9 \%$, which is acceptable. For detailed plots see Appendix A and Appendix B.

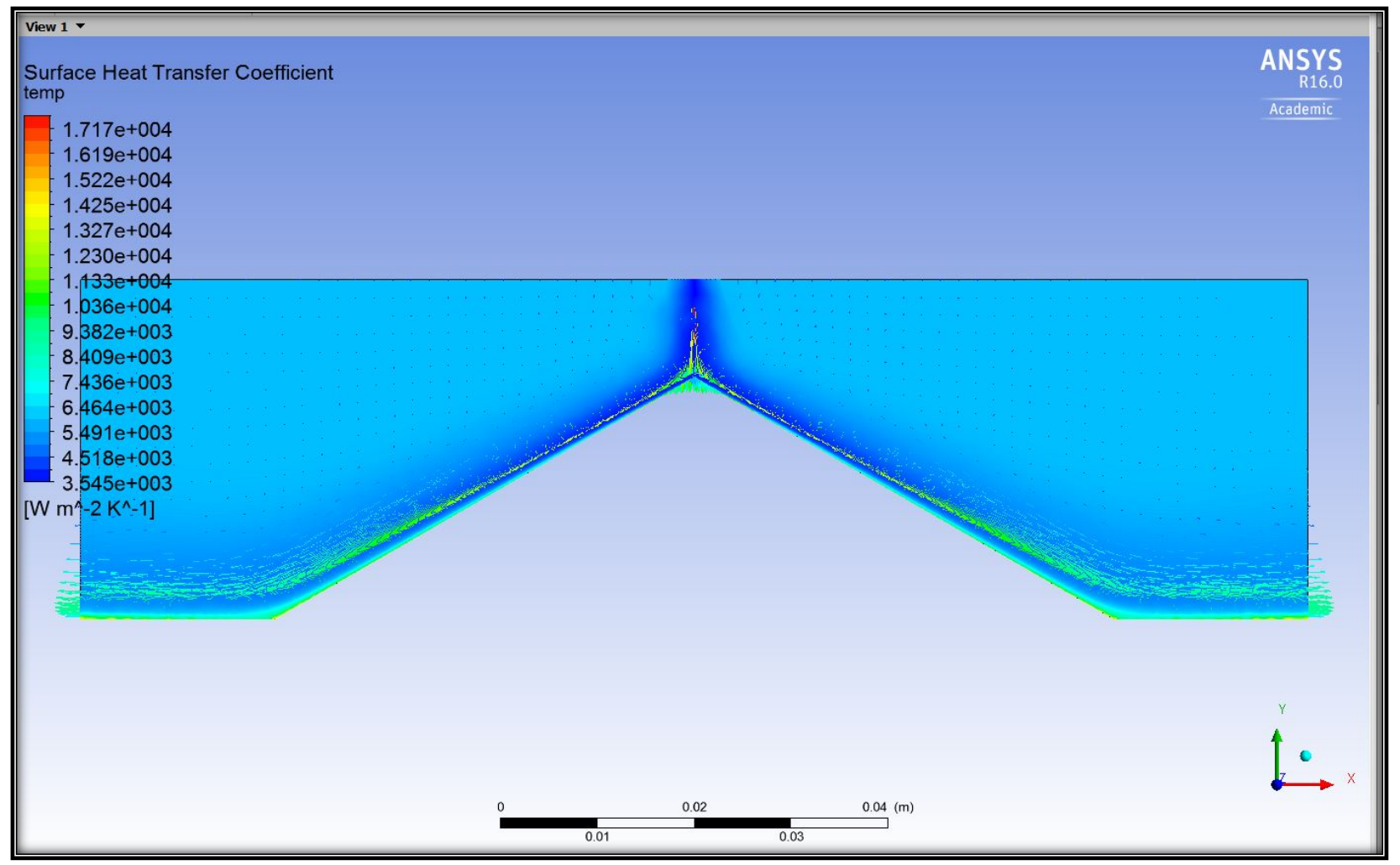

Figure 22 ANSYS CFD Post Surface heat transfer coefficient output: Water at Re 2719 


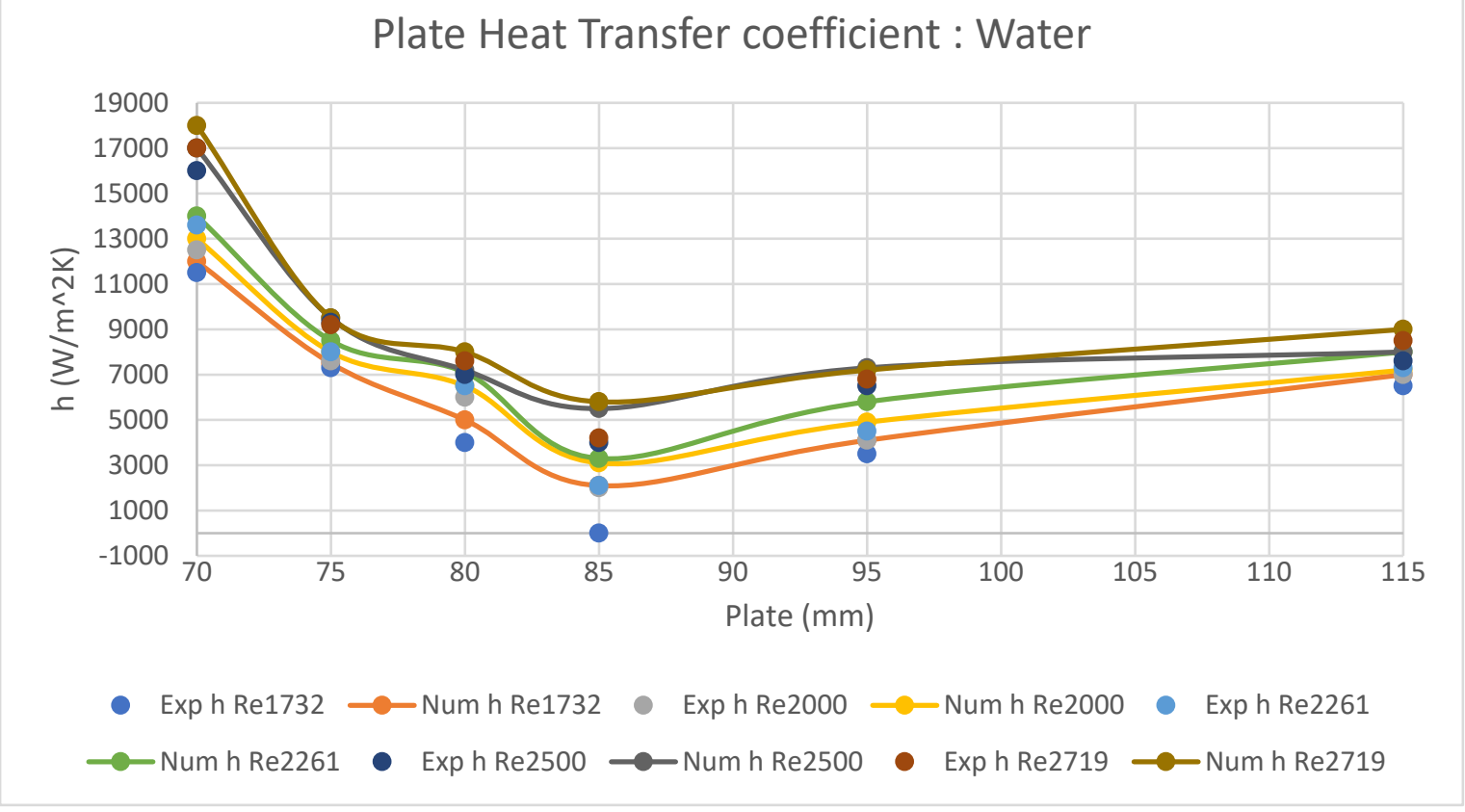

Figure 23 Distribution of the local impinging jet heat transfer coefficient with the use of water.

\subsubsection{Local heat transfer coefficients of $\mathrm{Al}_{2} \mathrm{O}_{3}$-water nanofluid}

Same trend, like water plot for local heat transfer coefficient, has been recorded for Nanofluid, despite the difference in the magnitude of the local heat transfer coefficients, the local heat transfer coefficients increase with increasing Reynolds number, and maximum local heat transfer coefficient at highest Reynolds number 2719, as expected. For $0.02 \%$ nanofluid increase in h w.r.t. Re is about $47.69 \%$. For $0.05 \%, 0.10 \%$ and $0.15 \%$ nanofluid are $49.29 \%, 46.36 \%$, 50.94\% respectively. For nanoparticle concentrations lower than $0.05 \%$ the local heat transfer coefficients are increased with increasing nanoparticle volume concentration. However, for nanoparticle concentrations higher than $0.05 \%$ it can be seen clearly that the local heat transfer coefficients are decreased with increasing nanoparticle volume concentration. Maximum range is obtained at $0.05 \%$ concentration nanofluid. Variation between numerical study results and experimental results are about $\pm 6 \%$ to $\pm 9 \%$, which is acceptable. For detailed plots see Appendix A and Appendix B. 


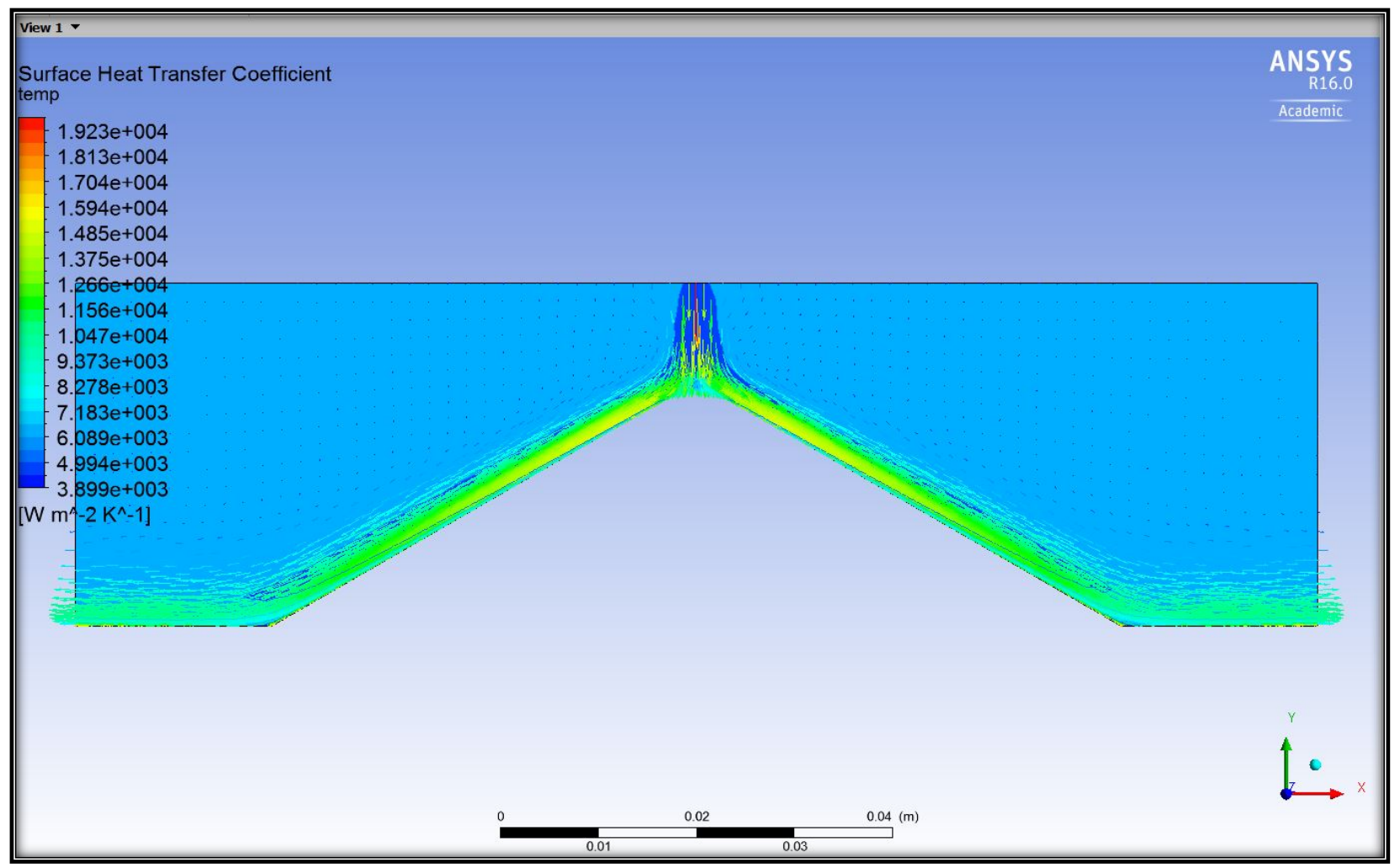

Figure 24 ANSYS CFD Post Surface heat transfer coefficient output: Nanofluid 0.05\% at Re 2719

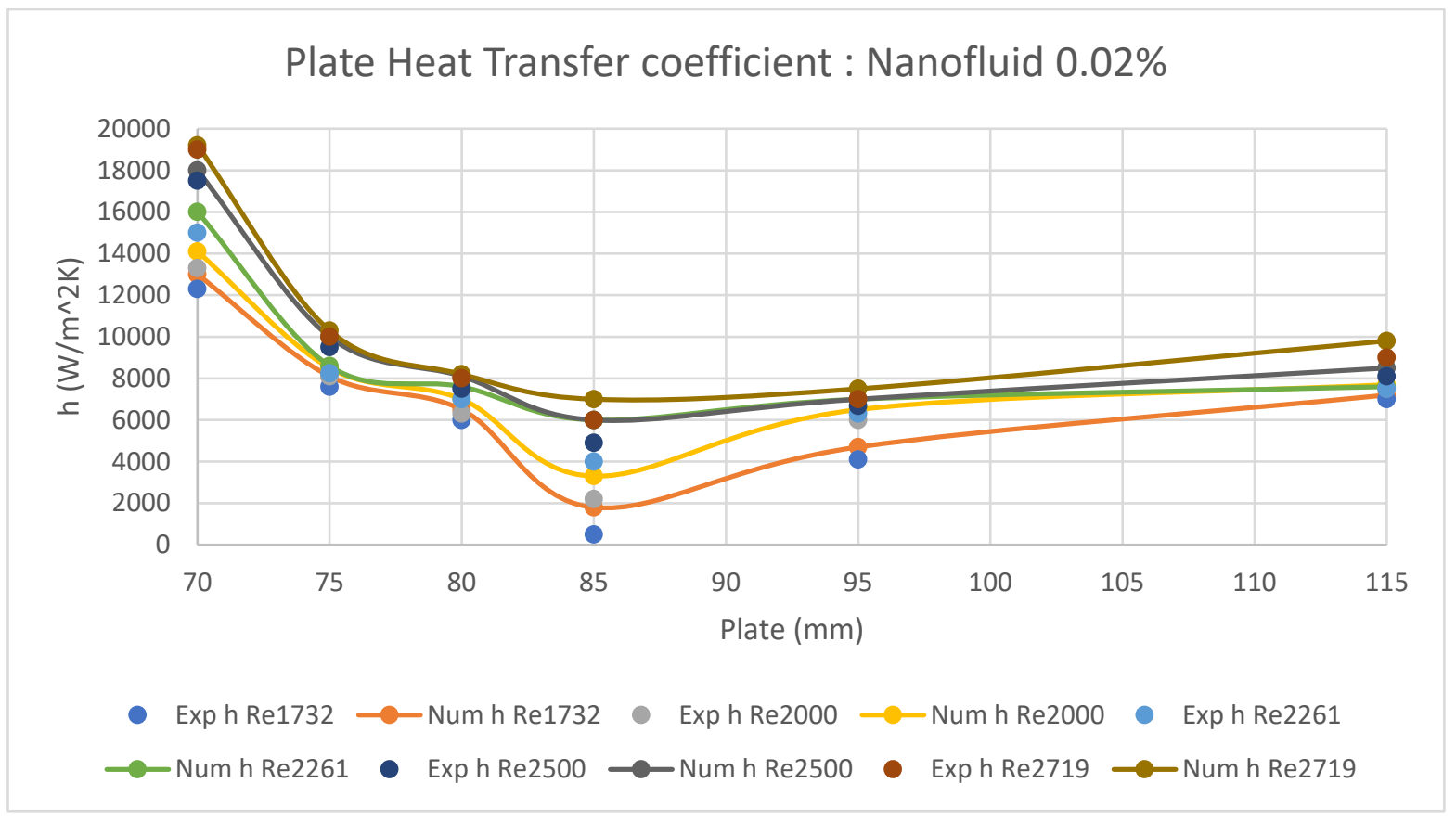

Figure 25 Distribution of the local impinging jet heat transfer coefficient with the use of $\mathrm{Al}_{2} \mathrm{O}_{3}$-water nanofluid with particle volume fraction of $0.02 \%$ 


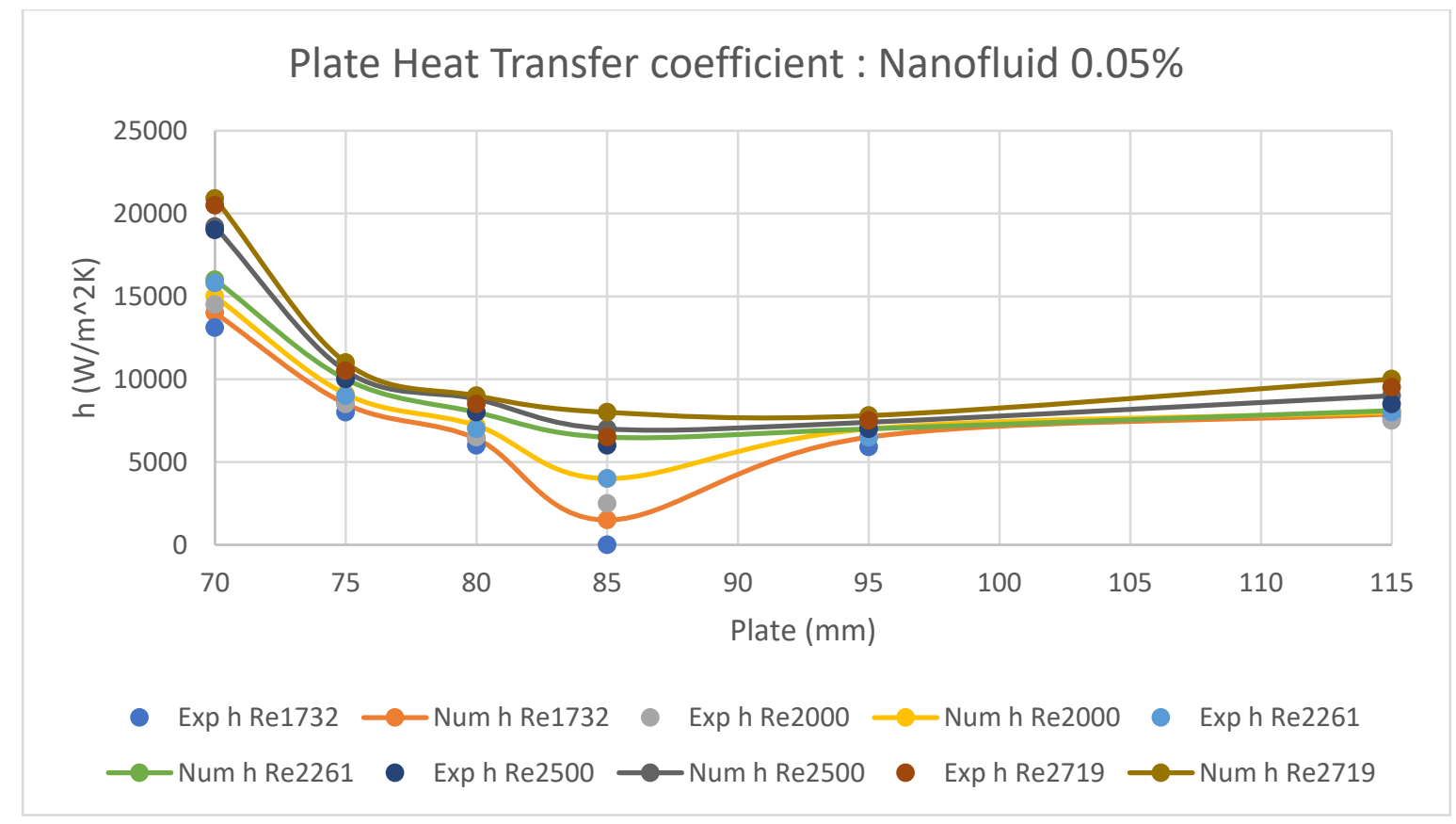

Figure 26 Distribution of the local impinging jet heat transfer coefficient with the use of $\mathrm{Al}_{2} \mathrm{O}_{3}$-water nanofluid with particle volume fraction of $0.05 \%$

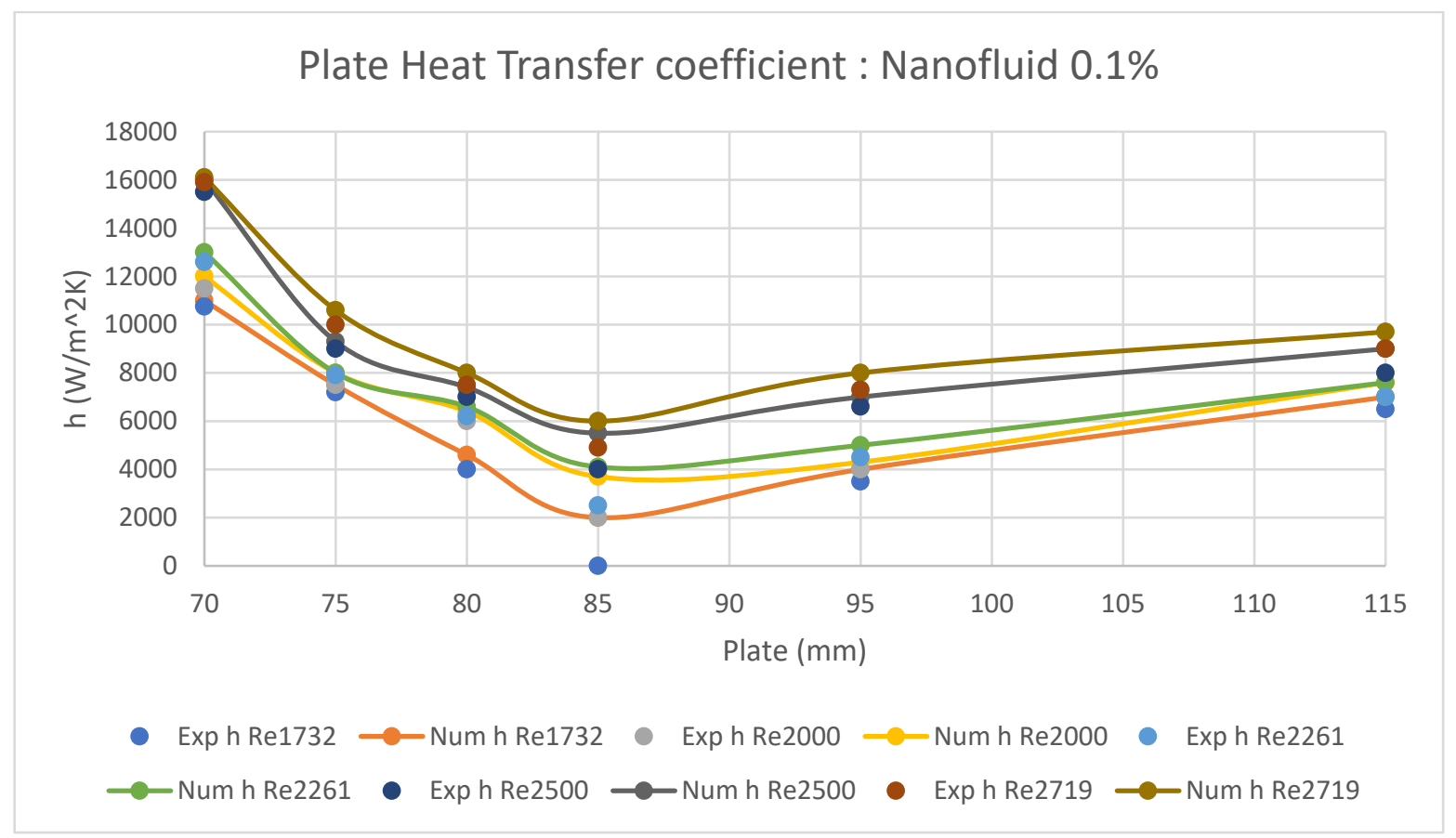

Figure 27 Distribution of the local impinging jet heat transfer coefficient with the use of $\mathrm{Al}_{2} \mathrm{O}_{3}$-water nanofluid with particle volume fraction of $0.10 \%$ 


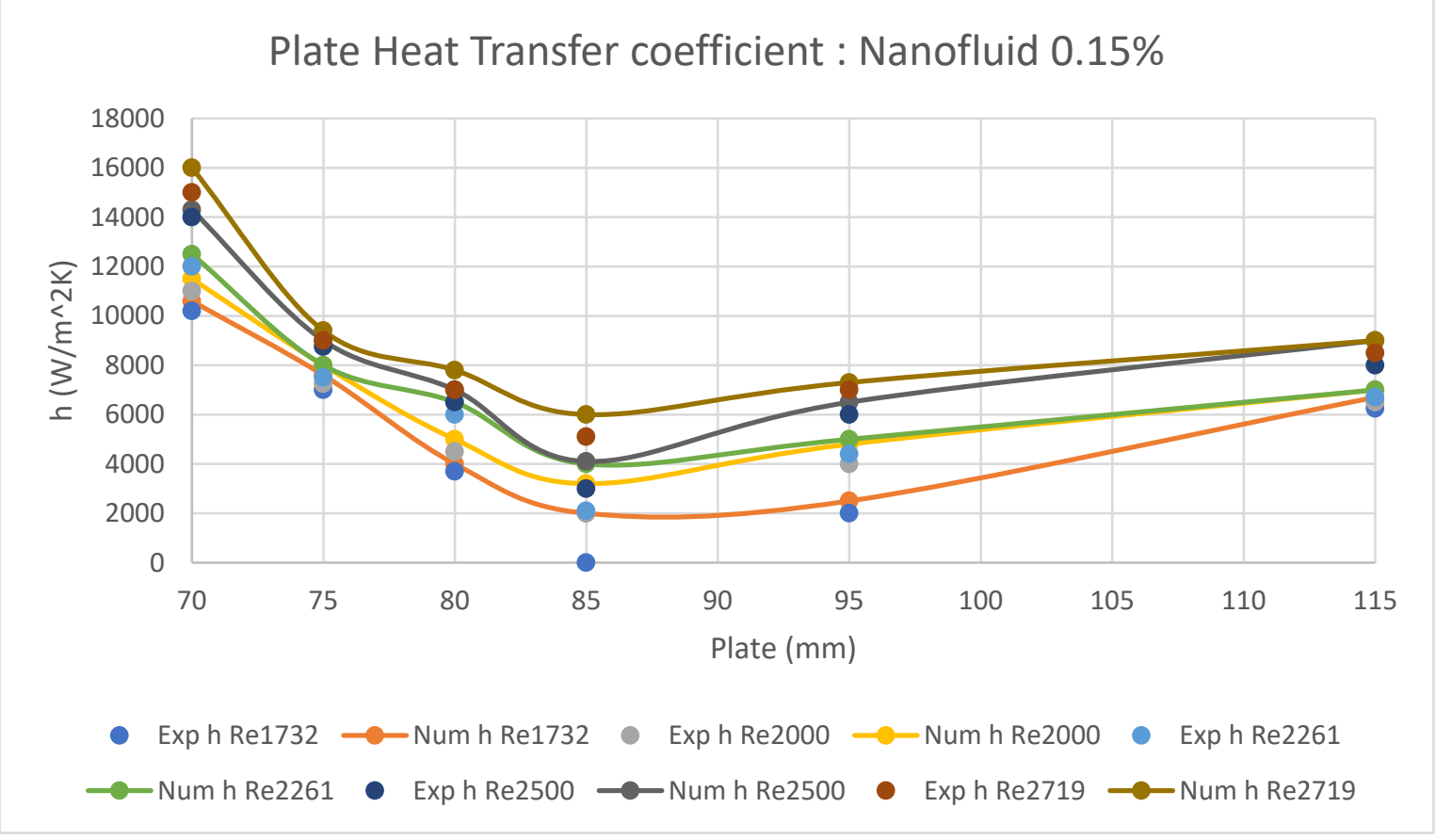

Figure 28 Distribution of the local impinging jet heat transfer coefficient with the use of $\mathrm{Al}_{2} \mathrm{O}_{3}$-water nanofluid with particle volume fraction of $0.15 \%$

Fig. 27-31, represents local heat transfer coefficients w.r.t. nanoparticle volume concentrations at each Reynolds number. From plots, it can be easily observed that maximum heat transfer, and maximum cooling, is taking place at $70 \mathrm{~mm}$ i.e. at stagnation point. Nanoparticles volume concentration plays a significant role in heat transfer coefficient level. As volume concentration increases from $0.02 \%$ to $0.05 \%$

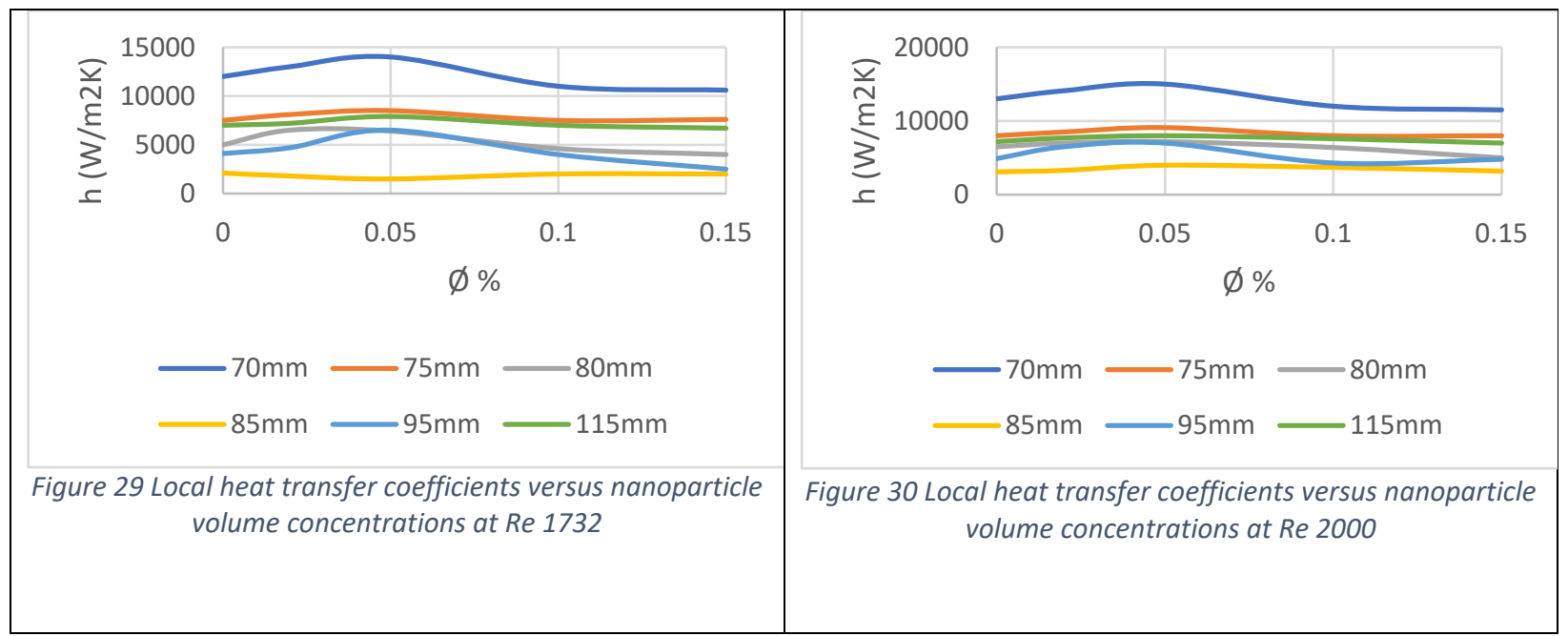




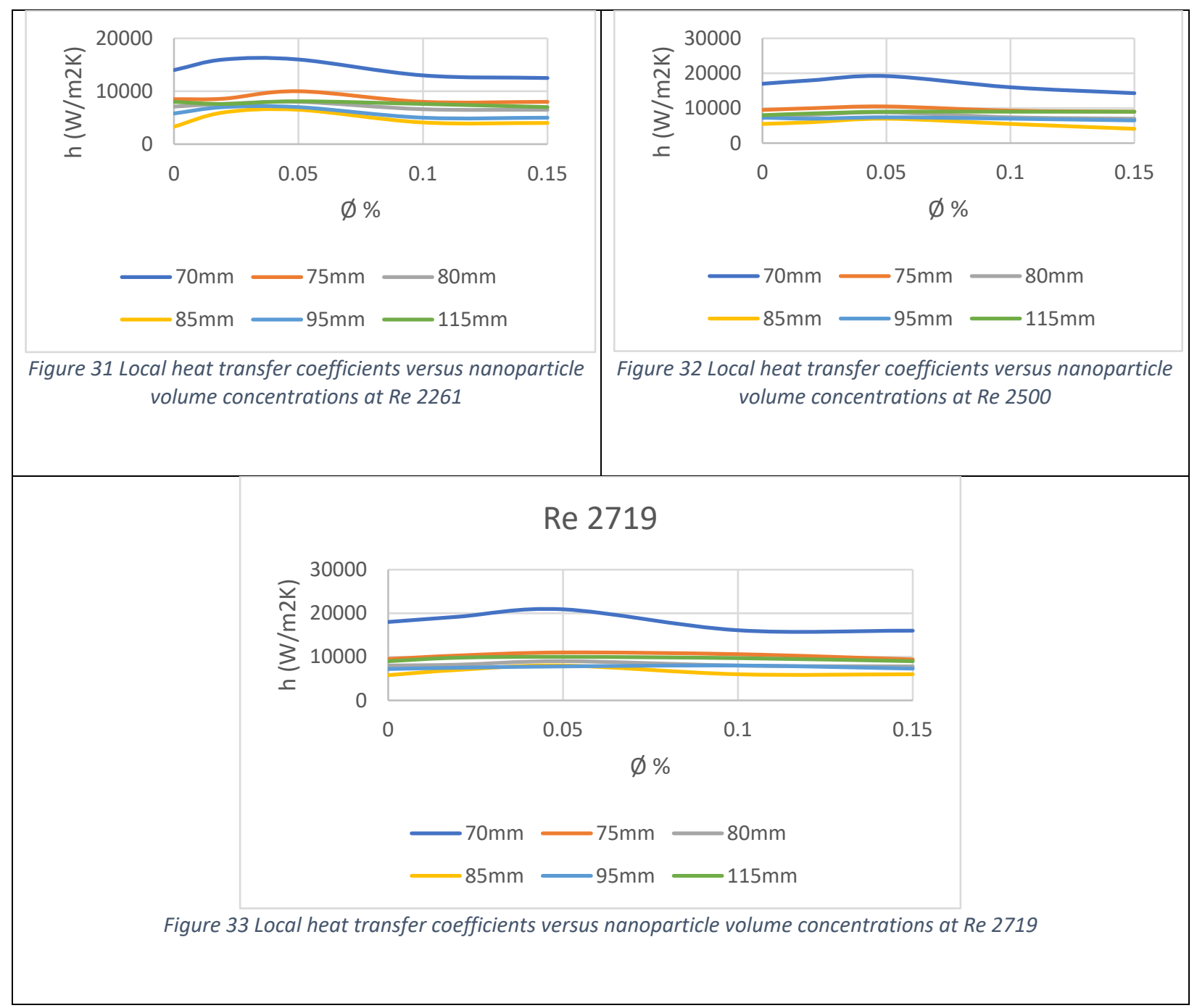

Local heat transfer coefficient increases. But this trend has been reversed for further increase in concentration from $0.05 \%$ to $0.1 \%$ and $0.15 \%$. Maximum local heat transfer coefficient reading has been observed at $0.05 \%$. Figure 32 shows the dimensionless $\left(\mathrm{h}_{\mathrm{nf}} / \mathrm{h}_{\mathrm{w}}\right)$ local heat transfer coefficient ratio w.r.t plate position. At Reynolds numbers 2261and 2719 variation is quite high compared to other Reynolds number values. 


\section{$0.05 \%$}
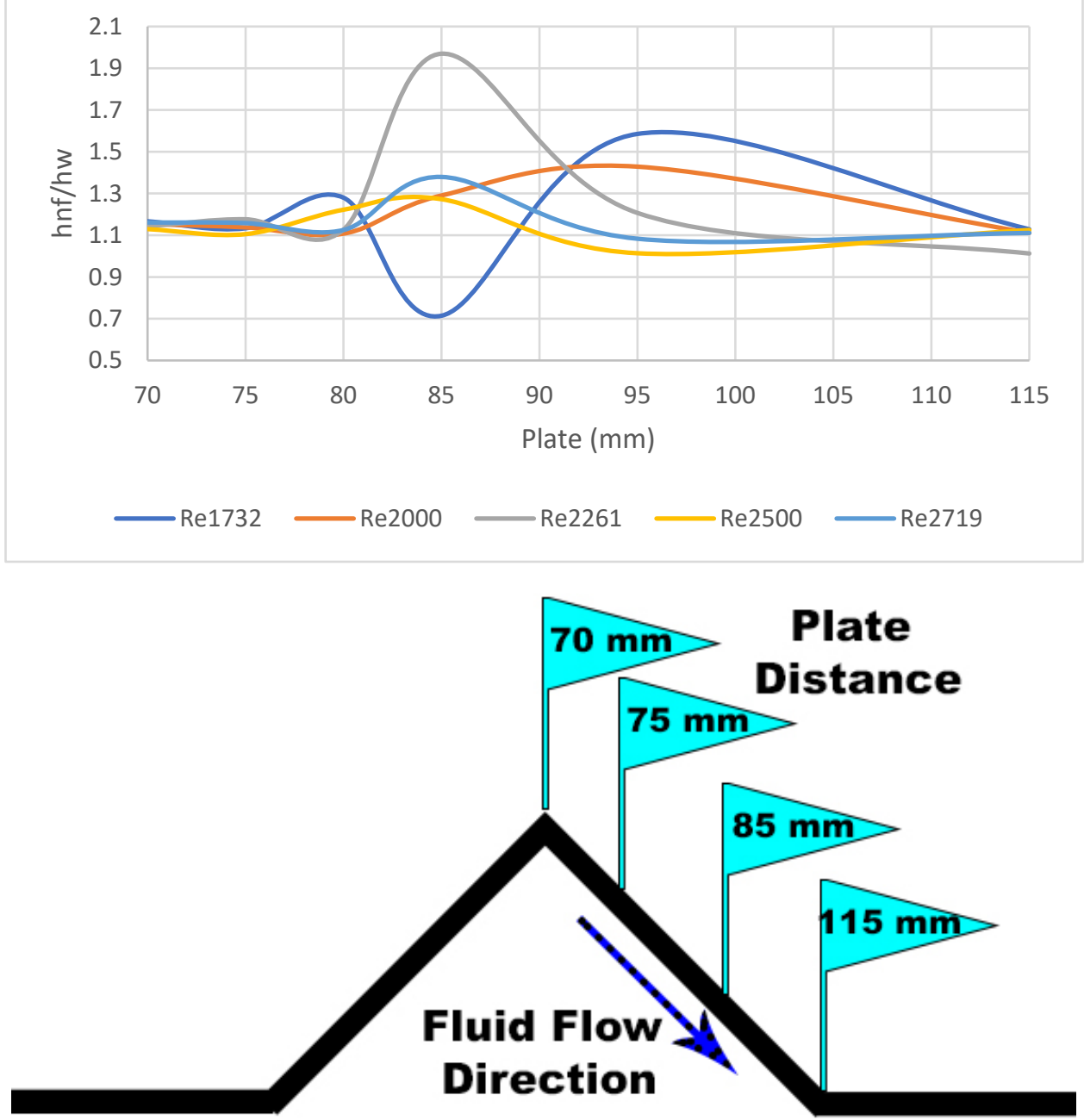

Figure $34 h_{n f} / h_{w}$ Vs Plate Plot

\subsubsection{Average heat transfer coefficients of $\mathrm{Al}_{2} \mathrm{O}_{3}$-water nanofluid}

To observe the effect of the particle volume fraction of nanoparticles on the base fluid heat transfer, average heat transfer coefficient $\left(\overline{h_{\text {num }}}\right)$ to the particle concentration Vs Reynolds number plot was generated, i.e. Figure 33. It can be clearly observed that, $\overline{h_{\text {num }}}$ increases, for all cases, with increase in Reynolds number, as expected. At lower particle concentrations $(0.02 \%$ and $0.05 \%), \overline{h_{\text {num }}}$ clearly increases with increasing particle concentration from $0.02 \%$ to $0.05 \%$, as opposed to higher particle concentrations, $0.1 \%$ and $0.15 \%$. There is a considerable decrease in $\overline{h_{\text {num }}}$ with increasing particle concentration from $0.1 \%$ to $0.15 \%$. 


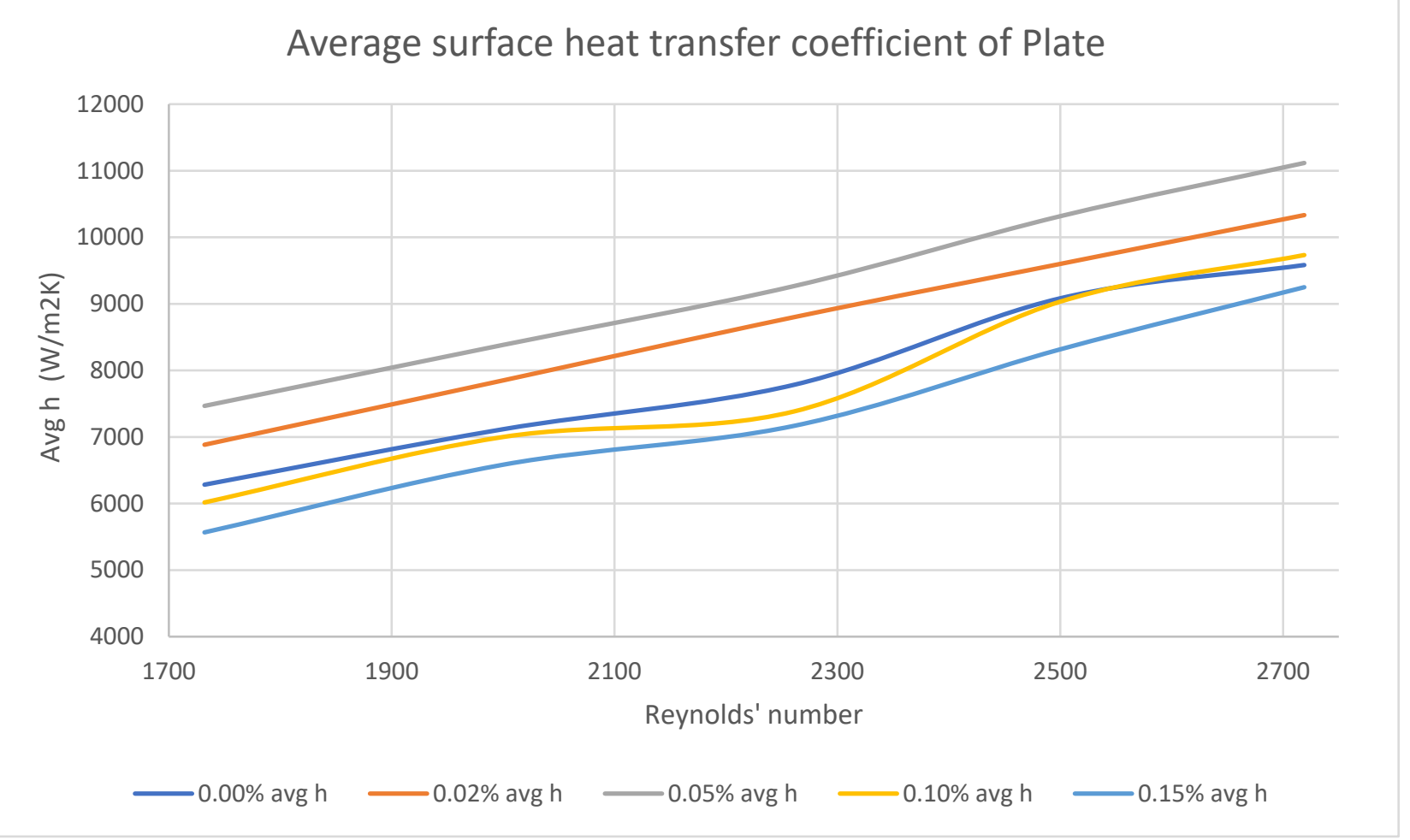

Figure 35 Average Surface heat transfer coefficient for plate ( $\overline{h_{\text {num }}}$ ) Plot w.r.t Reynolds number

\subsection{Conclusion}

Cooling effect of a planar jet impinging vertically on a V-shape plate using water and $\mathrm{Al}_{2} \mathrm{O}_{3}$-water nanofluid is numerically investigated, Using Ansys Workbench 16.0 and results were compared with experimental results [9]. Various cases of different volume fractions nanoparticles and range of Reynolds numbers was studied.

The numerical results show that $\mathrm{Al}_{2} \mathrm{O}_{3}$-water nanofluid with lower concentrations, $0.02 \%$ and $0.05 \%$, yield positive effects on the local heat transfer coefficients and these positive effects increase with increasing Reynolds number as well as volume fraction. For these two concentrations, the maximum increase in the local heat transfer coefficients are about $7 \%$ and $17 \%$ respectively, when compared with water. For nanofluid at higher volume fractions of $0.1 \%$ and $0.15 \%$, negative effects on the local heat transfer coefficients have been observed. At low Reynolds numbers, these negative effects are shown to be more significant than with high Reynolds number. However, at high Reynolds numbers these negative effects appear to have decreased 
and the nanofluid has had a demonstrated ability in increasing the heat transfer coefficient. Finally, the average heat transfer coefficient has indicated a similar trend to the local heat transfer coefficients and the maximum increase of about $20 \%$ has been observed.

Numerical study results are very much in agreement with the experimental results, with variation factor of $\pm 5 \%$ to $\pm 9 \%$, which is quite acceptable. Reasons for variation includes no consideration of nozzle wall thickness in numerical study. Also Cohesion and Adhesion forces for water and nanoparticles plays a vital role in real world, might have lost some accent in numerical studies.

Nanoparticle distribution configuration in the nanofluid volume at any point in time is a key factor, which affects the heat transfer properties of the nanofluid. At high Reynolds number, the nanoparticles are aligned and therefore there is little interaction between them, which would result in poor thermal conductivity. On the other hand, at low Reynolds number, nanoparticle motion is expected to be restricted to assist with the transfer of energy throughout nanofluid volume. Therefore, at optimum Reynolds number and optimum volume fraction concentration, fluid thermal properties, can be improved as observed in this study. 


\section{APPENDIX A}

WATER

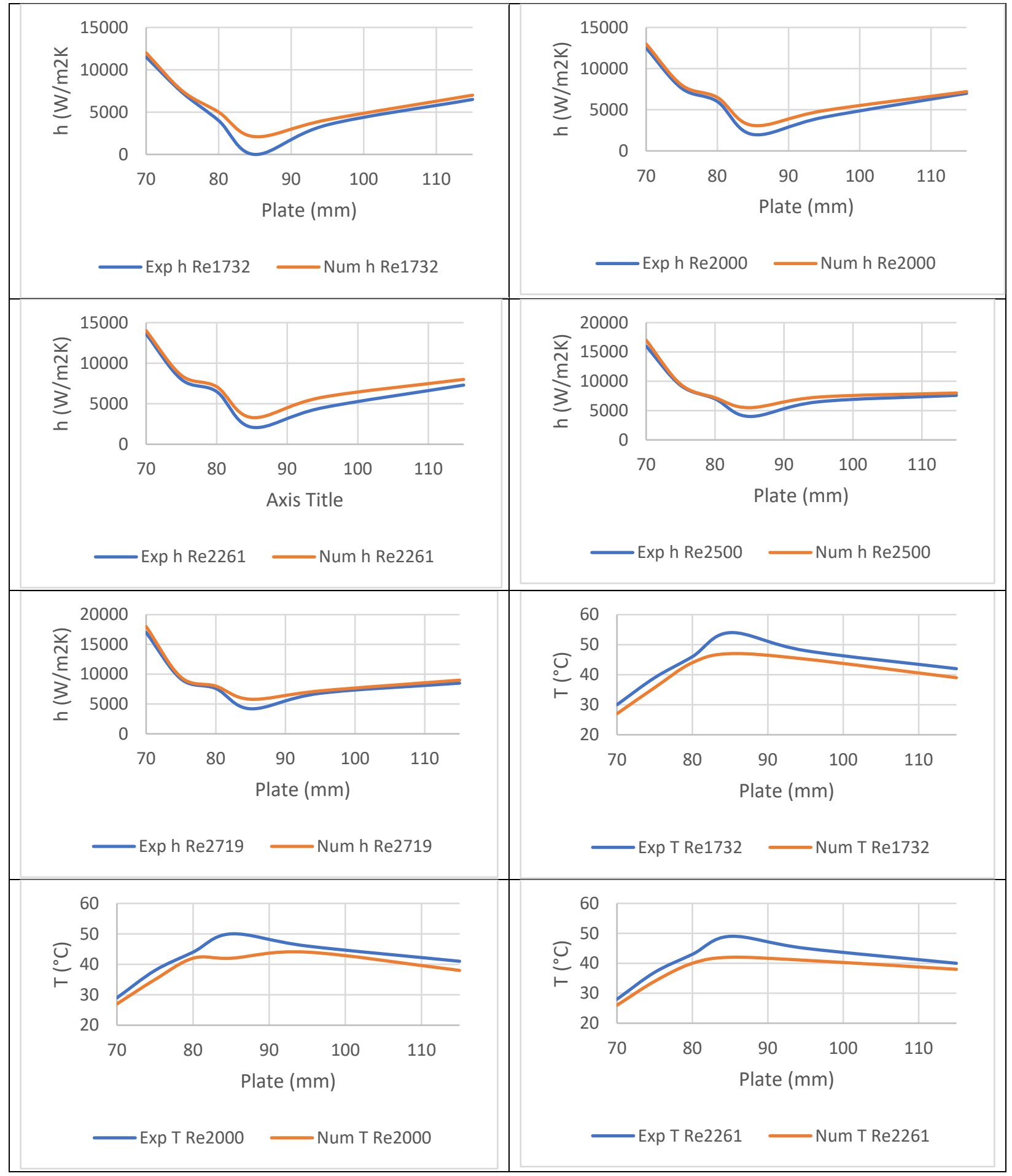




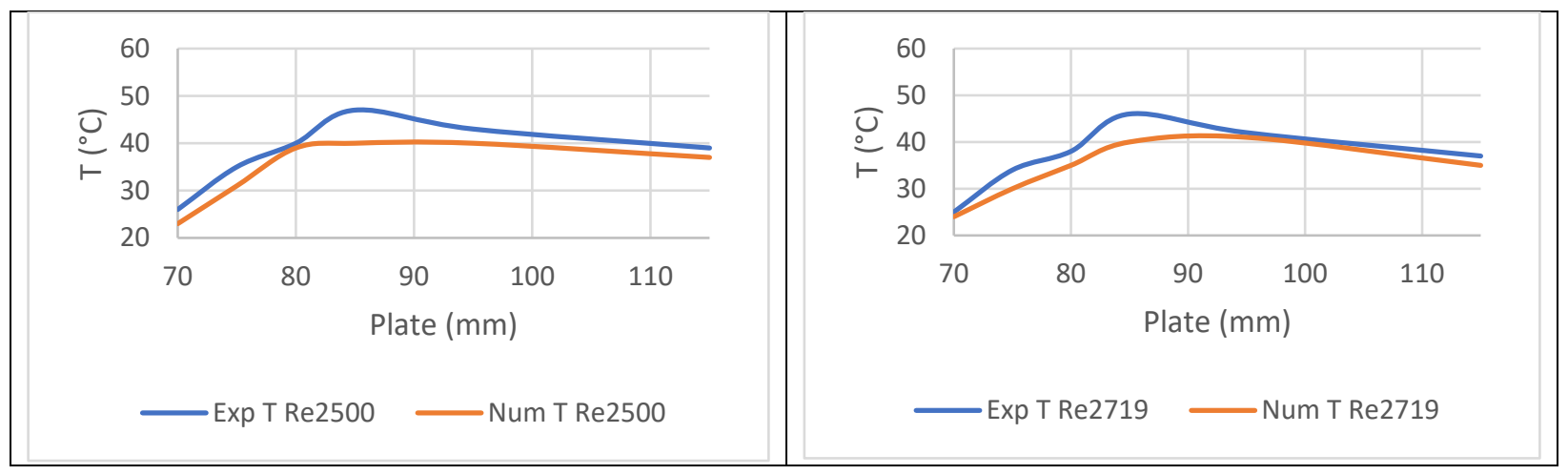

\section{NANOFLUID $0.02 \%$}

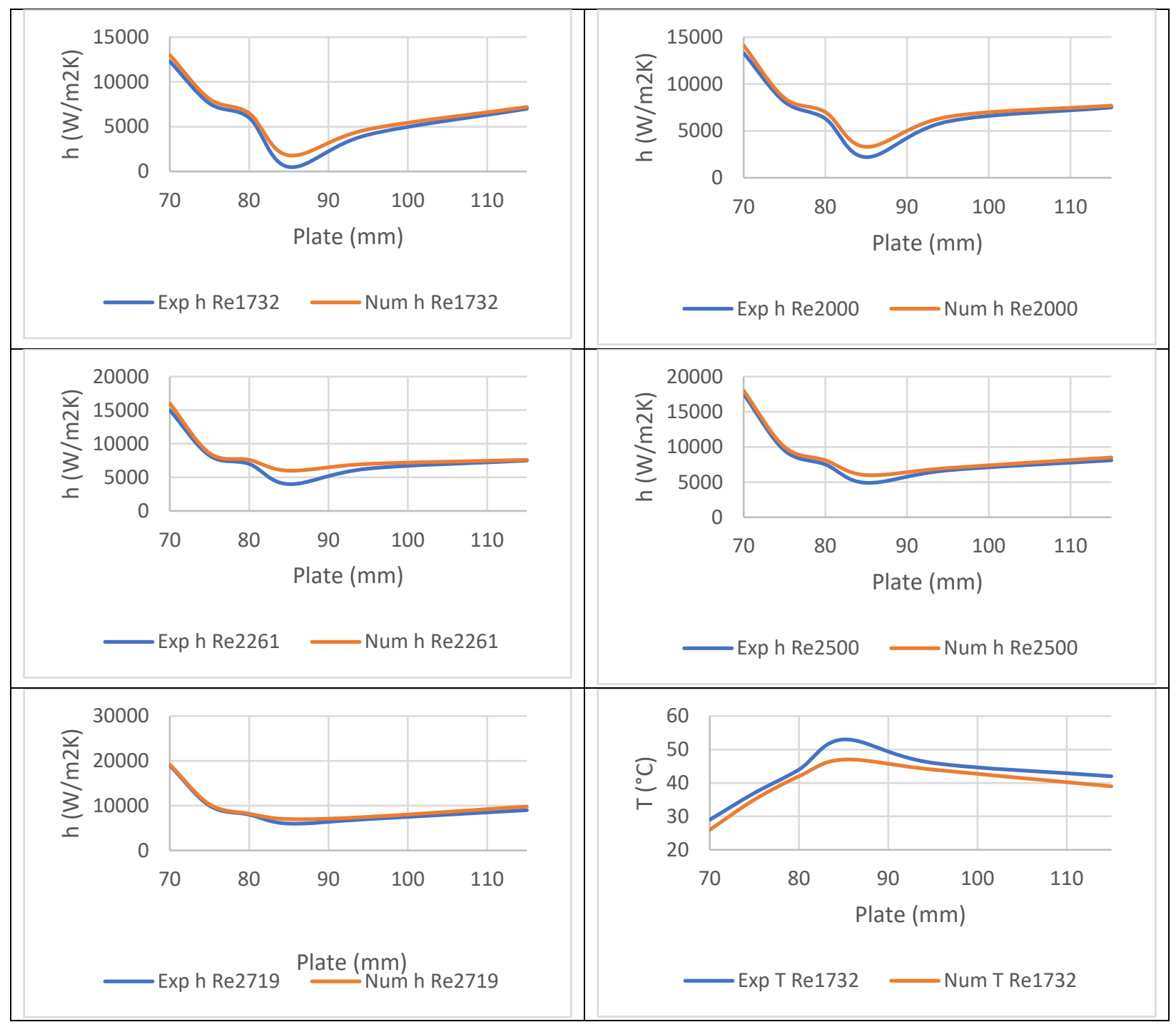




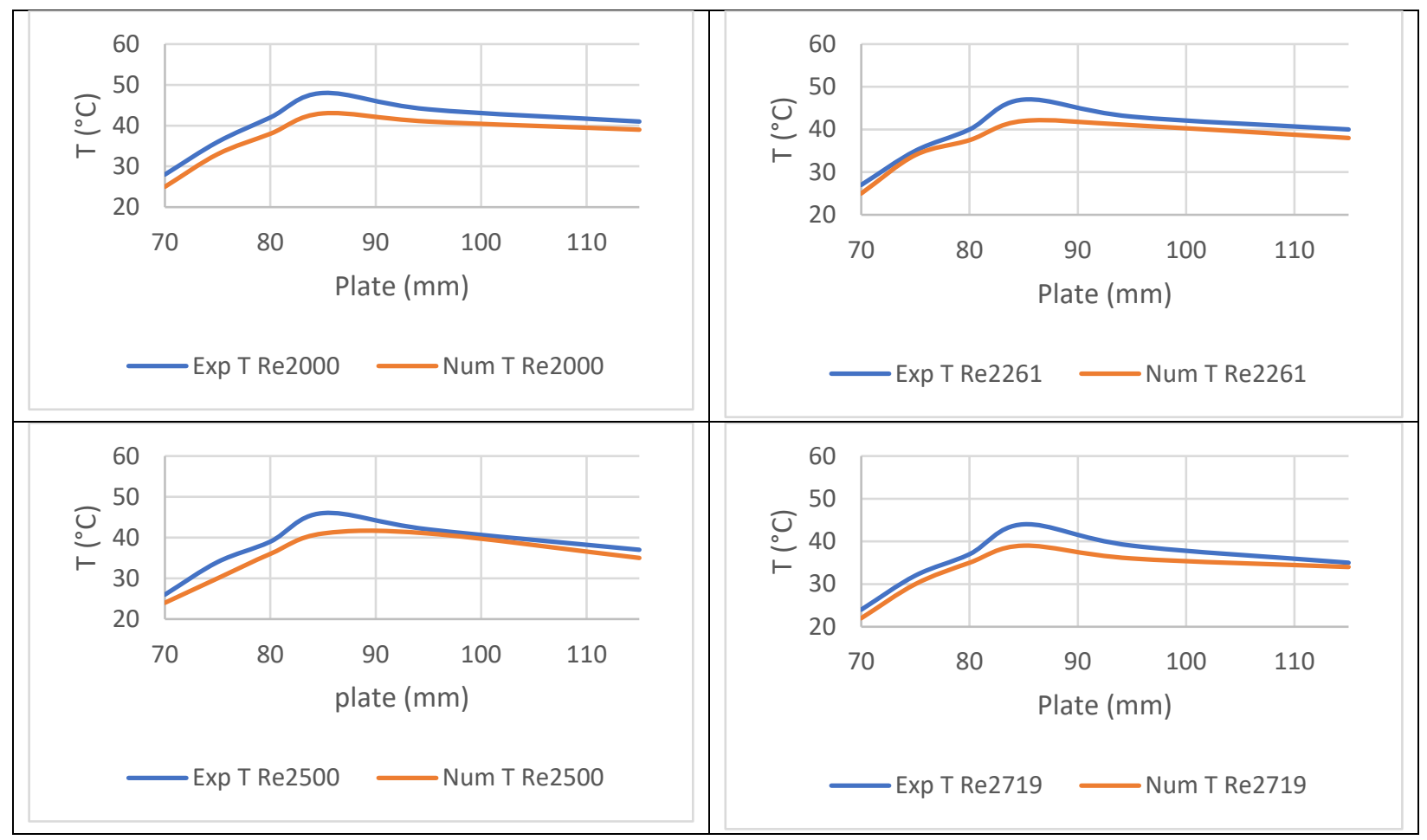

NANOFLUID 0.05\%

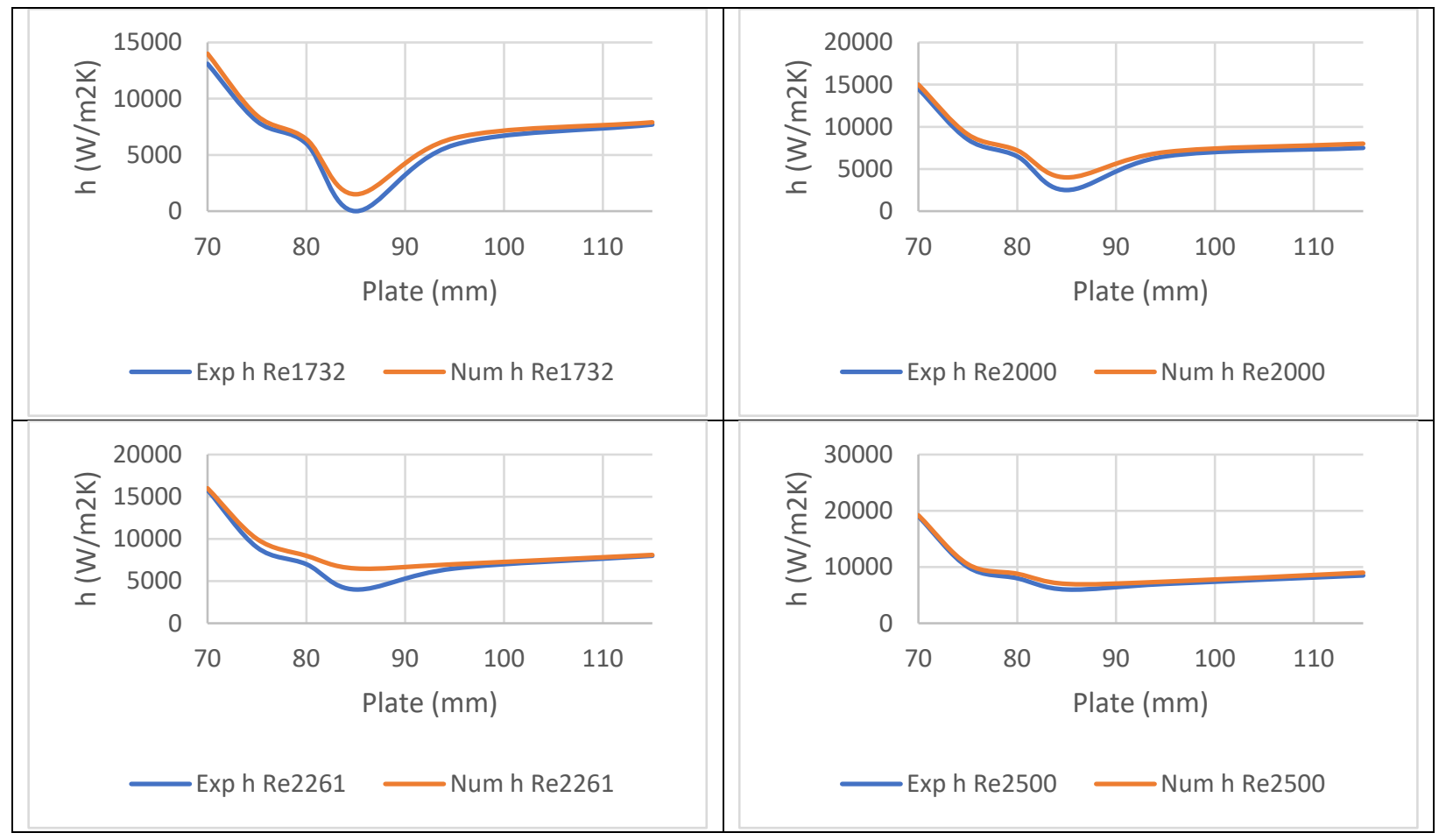




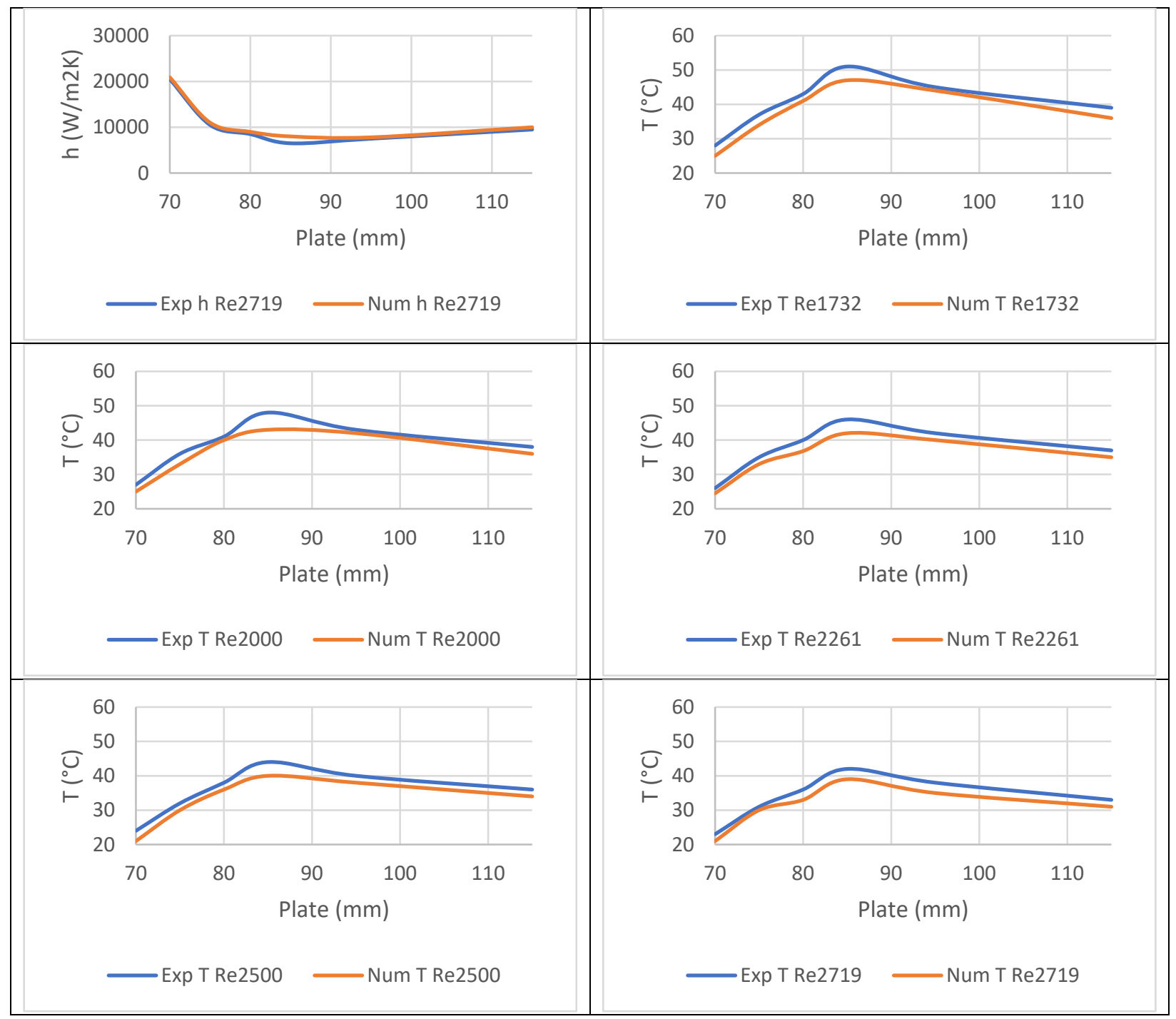




\section{NANOFLUID $0.10 \%$}

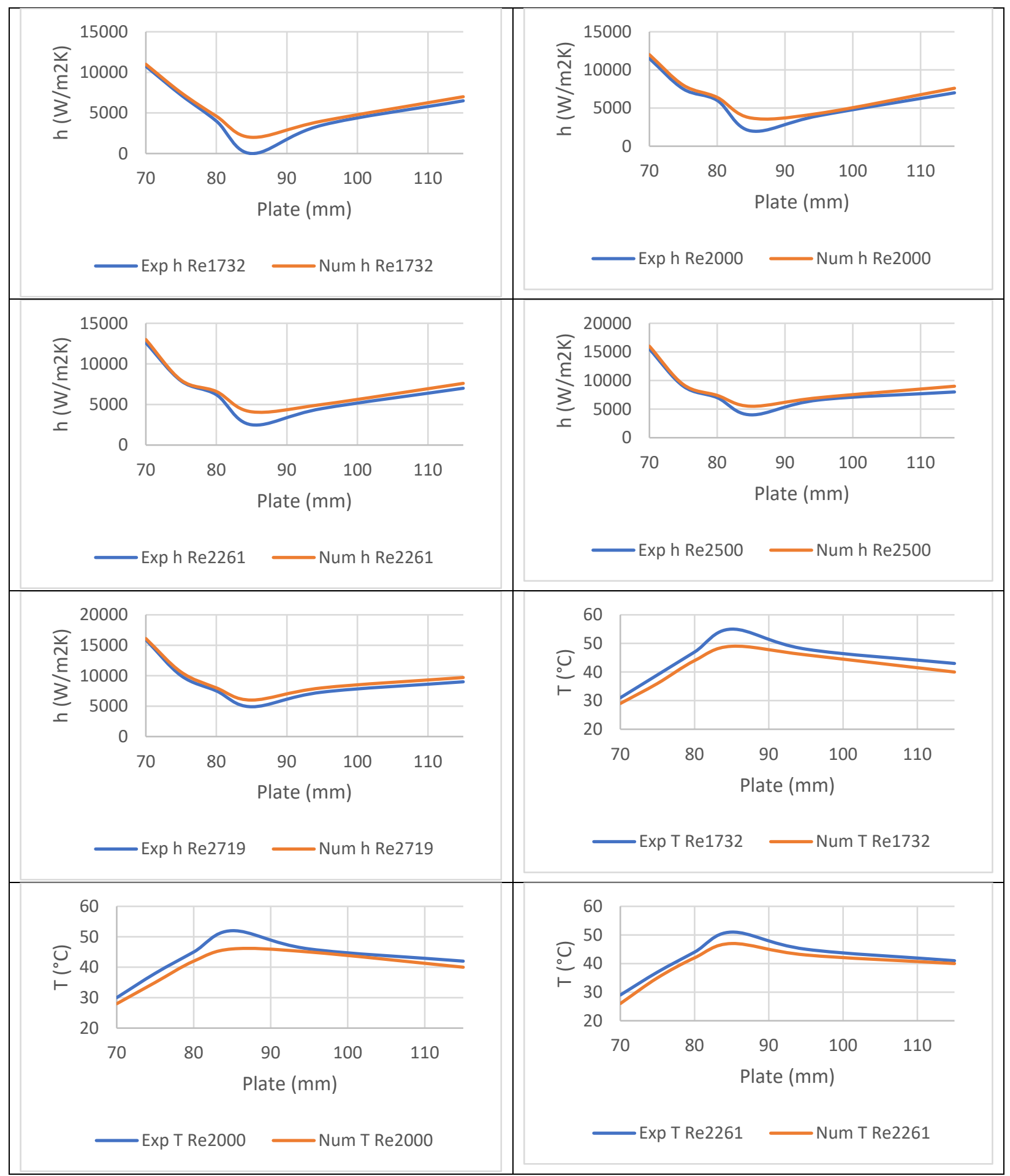




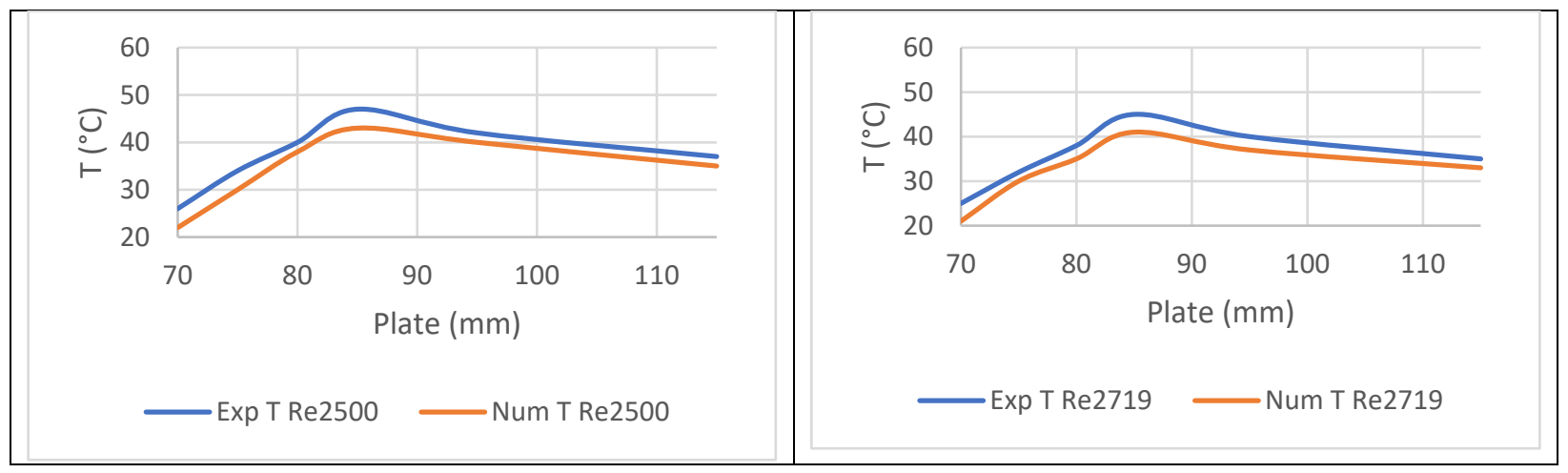

NANOFLUID 0.15\%

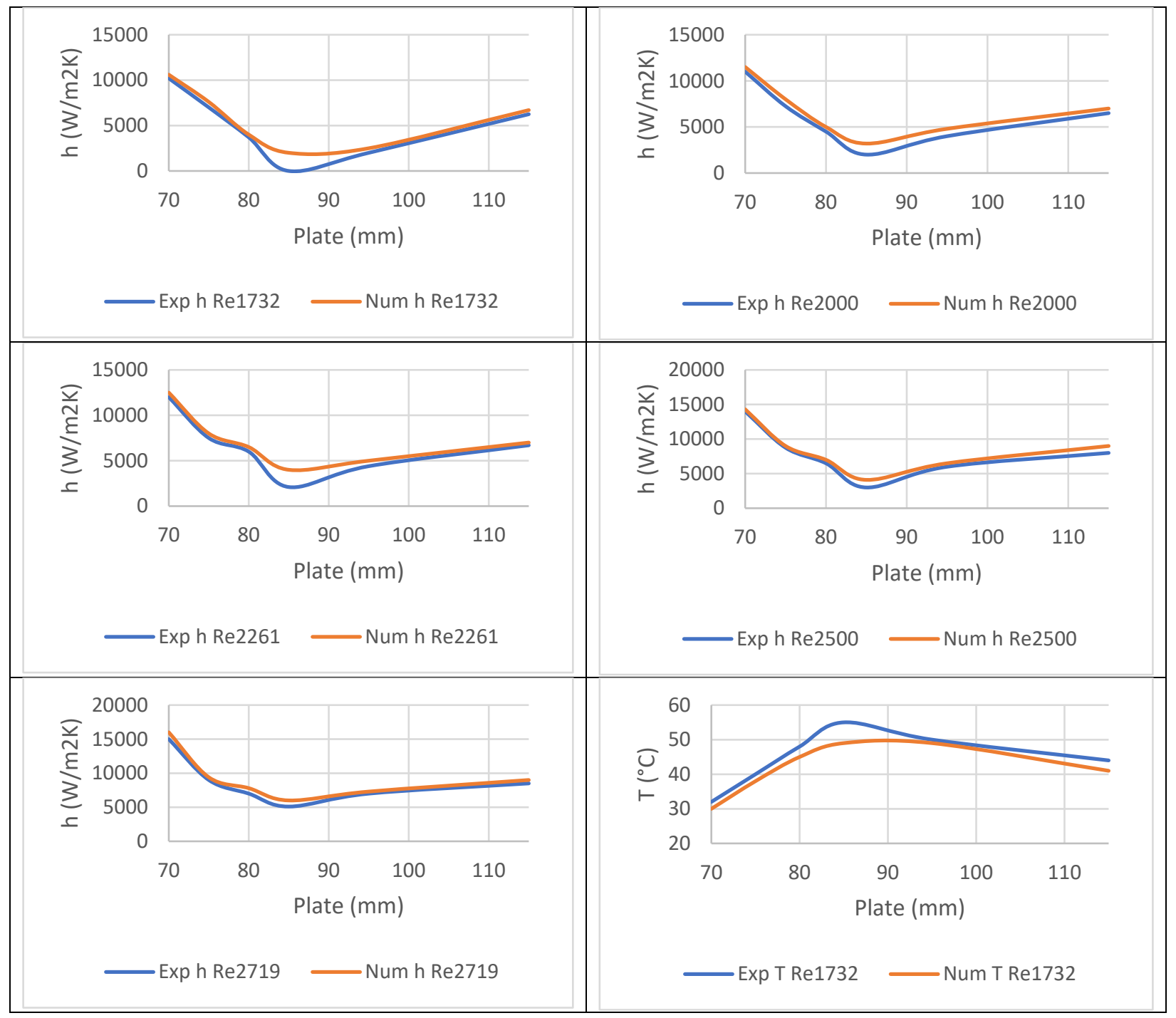




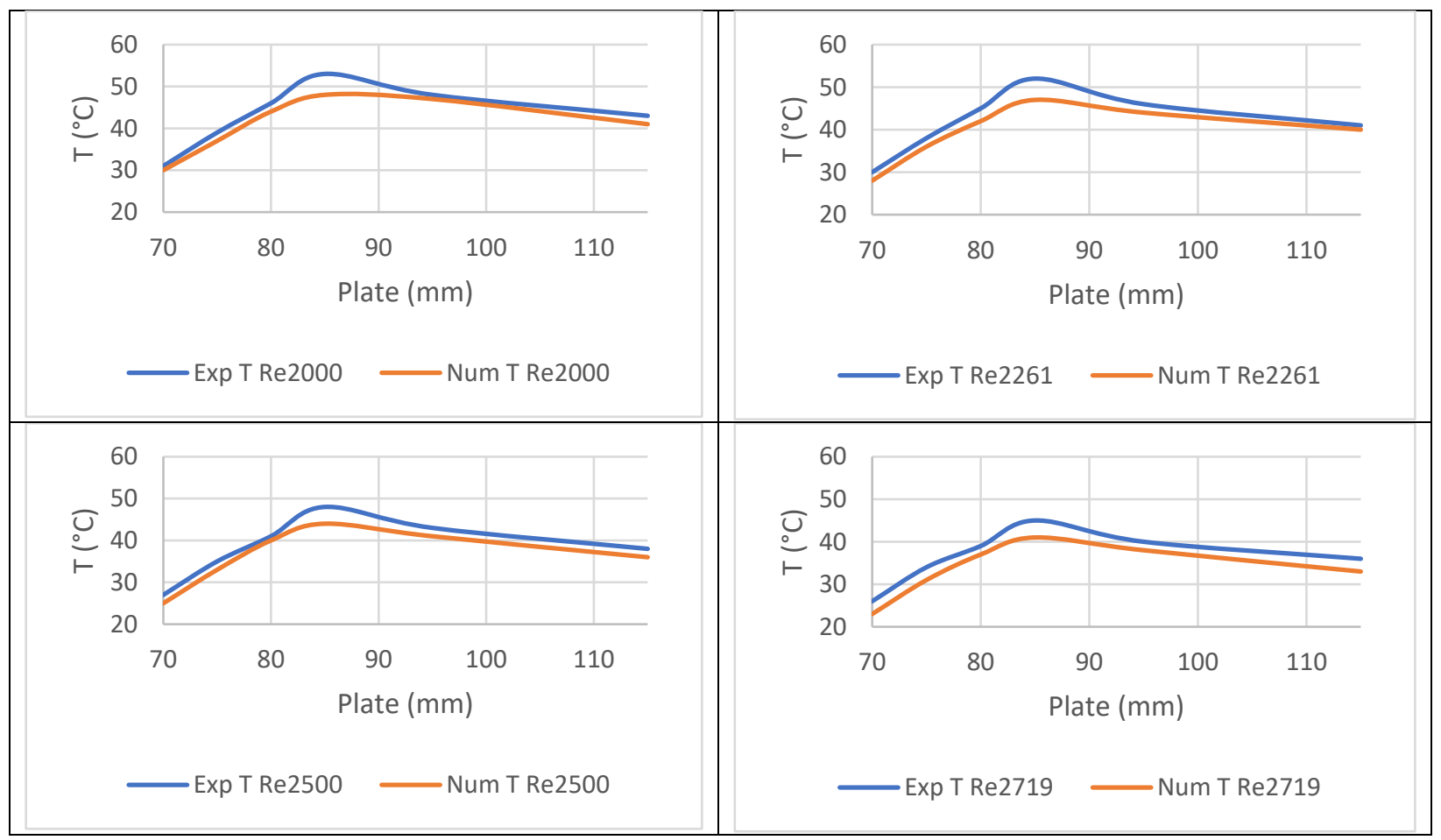




\section{APPENDIX B}

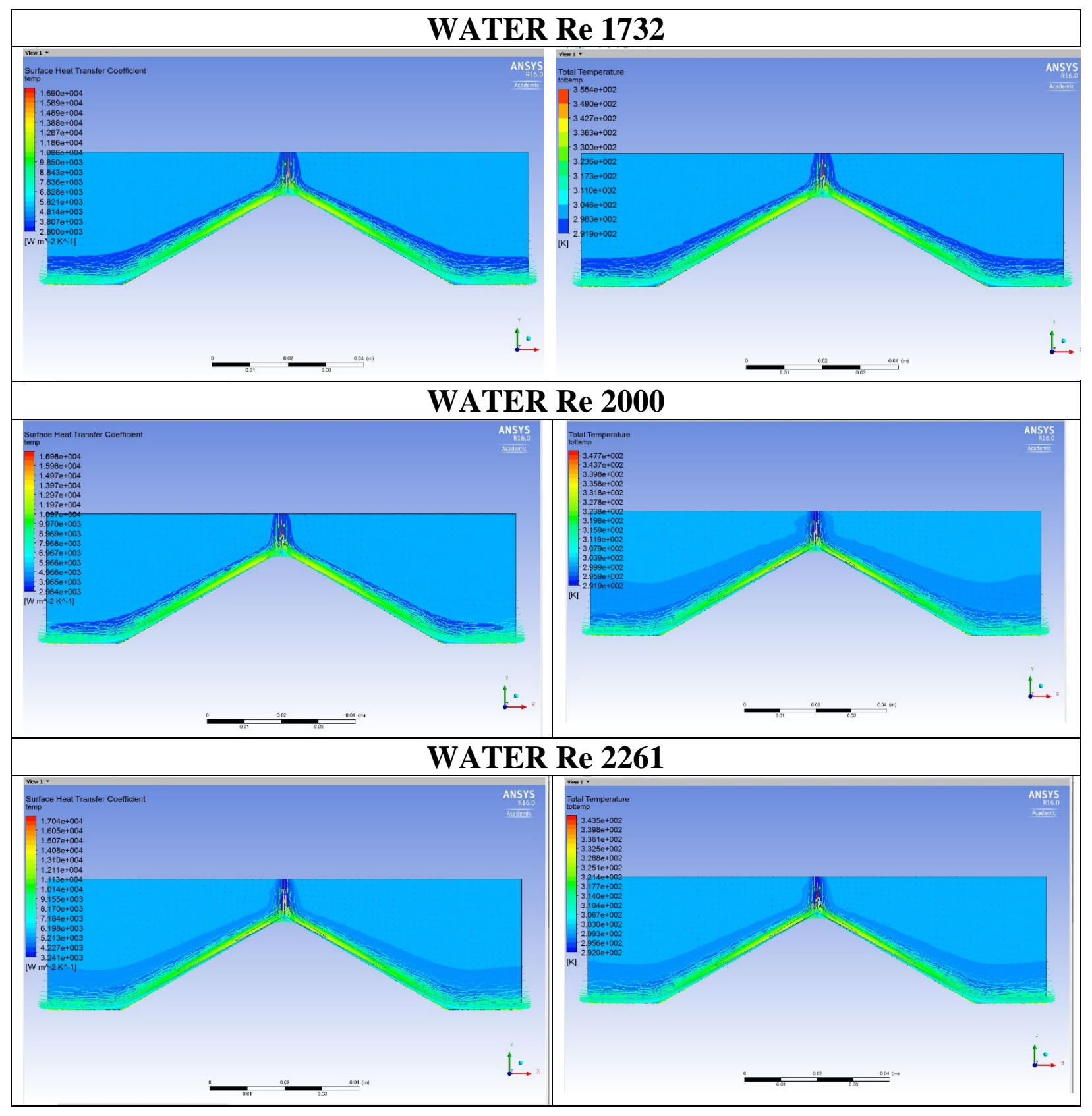




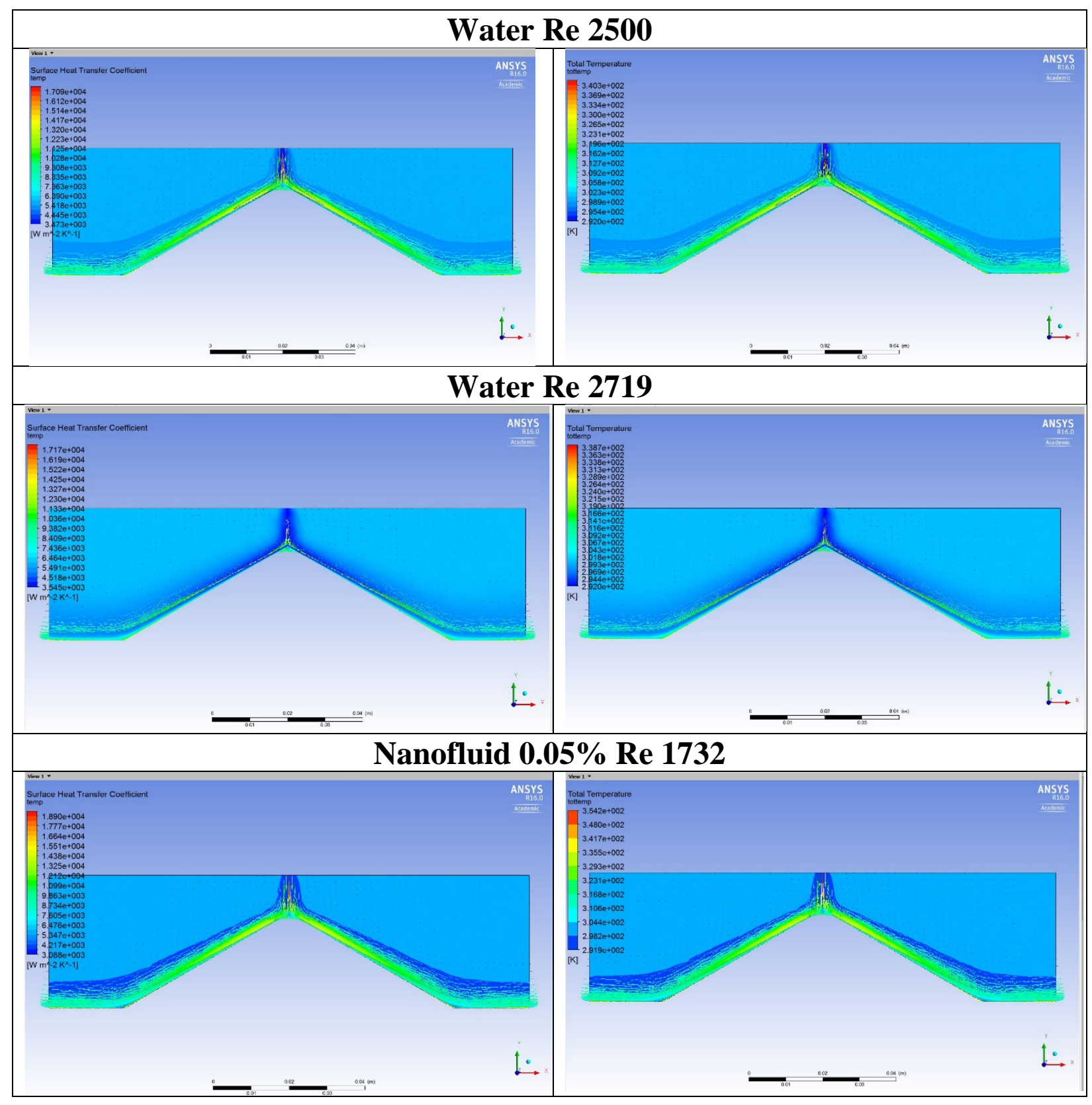




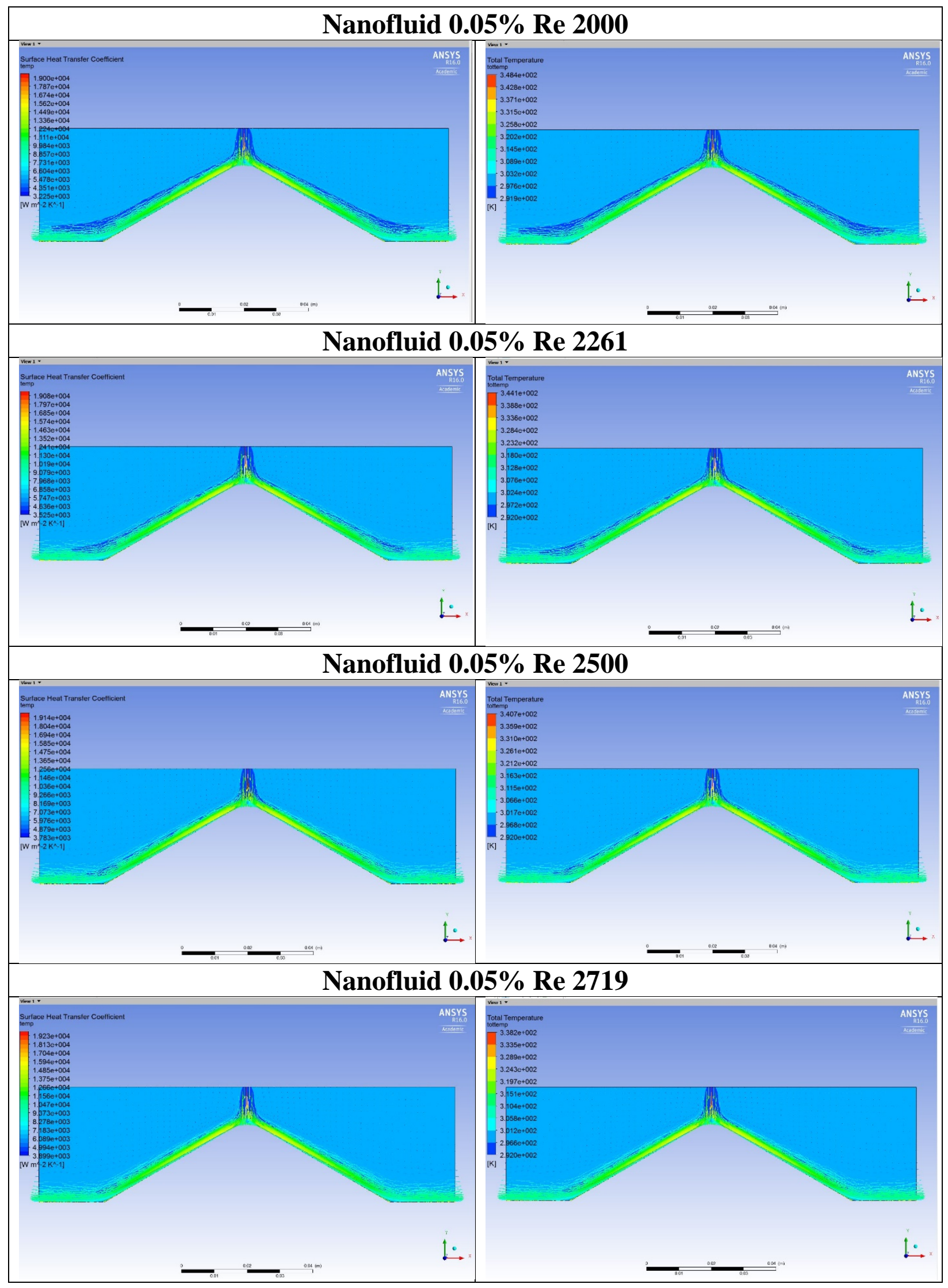




\section{REFERENCES}

[1] X. Liu and J. H. Lienhard, "Exl riments in Fluids The hydraulic jump in circular jet impingement and in other thin liquid films,” Exp. Fluids, vol. 15, pp. 108-116, 1993.

[2] John H. Lienhard, "Liquid Jet Impingement,” Annu. Rev. Heat Transf., vol. 6, 2015.

[3] L. A. Gabour and J. H. Lienhard, "Wall Roughness Effects on StagnationPoint Heat Transfer Beneath an Impinging Liquid Jet,” J. Heat Transfer, 1994.

[4] S. K. Bhunia, "Splattering During Turbulent Liquid Jet Impingement on Solid Targets,” J. Fluids Eng., vol. 116, 1994.

[5] T. Yousefi, B. Jaberi, A. Y. Noohdani, B. Farahbakhsh, and M. Z. Saghir, "Heat transfer enhancement in T-Tube convertor concept using nanofluids," Int. J. Therm. Sci., vol. 91, pp. 125-132, 2015.

[6] X.Liu, "Liquid Jet Impingement Heat Transfer on a Uniform Flux Surface," Heat Transf. Phenom. Radiation, Combusstion, Fires, vol. HTD-106, 1989.

[7] Y. Xuan and W. Roetzel, "Conceptions for heat transfer correlation of nanofluids,” Int. J. Heat Mass Transf., vol. 43, no. 19, pp. 3701-3707, 2000.

[8] E. E. Michaelides, “Transport properties of nanofluids. A critical review,” J. Non-Equilibrium Thermodyn., vol. 38, no. 1, pp. 1-79, Jan. 2013.

[9] T. Yousefi, E. Shojaeizadeh, H. R. Mirbagheri, B. Farahbaksh, and M. Z. Saghir, "An experimental investigation on the impingement of a planar jet of Al2O3-water nanofluid on a V-shaped plate,” Exp. Therm. Fluid Sci., vol. 50, pp. 114-126, 2013.

[10] U. Rea, T. McKrell, L. wen Hu, and J. Buongiorno, "Laminar convective heat transfer and viscous pressure loss of alumina-water and zirconia-water nanofluids,” Int. J. Heat Mass Transf., vol. 52, no. 7-8, pp. 2042-2048, 2009.

[11] K. S. Hwang, S. P. Jang, and S. U. S. Choi, "Flow and convective heat transfer characteristics of water-based $\mathrm{Al} 2 \mathrm{O} 3$ nanofluids in fully developed laminar flow regime,” Int. J. Heat Mass Transf., vol. 52, no. 1-2, pp. 193199, 2009. 
[12] K. Luo, N. Gui, J. Fan, and K. Cen, "Direct numerical simulation of a twophase three-dimensional planar jet," Int. J. Heat Mass Transf., vol. 64, pp. 155-161, 2013.

[13] Y.-T. Yang and F.-H. Lai, "Numerical study of heat transfer enhancement with the use of nanofluids in radial flow cooling system," Int. J. Heat Mass Transf., vol. 53, no. 25-26, pp. 5895-5904, 2010.

[14] S. E. B. Maïga, S. J. Palm, C. T. Nguyen, G. Roy, and N. Galanis, "Heat transfer enhancement by using nanofluids in forced convection flows," Int. J. Heat Fluid Flow, vol. 26, no. 4, pp. 530-546, Aug. 2005.

[15] A. V. Kuznetsov and D. A. Nield, "Natural convective boundary-layer flow of a nanofluid past a vertical plate," Int. J. Therm. Sci., vol. 49, no. 2, pp. 243-247, Feb. 2010.

[16] S. U. S. Choi and J. A. Eastman, "JAM 111935 bTI ENHANCING THERMAL CONDUCTIVITY OF FLUIDS WITH NANOPARTICLES*,” 1995.

[17] S. Lee, S. U.-S. Choi, S. Li, and J. A. Eastman, "Measuring Thermal Conductivity of Fluids Containing Oxide Nanoparticles," J. Heat Transfer, vol. 121, no. 2, p. 280, 1999.

[18] R. L. Hamilton and O. K. Crosser, "Thermal Conductivity of Heterogeneous Two-Component Systems,” Ind. Eng. Chem. Fundam., vol. 1, no. 3, pp. 187191, Aug. 1962.

[19] H. Masuda, A. Ebata, K. Teramae, and N. Hishinuma, “Alteration of Thermal Conductivity and Viscosity of Liquid by Dispersing Ultra-Fine Particles. Dispersion of Al2O3, SiO2 and TiO2 Ultra-Fine Particles.," Netsu Bussei, vol. 7, no. 4, pp. 227-233, 1993.

[20] S. P. Jang and S. U. S. Choi, "Role of Brownian motion in the enhanced thermal conductivity of nanofluids," Appl. Phys. Lett., vol. 84, no. 21, pp. 4316-4318, May 2004.

[21] G. K. Batchelor, "The effect of Brownian motion on the bulk stress in a suspension of spherical particles,” J. Fluid Mech., vol. 83, no. 1, p. 97, Nov. 1977.

[22] B. C. Pak and Y. I. Cho, "HYDRODYNAMIC AND HEAT TRANSFER STUDY OF DISPERSED FLUIDS WITH SUBMICRON METALLIC OXIDE PARTICLES,” Exp. Heat Transf., vol. 11, no. 2, pp. 151-170, Apr. 1998. 
[23] F. W. Dittus, Heat transfer in automobile radiators of the tubular type,. Berkeley, Calif.: University of California press, 1930.

[24] B. C. Pak and Y. I. Cho, "HYDRODYNAMIC AND HEAT TRANSFER STUDY OF DISPERSED FLUIDS WITH SUBMICRON METALLIC OXIDE PARTICLES,” Exp. Heat Transf., vol. 11, no. 2, pp. 151-170, Apr. 1998.

[25] J. Buongiorno, “Convective Transport in Nanofluids,” J. Heat Transfer, vol. 128, no. 3, p. 240, 2006.

[26] FLUENT theory guide and user manual, ANSYS FLUENT 16.0

[27] Y.A. Cengel and A.J. Ghajar, Heat and Mass transfer Fundamentals \& Applications , Fifth edition, McGraw Hill education, 2014

[28] J.D. Anderson Jr., Computational Fluid Dynamics, the basics with Applications, first edition, McGraw Hill education, 1990

[29] S.K. Das, S.S. Choi, W. Yu, T. Pradeep, Nanofluids science \& Technology, first edition, Wiley Interscience, 2008

[30] P. Abgrall, N.T. Nguyen, Nanofluidics, first edition, Artech House, 2009 